\title{
Analysis of a Model for Foam Improved Oil Recovery
}

\author{
P. Grassia ${ }^{1} \dagger$, E. Mas-Hernández ${ }^{1}$, N. Shokri ${ }^{1}$, \\ S. J. Cox ${ }^{2}$, G. Mishuris ${ }^{2}$ and W. R. Rossen ${ }^{3}$ \\ ${ }^{1}$ CEAS, The Mill, University of Manchester, Oxford Rd, Manchester M13 9PL, UK \\ ${ }^{2}$ DMaP, Aberystwyth University, Aberystwyth, Ceredigion, SY23 3BZ, UK \\ ${ }^{3}$ Dept. of Geotechnology, Delft University of Technology, Stevinweg 1, 2628 CN Delft,
} Netherlands

(Received ?; revised ?; accepted ?. - To be entered by editorial office)

During improved oil recovery, gas may be introduced into a porous reservoir filled with surfactant solution in order to form foam. A model for the evolution of the resulting foam front known as 'pressure-driven growth' is analysed. An asymptotic solution of this model for long times is derived that shows that foam can propagate indefinitely into the reservoir without gravity override. Moreover 'pressure-driven growth' is shown to correspond to a special case of the more general 'viscous froth' model. In particular, it is a singular limit of the viscous froth, corresponding to the elimination of a surface tension term, permitting sharp corners and kinks in the predicted shape of the front. Sharp corners tend to develop from concave regions of the front. The principal solution of interest has a convex front, however, so that although this solution itself has no sharp corners (except for some kinks that develop spuriously owing to errors in a numerical scheme), it is found nevertheless to exhibit milder singularities in front curvature, as the long-time asymptotic analytical solution makes clear. Numerical schemes for the evolving front shape which perform robustly (avoiding the development of spurious kinks) are also developed. Generalisations of this solution to geologically heterogeneous reservoirs should exhibit concavities and/or sharp corner singularities as an inherent part of their evolution: propagation of fronts containing such 'inherent' singularities can be readily incorporated into these numerical schemes.

\section{Key words:}

Complex Fluids: Foams; Low-Reynolds-number Flows: Porous media; Mathematical Foundations: Computational methods.

\section{Introduction}

During oil production from geological formations, only a fraction of the oil tends to be extracted during the so called primary recovery phase, prior to the oil reservoir becoming too depleted to push additional oil out under its own pressure (Lake 2010). Secondary recovery techniques are then employed, which consist of injecting a fluid (usually water) into the reservoir with the aim of maintaining pressure and displacing the oil that is still present (Green \& Willhite 1998). Normally the injected fluid is less viscous (i.e. more mobile) than the oil which it drives out, which can lead to fingering phenomena (Ashoori et al. 2010; Casademunt \& Magdaleno 2000; Cottin et al. 2010a,b; Couder et al. 1986;

$\dagger$ Email address for correspondence: paul.grassia@manchester.ac.uk 
Kessler \& Levine 1986a,b; Moore et al. 2002; Pitts 1980; Saffman \& Taylor 1958; Sahimi 2011; Tabeling et al. 1987; Thome et al. 1989): fingers of the driving fluid penetrate and break through the oil, after which additional injected fluid will continue to follow the already established flow paths, without displacing any more oil. This viscous instability initially follows, and greatly magnifies, heterogeneities in permeability within the reservoir (Lake 2010).

Tertiary or improved recovery techniques use various strategies to increase the amount of oil that can be extracted (over and above what the secondary recovery techniques achieve (Lake 2010)). Several of these techniques involve injection of gas $\left(\mathrm{CO}_{2}\right.$, hydrocarbon gas, steam or $\mathrm{N}_{2}$ ), which face the additional challenge that they are less dense than the oil; they tend to rise to the top of the reservoir and override most of the oil. Foam improved oil recovery (abbreviated here as 'foam IOR') can address the problems of viscous instability, geological heterogeneity and gravity override (Kovscek \& Radke 1994; Rossen 1996; Schramm \& Wassmuth 1994), with gravity segregation (i.e. override) being worst if the reservoir is relatively homogeneous (Shan \& Rossen 2004). Foam is relatively immobile in an oil reservoir due to the forces that are required to drive foam films through the channels in a porous medium (Cox et al. 2004b; Falls et al. 1988; Hirasaki et al. 2011; Kovscek \& Bertin 2003a,b; Ma et al. 2013; Rossen 1990a,b; Xu \& Rossen 2003). This relative immobility of foam helps to suppress the fingering phenomena that would otherwise occur, leading to a more uniform displacement of oil from the reservoir than in secondary recovery techniques. In addition there is a chance to divert foam into regions which may have been left unswept during any earlier recovery (Bertin et al. 1999; Li et al. 2010; Ma et al. 2012; Yan et al. 2006): the mechanism (Bertin et al. 1998) is believed to be that the previously unswept regions (which are typically less permeable than the previously swept ones) exert a greater capillary pressure on foam films (Khatib et al. 1988; Kovscek et al. 1995), making those films more likely to burst, thereby selectively enhancing foam mobility in previously unswept regions. Foam also indirectly helps prevent gravity segregation (Rossen et al. 2010; Shan \& Rossen 2004).

During foam IOR, the foam is created in one of two ways: co-injection of gas and surfactant solution, or so called SAG (surfactant alternating gas) where foam is produced in situ by injecting alternately slugs of aqueous surfactant solution and slugs of gas (Afsharpoor et al. 2010; Blaker et al. 2002; Hirasaki et al. 2011; Hoefner \& Evans 1995; Kam 2008; Rossen \& Bruining 2007; Xu \& Rossen 2004; Zanganeh \& Rossen 2013). SAG is usually favoured for field application for operational reasons (Shan \& Rossen 2004). Moreover, if gravity segregation is the primary problem, co-injection processes suffer from excessive pressure dissipation in the near-wellbore region; to maintain injection rate sufficient to overcome gravity segregation one risks fracturing the formation (Rossen et al. 2010). SAG processes allow injection at much lower pressure, while maintaining low mobility at the front of the foam bank. In particular, the limiting case of a SAG process with one large slug of surfactant solution injected ahead of one large slug of gas is remarkably effective at preventing gravity override of gas (Shan \& Rossen 2004).

Before considering how the foam interacts with the oil phase, it is first necessary to consider how the gas and surfactant solution interact to form and subsequently displace foam. This is the particular process we shall consider here. We also consider a geologically homogeneous reservoir, the worst case for gravity segregation (Shan \& Rossen 2004). Gas-surfactant interactions can be described by a theory known as fractional flow theory (Ashoori et al. 2010; Lake 2010; Rossen et al. 1999; Zhou \& Rossen 1995), an application of the method of characteristics. This theory admits a shock that migrates through the reservoir (Shan \& Rossen 2004; de Velde Harsenhorst et al. 2014) separating a continuous aqueous region (downstream of the shock) and a gas rich foamy region 
(upstream of the shock). The foam dries out further and further upstream of the shock, eventually becoming sufficiently dry that the foam starts to collapse.

The result is that gas mobility is large near the injection well but small behind the shock, which is the ideal condition for overcoming gravity override. Figure 1 shows an example. Laboratory corefloods confirm the general shape of the profile in Figure 1 (Ma et al. 2013), even though it is not clear that the foam is fully at local equilibrium, as assumed in fractional-flow theory, in laboratory-scale experiments. Moreover, fractionalflow theory proved accurate and provided key insights into a SAG foam field trial in the Snorre field (Martinsen \& Vassenden 1999).

That the lowest mobilities in the system tend to correspond to a zone of wet foam immediately upstream of the shock may seem at first sight paradoxical, since in traditional foam rheology (outside porous media), dry foams are less mobile than wet ones (Kraynik 1988; Weaire \& Hutzler 1999). In this case however, the dry foam becomes mobile because films collapse as the foam dries out, meaning the dry foam has relatively few bubbles and hence relatively few films to restrict its mobility.

The low mobility, wet foam zone spreads over larger and larger distances as the shock propagates - although its thickness is somewhat smaller than the distance over which the shock itself has moved. This prompted Shan \& Rossen (2004) and de Velde Harsenhorst et al. (2014) to study an idealised model (Figure 2(a)) in which:

- the reservoir is geologically homogeneous,

- only two phases are flowing: gas and water. Immobile, residual oil may be present; such would be the case with a miscible displacement of oil by gas at the leading edge of the foam bank (cf. de Velde Harsenhorst et al. (2014)),

- enough surfactant solution has been injected ahead of the gas that wherever the gas travels it encounters water with surfactant,

- the aqueous region (downstream) and dry foam region (upstream) are assumed to be perfectly mobile compared to the wet foam zone near the shock (see Figure 1), and

- the thickness of the wet foam zone is much less than the distance through which the shock has moved: the combined shock and wet foam zone can then be modelled as a curve (of negligible thickness) propagating through the reservoir over time.

Shan \& Rossen (2004) used this model to present some preliminary numerical results for foam propagation into a reservoir. However they gave little consideration to the underlying mathematical structure of their model, and how it might impact on the choice of numerical scheme: without due care, numerical schemes are known to encounter stability issues for this particular model as de Velde Harsenhorst et al. (2014) demonstrated. In addition to numerical results, de Velde Harsenhorst et al. (2014) also obtained an analytical solution, which is valid provided, during their entire displacement history to date, individual points within the wet foam zone have not moved any significant vertical distance through the system: that is, in the language of petroleum engineering, if vertical permeability is zero. If, on the other hand, the reservoir is assumed isotropic, i.e. equally permeable in horizontal and vertical directions, the numerical results show that points in the wet foam zone actually migrate vertically fairly rapidly. In that case, the analytical solution of de Velde Harsenhorst et al. (2014) is valid only for very early times.

Despite the simplicity of the model, it achieved a number of practical results:

- It showed the remarkable ability of foam, in principle, to overcome gravity override over extremely long horizontal distances, for instance several $\mathrm{km}$ in the case studied by de Velde Harsenhorst et al. (2014), a conjecture verified by subsequent numerical simulation with a conventional foam simulator. The shape of the foam front matched predictions of the $2 \mathrm{D}$ field-scale simulation well.

- It shows that in such a process, it is reservoir extent in the vertical direction, pres- 
sure difference between wells and density difference between phases that control gravity override, not the details of the foam formation and/or collapse process (Shan \& Rossen 2004). Simulations showed that one foam 100 times weaker than another (i.e. differing by a factor of 100 in the amount foam reduces gas mobility relative to the foam-free state) still achieved roughly comparable results given the same well-to-well pressure difference.

- It explains how a numerical artifact (spurious exceptionally low mobility in one grid block at the shock in Figure 1; see Rossen (2013)) can give the false result of effective control of gravity override in a case where the foam breakdown model predicts complete foam collapse immediately behind the shock (Shan \& Rossen 2004). In reality, gravity control of override relies on the foam breakdown model preventing collapse until a small (but finite) distance behind the shock.

- The parameters can be fit (de Velde Harsenhorst et al. 2014) to steady-state laboratory foam-mobility data, and the injection rate needed to maintain a process of fixed injection pressure in a given five-spot well pattern can be estimated directly from the so called fractional-flow curve (Boeije \& Rossen 2014).

Except for the case of zero vertical permeability, de Velde Harsenhorst et al. (2014) encountered severe numerical problems solving their model equations and present only partial results. The numerical problems grew with the time duration of the displacement.

Our objective here is to revisit the model of Shan \& Rossen (2004) and de Velde Harsenhorst et al. (2014), with a particular focus on the mathematical structure of the governing equations and the underlying physical implications. We give consideration both to longtime asymptotic solutions and to selection of suitable numerical schemes for finite time. Remarkably, we find that the model of Shan \& Rossen (2004) designed to apply to a foam front comprising innumerable tiny bubbles on a scale of hundreds of metres, maps as a special case of a so called 'viscous froth model' (Glazier \& Weaire 1992; Weaire \& McMurry 1996), derived for experiments on individual soap films between glass plates on a scale of $\mathrm{cm}$ or $\mathrm{mm}$ (Drenckhan et al. 2005). With our approach, we derive asymptotic solutions for long times that are free of numerical instabilities; this asymptotic form fits the numerical results of de Velde Harsenhorst et al. (2014) for relatively long times well. Because this asymptotic form applies in the limit of long times, it means there is in principle no limit to how far foam could travel without gravity segregation. Thus this work both confirms and extends the partial results of de Velde Harsenhorst et al. (2014). We identify and explore the causes of the numerical instabilities seen by de Velde Harsenhorst et al. (2014), and discuss how these instabilities are inherent to the dynamics if the model is extended to heterogeneous reservoirs. We present better ways to interpolate points in the numerical solution for the front and to control numerical instabilities than those proposed by de Velde Harsenhorst et al. (2014).

It was mentioned earlier that one of the rationales for using foam IOR is to access parts of a reservoir which may have been left unswept by previous recovery techniques. Although foam helps to prevent gravity override, even foam IOR will still not give a perfectly efficient (i.e. perfectly plug-like) reservoir sweep. Instead, if foam enters an injection well and subsequently arrives at a production well, there is likely to be a region located deep below the production well which remains unswept by foam. The more uniform the displacement of the foam front with depth, the smaller this unswept region becomes. Thus knowledge of the shape of the foam front is very relevant to petroleum engineers wanting to understand which parts of a reservoir might remain unswept at any given instant. Indeed this is one of the main engineering questions to be addressed here.

The rest of this paper is laid out as follows. In Section 2 we briefly derive the model of Shan \& Rossen (2004) and de Velde Harsenhorst et al. (2014). In Section 3 we analyse the mathematical structure of the model - and show that it can be regarded as a 
special case of a more general model, the viscous froth (Glazier \& Weaire 1992; Weaire \& McMurry 1996) that has been already well-studied in the foams literature: the Shan \& Rossen (2004)/de Velde Harsenhorst et al. (2014) model is shown to be a singular limit of that more general model, so it actually admits some rather special behaviour. Asymptotic analysis (Section 4) and numerics (Section 5) then follow, where the special and singular nature of the model again impacts on the results. Section 6 contains a general discussion with the outlook for future use of the model: in particular Section 6 mentions a number of unsolved problems of relevance to foam IOR where the Shan \& Rossen (2004)/de Velde Harsenhorst et al. (2014) model is likely to present interesting physical behaviour. Conclusions are offered in Section 7 .

\section{Model derivation}

In this section the model of Shan \& Rossen (2004) and de Velde Harsenhorst et al. (2014) is derived. Readers already familiar with this derivation may wish to turn directly to Section 3 where the formal mathematical structure of the model is considered.

A definition sketch for the system under consideration is shown in Figure 2. For simplicity, the sketch envisages a 2-dimensional model geometry. Shan \& Rossen (2004) considered both a 2-dimensional model, and axisymmetric flow about an injection well. The predicted shapes of the advancing foam front turn out to be the same in either case, but the volume of material injected to achieve a given front shape is geometry dependent.

The model begins by considering Darcy's law for gas in the aforementioned 'low mobility' zone, which states

$$
\boldsymbol{u}_{\text {sup }}=-k \lambda_{r} \nabla P
$$

where $\boldsymbol{u}_{\text {sup }}$ is the gas superficial velocity, $k$ is the reservoir permeability, $\lambda_{r}$ is the so called 'gas relative mobility' (the reciprocal of an effective viscosity), and $\nabla P$ is a driving pressure gradient.

The low mobility zone itself translates at the interstitial velocity $\boldsymbol{u}_{\text {int }}$ rather than the superficial velocity $\boldsymbol{u}_{\text {sup }}$. If $\boldsymbol{x}$ denotes the position of an element within the low mobility zone, and $t$ denotes time, then the rate of advance $\mathrm{d} \boldsymbol{x} / \mathrm{d} t$ satisfies

$$
\frac{\mathrm{d} \boldsymbol{x}}{\mathrm{d} t}=\boldsymbol{u}_{\text {int }}=-\frac{k \lambda_{r}}{\left(1-S_{w}\right) \phi} \nabla P
$$

where $S_{w}$ is the volume fraction of water in the foam (see note $(a)$ in the 'notes on the text'), and $\phi$ is the porosity of the medium.

The pressure gradient across the low mobility zone is

$$
\nabla P=-\frac{\Delta P}{\tau_{\text {thick }}} \boldsymbol{n}
$$

where $\Delta P$ is the pressure difference across the low mobility zone (from the foam region into the aqueous region), $\tau_{\text {thick }}$ is the thickness of the low mobility zone (which grows with the distance the front has displaced), and $\boldsymbol{n}$ is the unit normal to the front (pointing into the aqueous region).

It follows that

$$
\frac{\mathrm{d} \boldsymbol{x}}{\mathrm{d} t}=\frac{k \lambda_{r}}{\left(1-S_{w}\right) \phi} \frac{\Delta P}{\tau_{\text {thick }}} \boldsymbol{n} .
$$

Based on fractional flow theory (Shan \& Rossen 2004), the thickness of the low mobility zone is assumed to obey

$$
\tau_{\text {thick }}=\tau s
$$


where $s$ is the distance through which a particular element of front has displaced, and $\tau$ is a constant (assumed to be considerably smaller than unity).

Additionally in the above

$$
\Delta P=P_{\text {drive }}-P_{\text {hyd }}
$$

where $P_{\text {drive }}$ is a driving pressure for the injection (treated as uniform), $P_{\text {hyd }}$ is the hydrostatic pressure difference between the upstream gas (in the foam bank) and the downstream liquid in the aqueous phase: $P_{h y d}$ increases with depth. Following de Velde Harsenhorst et al. (2014), we assume the hydrostatic pressure difference $P_{\text {hyd }}$ to be

$$
P_{h y d}=\rho g\left(d_{\max }-y\right)
$$

where $\rho$ is the density difference between the aqueous region and the gas in the foam bank, $g$ is gravity, $d_{\max }$ is the maximum depth to which foam can penetrate, and $y$ is a coordinate measured upwards, such that $y=0$ at the point of maximum depth, and $y=d_{\max }$ at the surface. It follows therefore that

$$
d_{\max }=\frac{P_{\text {drive }}}{\rho g} .
$$

We make the above system of equations dimensionless by scaling lengths (and thicknesses) by $d_{\max }$, pressures by $P_{\text {drive }}$, and scaling times by a quantity $t_{\text {scale }}$ defined as

$$
t_{\text {scale }}=\frac{\left(1-S_{w}\right) \phi}{k \lambda_{r}} \frac{d_{\max }^{2}}{P_{\text {drive }}} \tau .
$$

The only parameter in the model that depends on the details of foam behaviour is the ratio $\lambda_{r} / \tau$. Viscous and less-viscous foams correspond to small and large values of $\lambda_{r} / \tau$, respectively (Boeije \& Rossen 2014). As has been shown by Shan \& Rossen (2004) (utilising data from Fisher et al. (1990) and Persoff et al. (1991)), the shape of the foam front is nearly independent of the viscosity of the foam front (i.e. of $\lambda_{r} / \tau$ ), but the physical time required to inject a given volume of foam does depend on $\lambda_{r} / \tau$.

Using primes to denote dimensionless variables it follows

$$
\frac{\mathrm{d} \boldsymbol{x}^{\prime}}{\mathrm{d} t^{\prime}}=\frac{\Delta P^{\prime}}{s^{\prime}} \boldsymbol{n}
$$

where

$$
\Delta P^{\prime}=y^{\prime}
$$

with also

$$
\tau_{\text {thick }}^{\prime}=\tau s^{\prime} .
$$

The evolution of $s^{\prime}$ can be deduced from the dot product of equation (2.10) with the normal vector $\boldsymbol{n}$ :

$$
\frac{\mathrm{d} s^{\prime}}{\mathrm{d} t^{\prime}}=\frac{\Delta P^{\prime}}{s^{\prime}} .
$$

Typically we think of the case $\tau_{\text {thick }}^{\prime} \ll s^{\prime}$, i.e. the model applies to an idealised situation where the thickness of the wet foam zone near the front is much less than the distance through which the front itself displaces. It is then meaningful to represent the foam front at any instant as a 1-dimensional curve (as Figure 2(b) indicates).

The above equations need to be solved with suitable boundary and initial conditions. Shan \& Rossen (2004) assumed a boundary condition at the top of the foam front that elements move purely horizontally, i.e.

$$
\boldsymbol{n} \cdot \boldsymbol{e}_{x}=1
$$


where $\boldsymbol{e}_{x}$ is the Cartesian unit vector.

This boundary condition can be expressed in various equivalent ways. For example, Shan \& Rossen (2004) and de Velde Harsenhorst et al. (2014) defined an angle $\alpha$ (see the sketch in Figure 2(b) for the definition of $\alpha$ )

$$
\alpha=\arccos \left(\boldsymbol{n} . \boldsymbol{e}_{x}\right)
$$

so the boundary condition at the top implies $\alpha=0$. Here for convenience we also define $\alpha_{c}$ to be the complement to angle $\alpha$

$$
\alpha_{c}=\arccos \left(-\boldsymbol{n} \cdot \boldsymbol{e}_{y}\right)
$$

where again $\boldsymbol{e}_{y}$ is a Cartesian unit vector, and which requires that $\alpha_{c}=\frac{\pi}{2}$ at the top boundary. Moreover, since the top of the front moves horizontally, we have $y^{\prime}=1$ there and $x^{\prime}=s^{\prime}$ and hence via equations (2.11) and (2.13), our boundary condition implies

$$
x^{\prime}=s^{\prime}=\sqrt{2 t^{\prime}+s_{0}^{\prime 2}} \text { at } y^{\prime}=1
$$

for some given value $s_{0}^{\prime}$ (to be identified shortly). Although we treat equation (2.17) as deriving from conditions (2.14)-(2.16), it is also possible to treat equation (2.17) as our boundary condition, and then to deduce the top boundary values of $\boldsymbol{n}=\boldsymbol{e}_{x}, \alpha=0$ and $\alpha_{c}=\frac{\pi}{2}$ as consequences of it. This subtle distinction between the two ways of formulating the boundary condition, will become pertinent much later on (in Section 5.1).

The initial condition meanwhile is that (for any $y^{\prime}$ in the domain $0 \leqslant y^{\prime} \leqslant 1$ ),

$$
x^{\prime}=s^{\prime}=s_{0}^{\prime} \text { at } t^{\prime}=0
$$

again for some particular value $s_{0}^{\prime}$. We do not choose $s_{0}^{\prime} \rightarrow 0$, since this would give infinite velocity $\mathrm{d} \boldsymbol{x}^{\prime} / \mathrm{d} t^{\prime} \rightarrow \infty$. In reality the foam front can never propagate infinitely fast - since even when the low mobility wet foam zone at the front is itself extremely narrow - the aqueous region (downstream) and dry foam region (upstream) still have finite (albeit high) mobilities. Consulting data reported in Shan \& Rossen (2004), these high mobility regions might be at least two orders of magnitude more mobile than the wet foam region at the front, suggesting that a suitable choice for $s_{0}^{\prime}$ on the order of

$$
s_{0}^{\prime} \sim 10^{-2} .
$$

As time proceeds, the solutions become insensitive to the exact value chosen for $s_{0}^{\prime}$, but choosing a finite $s_{0}^{\prime}$ is important to keep the solutions regular as $t^{\prime} \rightarrow 0$.

At any subsequent time $t^{\prime}$, once the formula for the foam front $\boldsymbol{x}^{\prime}$ vs $t^{\prime}$ is known, or equivalently once $y^{\prime}$ as a function of $x^{\prime}$ at the given time $t^{\prime}$ is known, the area which has been swept by foam is given by $\int_{0}^{x_{t o p}}\left(1-y^{\prime}\right) \mathrm{d} x^{\prime}$, where $x_{t o p}$ denotes the (dimensionless) position of the leading edge at the top of the foam front. Meanwhile the area of the region which has remained unswept by foam beneath the front is $\int_{0}^{x_{\text {top }}} y^{\prime} \mathrm{d} x^{\prime}$. These are of course 2-dimensional formulae: analogues for the case of axisymmetric flow about an injection well are straightforward to obtain (see note $(b)$ in the 'notes on the text').

This completes our derivation of the model developed by Shan \& Rossen (2004) and de Velde Harsenhorst et al. (2014). In the section to follow we analyse the mathematical structure of the model, and the physical implications thereof.

\section{Mathematical structure of the model}

Equation (2.4) or its dimensionless form (equation (2.10)) constitute a model for what could be termed 'pressure-driven growth'. This can be viewed as a special case of a model 
that is already well-studied in the literature, called the 'viscous froth model' (Glazier \& Weaire 1992; Weaire \& McMurry 1996). The viscous froth model has been studied extensively over the past decade as a promising candidate model for foam rheology (Barry et al. 2010; Cox \& Mishuris 2009; Cox et al. 2004a; Cox 2005; Cox et al. 2009; Drenckhan et al. 2005; Embley \& Grassia 2011; Grassia et al. 2008a; Green et al. 2006, 2009; Kern et al. 2004). The analogy between viscous froth and the 'pressure-driven growth' of Shan \& Rossen (2004)/de Velde Harsenhorst et al. (2014) holds despite the fact that the former model is normally employed to describe the dynamics of an element of a single foam film advancing in the region between two closely-spaced plates, whereas the latter model was employed to describe the motion of an element of an advancing foam front containing a multitude of bubbles and films.

The remainder of this section is laid out as follows. First (in Section 3.1) we make the analogy between 'pressure-driven growth' and the viscous froth model precise. Then in Sections 3.2-3.3 we describe two other special cases of viscous froth, 'curvature-driven growth' and the 'quasi-static soap froth'. These latter two cases are well known in the foams literature (see e.g. Brakke (1978); Fullman (1952); Mullins (1956); Weaire \& Kermode $(1983,1984)$ ), and the connections between them are widely recognised (Smith 1952). The case of 'pressure-driven growth' is much less studied, at least in the context of foams. The reason (as is explained in Sections 3.4-3.5) is that 'pressure-driven growth' is a singular limit of viscous froth where a stabilizing diffusive term has been discarded, whereas the other two special cases ('curvature-driven growth' and the 'quasi-static soap froth') retain that diffusive term and so are non-singular. Owing to the singular limit involved in 'pressure-driven growth', it admits solutions (see Section 3.6) that are much less regular than those from either viscous froth or its non-singular special cases. Indeed 'pressure-driven growth' turns out to be equivalent to the so called 'eikonal equation' (Arnold 2004), which is known to allow singular solutions, making it particularly challenging to solve. It is anticipated that some readers may already be well acquainted with the viscous froth model and its various limiting cases: such readers may wish to turn directly to Section 4 which deals specifically with solutions for 'pressure-driven growth'.

\subsection{Mathematical analogy with the viscous froth model}

We now make the analogy between viscous froth and pressure-driven growth precise. In dimensional form the viscous froth model can be written (see also note $(c)$ ):

$$
\frac{\mathrm{d} \boldsymbol{x}}{\mathrm{d} t}=\frac{k \lambda_{r}}{\left(1-S_{w}\right) \phi} \frac{(\Delta P-\gamma \kappa)}{\tau_{\text {thick }}} \boldsymbol{n}
$$

where $\gamma$ denotes a surface tension, and $\kappa$ denotes a curvature defined as

$$
\kappa=-\frac{\mathrm{d}^{2} \boldsymbol{x}}{\mathrm{d} S^{2}} \cdot \boldsymbol{n}
$$

with $S$ denoting the arc length measured along the front (again see the definition sketch Figure 2(b)). Note that $S$ is quite distinct from $s$, which is length measured along the paths travelled by material points over time.

The dimensionless analogue of the equation (3.1), which we shall employ in the discussion that follows, is

$$
\frac{\mathrm{d} \boldsymbol{x}^{\prime}}{\mathrm{d} t^{\prime}}=\frac{\left(\Delta P^{\prime}-\gamma^{\prime} \kappa^{\prime}\right)}{s^{\prime}} \boldsymbol{n}
$$

where $\gamma^{\prime}=\gamma /\left(d_{\max } P_{\text {drive }}\right)$, and $\kappa^{\prime}=-\mathrm{d}^{2} \boldsymbol{x}^{\prime} / \mathrm{d} S^{\prime 2} . \boldsymbol{n}$ (with $\left.S^{\prime}=S / d_{\max }\right)$.

Clearly the Shan \& Rossen (2004)/de Velde Harsenhorst et al. (2014) pressure-driven growth model (equation (2.10)) corresponds to the $\gamma^{\prime} \rightarrow 0$ limit of the viscous froth 
model (3.3). Before we consider how the limit $\gamma^{\prime} \rightarrow 0$ affects the viscous froth model, it is valuable to compare and contrast with two other special cases of the viscous froth equation, one being the case of curvature-driven growth (Brakke 1978; Cox 2005; Green et al. 2009; Mullins 1956; Peleg et al. 2001; Weaire \& McMurry 1996) (well studied in the grain growth literature (Fullman 1952; Smith 1952)) and the other being the (again well-studied case) of a 'conventional' quasi-static soap froth (Cantat et al. 2013; Smith 1952; Weaire \& Hutzler 1999; Weaire \& Kermode 1983, 1984).

\subsection{Curvature-driven growth}

The first special case is the limit $\Delta P^{\prime} \rightarrow 0$, giving

$$
\frac{\mathrm{d} \boldsymbol{x}^{\prime}}{\mathrm{d} t^{\prime}}=-\frac{\gamma^{\prime} \kappa^{\prime}}{s^{\prime}} \boldsymbol{n}=\frac{\gamma^{\prime}}{s^{\prime}} \frac{\mathrm{d}^{2} \boldsymbol{x}^{\prime}}{\mathrm{d} S^{\prime 2}} \cdot \boldsymbol{n} \boldsymbol{n} .
$$

As stated above, this corresponds to a model for curvature-driven growth (see e.g. Brakke (1978); Fullman (1952); Mullins (1956)).

Equation (3.4) takes the form of a diffusion equation (see also note $(d)$ ), with $\gamma^{\prime}$ (or more precisely $\gamma^{\prime} / s^{\prime}$ ) representing diffusivity. The quantity which is diffusing here is front curvature, a notion that Satomi et al. (2013) have made mathematically precise.

We explain equation (3.4) physically as follows. Surface tension represents the energy gain associated with decreasing the length of the front. Fronts which have sharp corners or kinks (see note $(e)$ for further explanation) where the front normal $\boldsymbol{n}$, or analogously the front tangent $\boldsymbol{t}$ defined as

$$
\boldsymbol{t} \equiv \mathrm{d} \boldsymbol{x}^{\prime} / \mathrm{d} S^{\prime},
$$

undergoes a step change, can reduce their overall length by rounding off the corner. Sharp corners correspond to points of infinite curvature - so fronts can be considered to reduce their energy by diffusing or spreading their curvature along their length, and this is the physical content of equation (3.4).

\subsection{The quasi-static soap froth}

The next special case of viscous froth that we consider is the quasi-static soap froth. The left hand side of equation (3.3) is discarded, and a Young-Laplace law is assumed to hold

$$
\kappa^{\prime}=\frac{\Delta P^{\prime}}{\gamma^{\prime}} .
$$

In a conventional description of quasi-static soap froth rheology (Cantat et al. 2013; Weaire \& Hutzler 1999; Weaire \& Kermode 1983, 1984), motion is imposed on a foam sample by displacing its boundaries in a specified way, and curvatures of foam films are then sought that satisfy equation (3.6) subject to constraints on bubble 'volumes' (i.e. areas in 2-D) and on angles at which films are permitted to meet one another. Mathematically this is equivalent to a minimisation of overall surface energy of the foam films, subject to constraints on the bubble volumes and on the locations where films meet the system boundary. The imposed boundary motion is assumed to be sufficiently slow, such that viscous forces (associated with the rates of film motion $\mathrm{d} \boldsymbol{x}^{\prime} / \mathrm{d} t^{\prime}$ ) are much smaller than either pressure forces or surface tension-curvature forces. These latter forces then balance as equation (3.6) suggests.

Conventionally the model is applied to each and every bubble film, so that $\Delta P^{\prime}$ is uniform along the film, implying that curvature $\kappa^{\prime}$ is likewise uniform. Bubble films are then arcs of circles - corresponding to the shortest possible film length (and hence smallest possible film energy - see also note $(f)$ ), subject to some volume constraint. 
Another way of saying the same thing, is that curvature has now had sufficient time to diffuse along the entire length of the film to have reached a (quasi)steady state.

To summarise, two special cases of the viscous froth model, namely curvature-driven growth and the conventional quasi-static soap froth model, have received much attention in the foams literature. Indeed the viscous froth model was originally derived to form a 'bridge' between those two special cases (Glazier \& Weaire 1992; Weaire \& McMurry 1996). However the third special case - the pressure-driven growth model of Shan \& Rossen (2004) and de Velde Harsenhorst et al. (2014) - has received surprisingly little attention, at least from within the foams research community.

\subsection{Viscous froth model in the case of small surface tension}

In order to gain physical insight into pressure-driven growth, it is useful to return to the viscous froth equation (3.3) in the case of a small but finite surface tension $\gamma^{\prime}$. With suitable redefinitions of dimensionless pressure and dimensionless velocity, this limit is the same as the high speed limit of viscous froth already considered by Cox et al. (2009); Grassia et al. (2008a); Green et al. (2006). For the present discussion, it proves more convenient however to retain the particular form given in equation (3.3).

If we consider a shape (as in Figure 3) consisting of two comparatively straight sections of front joined by a sharp curve, then it is clear that the pressure will make the straight sections try to advance. However whether the sharply curved part initially advances or recedes depends on whether the curvature $\kappa^{\prime}$ is greater or less than order $\gamma^{\prime-1}$ (the driving pressure $\Delta P^{\prime}$ being order unity in our dimensionless system). If a front curvature on the order of $\gamma^{\prime-1}$ is to be resolved via a numerical scheme, then it is necessary to grid the front down to a spatial resolution $\delta \boldsymbol{x}_{\text {grid }}^{\prime}$ on the order of $\delta \boldsymbol{x}_{\text {grid }}^{\prime} \sim O\left(\gamma^{\prime}\right)$. Given surface tension implies a diffusion of curvature (viz. the discussion following equation (3.4)) with the diffusivity $D_{\text {curv }}$ likewise being on the order $D_{\text {curv }} \sim O\left(\gamma^{\prime}\right)$ (see note $(g)$ ), we require a temporal resolution $\delta t^{\prime} \sim O\left(\delta \boldsymbol{x}_{\text {grid }}^{\prime 2} / D_{\text {curv }}\right) \sim O\left(\gamma^{\prime}\right)$. Viscous froth simulations are clearly expensive numerically whenever $\gamma^{\prime} \ll 1$.

It is natural to ask therefore whether, if the formal limit $\gamma^{\prime} \rightarrow 0$ is taken in the viscous froth equations (thereby recovering the pressure-driven growth model of Shan \& Rossen (2004) and de Velde Harsenhorst et al. (2014)), it is somehow possible to solve cheaply numerically, without needing to resort to such tiny spatial and time steps.

\subsection{The formal limit of zero surface tension}

Eliminating surface tension altogether represents a significant physical change to the system of equations. There is no longer any energy cost associated with additional front length, so there is no incentive for the system to round off any sharp corners.

Remembering that tangent vectors are defined as $\boldsymbol{t}=\mathrm{d} \boldsymbol{x}^{\prime} / \mathrm{d} S^{\prime}$, and assuming a sign convention such that arc length $S^{\prime}$ along the front increases moving downwards from the top of the reservoir, the normal vector is simply defined by

$$
\boldsymbol{n}=\boldsymbol{e}_{z} \wedge \boldsymbol{t}=\boldsymbol{e}_{z} \wedge \mathrm{d} \boldsymbol{x}^{\prime} / \mathrm{d} S^{\prime}
$$

with $\boldsymbol{e}_{z}$ being a Cartesian unit vector out of the plane, so that the governing equation (2.10) becomes

$$
\frac{\mathrm{d} \boldsymbol{x}^{\prime}}{\mathrm{d} t^{\prime}}=\frac{\Delta P^{\prime}}{s^{\prime}} e_{z} \wedge \frac{\mathrm{d} \boldsymbol{x}^{\prime}}{\mathrm{d} S^{\prime}}
$$

Unlike equation (3.3) (or indeed the yet simpler equation (3.4)), this is no longer of parabolic (i.e. diffusive) type, but instead of hyperbolic (i.e. wave-like) type (Renardy \& Rogers 2004). 
It is well known that solutions of hyperbolic equations admit the possibility of discontinuities or 'shocks' (Grassia et al. 2008b, 2011; Kynch 1952; Lester et al. 2005; Lighthill \& Whitham 1955; Renardy \& Rogers 2004; Rossen \& Bruining 2007; Zanganeh et al. 2011). Indeed we have already had cause to mention shocks earlier (in Section 1) in the context of the so called fractional flow theory (Ashoori et al. 2010; Rossen et al. 1999; Zhou \& Rossen 1995), which admits a jump in liquid fraction (between liquid and foam) across a front advancing through the reservoir. Here however we use the word 'shock' in a somewhat different context. We take the jump in liquid fraction across the advancing front as given, and we focus on the geometric shape of the advancing front, so that a shock (in the present context) corresponds to a discontinuity in the tangent vector measured along the front or a sharp corner in the shape of the front itself. In what follows, we shall utilise the term 'shock' exclusively in this particular context (see note $(h)$ ).

It is possible to show that equation (3.8), and likewise equation (2.10) from which it derives, are formally equivalent to the so called eikonal equation (Arnold 2004; Goldstein 1980). This equation has been widely studied in the mathematics community, with specialist numerical techniques having been developed able to handle shocks and corners which arise (Sethian 1996). Applications of the eikonal equation include geometric optics (Arnold 2004; Goldstein 1980), structure of porous media (Haranczyk \& Sethian 2009, 2010), wave propagation in geophysics (Sethian \& Popovici 1999), and crystal growth (Taylor 1995). Foams however represent a relatively new field of application for the eikonal equation (Saye \& Sethian 2011). In our system, material points on the foam front that propagate through an oil reservoir according to pressure-driven growth can be shown to correspond to characteristic curves of the eikonal equation. Meanwhile shocks or corners in the front shape correspond to different characteristics intersecting one another. We consider such shocks in what follows.

\subsection{Analysis of front shapes and their propensity to forming shocks}

It is relatively easy to argue geometrically which front shapes are liable to produce shocks (via the pressure-driven growth equation (2.10)) and which are not. The result turns out to be that shapes which are convex (as seen from downstream of the front looking towards upstream) are shock-free, whereas those which are concave (again as seen from downstream looking upstream) can produce shocks. The basis of this result can be obtained with reference to Figure 4 .

Firstly, we consider two initially straight sections of front joined at a convex corner (Figure $4(\mathrm{a})$ ) with included angle $\theta_{\text {incl }}$. We suppose this corresponds to time $t_{\text {corner }}$. We focus in on a small distance scale in the neighbourhood of the corner (so that $\Delta P^{\prime}$ is effectively constant). Likewise we focus in on a short time scale evolution following time $t_{\text {corner }}$, so again $s^{\prime}$ may be treated as constant. Any point on the front, whether on the straight sections or exactly at the corner, moves at the same constant speed $\Delta P^{\prime} / s^{\prime}$.

At some time $t^{\prime}$ very soon after $t_{\text {corner }}$ all points will have displaced by an amount $\left(\Delta P^{\prime} / s^{\prime}\right)\left(t^{\prime}-t_{\text {corner }}\right)$ : the straight sections remain straight whereas the corner (initially just a point) has now given rise to a circular arc of curvature radius $\left(\Delta P^{\prime} / s\right)\left(t^{\prime}-t_{\text {corner }}\right)$, and subtending an angle $\pi-\theta_{\text {incl }}$. Clearly at the point where the arc joins the straight sections of curve there is a very mild singularity: not a discontinuity in tangent vector, but instead a discontinuity in curvature. Such a discontinuity would not be permitted to persist in the 'parabolic' viscous froth model with any finite $\gamma^{\prime}$ (where diffusion of curvature is active) but is permitted to persist here with $\gamma^{\prime}=0$.

We now consider the evolution of a concave shape (Figure 4(b)). We take as before two straight sections of front inclined at an angle $\theta_{\text {incl }}$ to one another, and joined at some time $t_{\text {arc }}$ by a small arc of curvature radius $r_{\text {arc } 0}$ subtending $\pi-\theta_{\text {incl }}$. We suppose that 
$r_{\text {arc } 0}$ is exceedingly small (compared to the total depth across which the foam front can propagate), and so focus on a spatial region across which $\Delta P^{\prime}$ is effectively constant, and we also follow the evolution for a very short time such that $s^{\prime}$ is effectively constant. The arc continues to subtend an angle $\pi-\theta_{\text {incl }}$ but (as time $t^{\prime}$ increases) its radius $r_{\text {arc }}$ shrinks as $r_{a r c}=r_{\text {arc } 0}-\left(\Delta P^{\prime} / s^{\prime}\right)\left(t^{\prime}-t_{\text {arc }}\right)$. At time $t_{\text {arc }}+\left(s / \Delta P^{\prime}\right) r_{\text {arc } 0}$ the arc has shrunk completely away to a sharp corner, i.e. a singularity where the tangent to the front is discontinuous. This then begs the question of how the system evolves after the sharp corner is formed, an issue we will return to later.

For the present however we observe that the numerical simulations for pressure-driven growth of a foam front in a reservoir already performed by Shan \& Rossen (2004) and de Velde Harsenhorst et al. (2014) clearly indicate front shapes that are convex - so we expect that shocks would be avoided. As was noted above (with reference to Figure 4(a)) this does not rule out the possibility of some other type of singularity occurring (perhaps more mild than a shock) - and such singularities do indeed arise (see Section 4).

The fact the equations themselves admit potentially shock-like solutions (even though the solution of main interest here does not exhibit them) has some consequences for numerical schemes, and these are addressed later in Section 5. Before considering these issues however, we shall analyse the long-time asymptotic behaviour of the front shape.

\section{Asymptotic behaviour of front shape}

Numerical solution of the equation (2.10) for pressure-driven growth, whilst far more economical than solving the viscous froth model (3.3) with a small but finite $\gamma^{\prime}$, nevertheless presents challenges. The longer the time for which material is injected, the greater the total length of the foam front, corresponding to a large multitude of numerical grid points, and hence potentially expensive simulations, which may moreover confront numerical instability issues (de Velde Harsenhorst et al. 2014). Long-time behaviour of the solutions to equation (2.10) was a regime which Shan \& Rossen (2004) and de Velde Harsenhorst et al. (2014) left unexplored. It is desirable to find out if there is an asymptotic solution for the shape of the foam front without having to simulate numerically all the way out to long times. Particularly in view of the instabilities to which numerical simulations of this system are sensitive (de Velde Harsenhorst et al. 2014), finding some sort of analytic solution, even a long-time asymptotic one, is potentially extremely useful, both for benchmarking the numerics and for permitting a better understanding of the system.

The remainder of this section is laid out as follows. In Section 4.1, we derive the equations governing the long-time asymptotic behaviour of the front shape, and present the solution. The sections following that proceed to analyse this solution. Specifically Section 4.2 derives a result for the amount of area that remains unswept when the top of the foam front arrives at a production well: evaluating this unswept area is important in a petroleum engineering context. Sections 4.3 and 4.4 analyse the local shape of the front respectively near its bottom and top end. In particular, we show there can be a singularity (albeit a relatively mild one) at the top, where the curvature of the front diverges. This is found to have important implications for how closely-spaced material points on the front separate from one another over the course of time (Section 4.4.1), which impact in turn upon numerical schemes (Section 4.4.2).

\subsection{Long-time asymptotic solution}

At a large time $t^{\prime} \gg 1$, we know from equation (2.17) that the top of the foam front $\left(y^{\prime}=1\right.$ ) has displaced to $x^{\prime}=s^{\prime}=\sqrt{2 t^{\prime}}$ (the value $s_{0}^{\prime} \ll 1$ in equation (2.17) now being insignificant). As $y^{\prime}$ decreases, $x^{\prime}$ decreases also, but for almost all $y^{\prime}$ values (see 
note $(i)), \sqrt{2 t^{\prime}}-x^{\prime}$ will be much smaller than $\sqrt{2 t^{\prime}}$. The same rule applies for $s^{\prime}$ values for almost all $y^{\prime}$ values, $\sqrt{2 t^{\prime}}-s^{\prime}$ will be much smaller than $\sqrt{2 t^{\prime}}$. This is true despite the fact that $s^{\prime}$ does not just depend on the instantaneous $x^{\prime}$ and $y^{\prime}$ location of a material point, but in principle on the entire path history that the point has executed. The reason for the lack of sensitivity to path history is that when a point has displaced a long way downstream horizontally (order $\sqrt{2 t^{\prime}}$ with $t^{\prime} \gg 1$ ), any vertical displacement (up to order unity) makes only a second order contribution to the path length. In other words, the path length travelled is well approximated by the horizontal displacement alone, with any corrections from the vertical displacement being second order in the (small) ratio between vertical and horizontal displacements.

Based on the above discussion, our long-time asymptotic solution obeys

$$
\frac{\mathrm{d} \boldsymbol{x}^{\prime}}{\mathrm{d} t^{\prime}}=\frac{\Delta P^{\prime}}{\sqrt{2 t^{\prime}}} \boldsymbol{n}
$$

This is simpler than equation (2.10), since here the denominator $\sqrt{2 t^{\prime}}$ is spatially uniform, whereas in equation (2.10), the analogous denominator term $s^{\prime}$ depended on position as well as time. Expressing the above equation in Cartesian coordinates gives

$$
\begin{aligned}
& \frac{\mathrm{d} x^{\prime}}{\mathrm{d} t^{\prime}}=\frac{y^{\prime} \sin \alpha_{c}}{\sqrt{2 t^{\prime}}}, \\
& \frac{\mathrm{d} y^{\prime}}{\mathrm{d} t^{\prime}}=-\frac{y^{\prime} \cos \alpha_{c}}{\sqrt{2 t^{\prime}}} .
\end{aligned}
$$

We define a similarity variable of the form

$$
\xi=x^{\prime}-\sqrt{2 t^{\prime}}
$$

and look for a similarity solution with $y^{\prime}$ as a function of $\xi$ (or $\xi$ as a function of $y^{\prime}$ ).

At time $t^{\prime}+\mathrm{d} t^{\prime}$ the material point has displaced to

$$
\left(\xi+\mathrm{d} \xi, y^{\prime}+\mathrm{d} y^{\prime}\right)=\left(\xi+\frac{\left(y^{\prime} \sin \alpha_{c}-1\right)}{\sqrt{2 t^{\prime}}} \mathrm{d} t^{\prime}, y^{\prime}-\frac{y^{\prime} \cos \alpha_{c}}{\sqrt{2 t^{\prime}}} \mathrm{d} t^{\prime}\right) .
$$

The formula for the front is therefore

$$
\frac{\mathrm{d} y^{\prime}}{\mathrm{d} \xi}=\frac{y^{\prime} \cos \alpha_{c}}{1-y^{\prime} \sin \alpha_{c}} .
$$

Since

$$
\cos \alpha_{c}=\frac{1}{\sqrt{1+\left(\mathrm{d} y^{\prime} / \mathrm{d} \xi\right)^{2}}}, \quad \sin \alpha_{c}=\frac{\mathrm{d} y^{\prime} / \mathrm{d} \xi}{\sqrt{1+\left(\mathrm{d} y^{\prime} / \mathrm{d} \xi\right)^{2}}},
$$

it is possible to deduce

$$
\frac{\mathrm{d} y^{\prime}}{\mathrm{d} \xi}=\frac{y^{\prime}}{\sqrt{1-y^{\prime 2}}}
$$

The integral is

$$
-\xi=-\sqrt{1-y^{\prime 2}}+\log \frac{1}{y^{\prime}}+\log \left(1+\sqrt{1-y^{\prime 2}}\right)
$$

which has been written in this particular form to remind us that $\xi<0$ in the domain of interest. Via some difference of squares identities, this can also be written

$$
-\xi=-\sqrt{1-y^{\prime 2}}+\frac{1}{2} \log \left(\frac{1+\sqrt{1-y^{\prime 2}}}{1-\sqrt{1-y^{\prime 2}}}\right) .
$$


We have plotted the shape of this long-time asymptotic solution in Figure 5, and it certainly shows a convex shape. In Figure 5(a) we directly compare the asymptotic shape with finite time numerical results from de Velde Harsenhorst et al. (2014). Agreement is surprisingly good in the upper half of the solution domain, but in the lower half is imperfect, although that is unsurprising (remembering that the numerical results were obtained at a comparatively modest time $t^{\prime}=8$ only - see note $(j)$ ). Despite the imperfect agreement, the fit between the $t^{\prime}=8$ numerical solution and the long-time asymptotic result is far better than that between the numerics and an early-time solution given by de Velde Harsenhorst et al. (2014): this early-time solution only applies if points on the front have not moved any significant vertical distance during their entire evolution to date, a situation which is simply not the case (see note $(k)$ ) by time $t^{\prime}=8$. Agreement is far better by times $t^{\prime}=20$ and $t^{\prime}=30$ as Figure $5(\mathrm{~b})$ also shows.

Given the general agreement between long-time asymptotics and numerical results, in the subsections to follow, we analyse the formulae (4.9) and/or (4.10) in more detail.

\subsection{Asymptotic unswept area}

From the above solution, it is possible to demonstrate that

$$
\int_{0}^{1}-\xi \mathrm{d} y^{\prime}=\frac{\pi}{4} .
$$

This is an important result as it represents the 'unswept area' of the system. If foam is injected down to a certain fixed depth in an injection well, and subsequently flows and reaches a production well far downstream, then a well defined amount of area between the injection and production wells is yet to be reached by foam. The generalization from a 2-dimensional to an axisymmetric system is extremely straightforward (see note $(l)$ ).

The fact that $\int_{0}^{1}-\xi \mathrm{d} y^{\prime}$ remains finite even for arbitrarily large $t^{\prime}$ also corroborates an observation of de Velde Harsenhorst et al. (2014), namely that foam propagating simultaneously along and downwards into a reservoir helps to prevent gravity segregation i.e. override. Finite $\int_{0}^{1}-\xi \mathrm{d} y^{\prime}$ reflects the fact that (at almost all heights $y^{\prime}$, with the only exception being $y^{\prime} \ll 1$ ) the $\xi$ location of the foam front is at most an order unity amount behind the leading edge at the top boundary of the front (see note $(m)$ ), even though that leading edge has displaced through $\sqrt{2 t^{\prime}}$ with $t^{\prime} \gg 1$ here.

Sweep is predicted to be even better for real reservoirs, which are much less deep than a dimensionless depth of unity (recall from equation (2.8) how our depth scale has been defined). For instance, the example reservoir described by de Velde Harsenhorst et al. (2014), corresponds to $0.9865 \leqslant y^{\prime} \leqslant 1$. According to equation (4.13) the bottom of the front lags behind the top by only a distance $|\xi|=0.00148$, although the front itself has travelled by a much larger distance: up to $x^{\prime}=4$ by the time $t^{\prime}=8$ considered by de Velde Harsenhorst et al. (2014). All of these results indicating the high efficiency of reservoir sweep are immensely important from a petroleum engineering point of view.

\subsection{Shape near the bottom of the foam front}

For small $y^{\prime}$ (near the bottom of the foam front), we have $-\xi \sim-1+\log \left(2 / y^{\prime}\right)$, so that

$$
y^{\prime} \sim 2 \exp (-(1-\xi))
$$

As $\xi$ becomes large and negative (i.e. far behind the leading edge of the foam front), $y^{\prime}$ goes smoothly to zero at the bottom of the foam front, which is the expected behaviour. 


\subsection{Shape near the top of the foam front}

Near the top of the front, we find

$$
-\xi \approx \frac{2 \sqrt{2}}{3}\left(1-y^{\prime}\right)^{3 / 2}
$$

from which it follows for $y^{\prime} \sim 1$

$$
\frac{\mathrm{d} \xi}{\mathrm{d} y^{\prime}} \sim \sqrt{2\left(1-y^{\prime}\right)}
$$

whilst the curvature satisfies

$$
\kappa^{\prime} \equiv-\frac{\mathrm{d}^{2} \xi}{\mathrm{d} y^{\prime 2}} \sim \frac{1}{\sqrt{2\left(1-y^{\prime}\right)}}
$$

and so diverges.

Equations (4.13)-(4.14) have a number of important implications.

Firstly the tangent vector $\boldsymbol{t}$ changes direction very rapidly near the top boundary (see also Figure 5(c) which measures this direction change via the angle $\alpha$ ). If one were to represent this analytical solution for the front shape via a set of discrete points in a numerical simulation, it is necessary to have a very fine grid. On a coarse grid, the discrete points would appear to violate the top boundary condition $\alpha_{c}=\frac{\pi}{2}$ (even though the underlying analytical solution actually satisfies it). Evidence for this can be seen in the simulation data of Shan \& Rossen (2004) and de Velde Harsenhorst et al. (2014).

The second noteworthy point is that the curvature diverges (again see Figure 5(c)). This behaviour can occur for pressure-driven growth (equation (2.10)) but certainly not for a viscous froth model (equation (3.3)), because (for viscous froth) curvature always diffuses away from any such singularity. Indeed equation (4.9) is not a viable long-time asymptotic state for a viscous froth no matter how small the parameter $\gamma^{\prime}$. Divergent curvature at the top of the front coupled to any non-zero $\gamma^{\prime}$ would always cause the top of the front to recede, not to advance (for more details of this, refer to Appendix A).

The singularity represented by equations (4.13)-(4.15) is nonetheless milder than that at a sharp corner discussed previously (see e.g. Section 3.6 and Figure 4). Equation (4.14) turns the front tangent through a finite angle in a finite distance, whereas, at a sharp corner, the tangent would turn through a finite angle in an infinitesimal distance. One of the features of the convex corner illustrated in Figure 4(a) is that it causes material points which were initially 'coincident' to separate linearly in time. Another way of describing this is that a material point at a convex corner grows into an entire arc. It is interesting to investigate whether the singularity represented by equations (4.13)-(4.15) has similar properties governing the separation of material points: we explore this point below.

\subsubsection{Separation between material points}

Near $y^{\prime} \rightarrow 1$, equation (4.3) simplifies (using equations (4.7) and (4.14)) to

$$
\frac{\mathrm{d} y^{\prime}}{\mathrm{d} t^{\prime}} \sim-\sqrt{\frac{1-y^{\prime}}{t^{\prime}}} .
$$

This equation describes the motion of material points.

It is clear that a material point exactly on the top boundary $y^{\prime}=1$ at some arbitrary time $t_{\text {arb }}$ (assumed to satisfy $t_{\text {arb }} \gg 1$ ) can remain there indefinitely (which was in fact the rationale for choosing the boundary condition $\alpha_{c}=\frac{\pi}{2}$ there). Nevertheless a point which is instantaneously at some point $y_{\text {inst }}$ (which can be taken arbitrarily close to the 
top) at this same time $t_{\text {arb }}$ follows a trajectory

$$
y^{\prime} \sim 1-\left(\sqrt{t^{\prime}}-\sqrt{t_{\text {arb }}}+\sqrt{1-y_{\text {inst }}}\right)^{2} .
$$

If we define $T$ to be the time elapsed since $t_{a r b}$, i.e.

$$
T=t^{\prime}-t_{a r b},
$$

and Taylor expand equation (4.17) in $T$ assuming $\left(1-y_{\text {inst }}\right) \ll T^{2} / t_{\text {arb }} \ll 1$, we deduce

$$
y^{\prime} \sim 1-\frac{T^{2}}{4 t_{a r b}} .
$$

Equation (4.19) is only true for a limited domain of $T$ values, $T \ll \sqrt{t_{a r b}}$, since equation (4.17) itself (from which (4.19) has been derived) is only valid for $y^{\prime}$ close to 1 . Nevertheless it is clear that (as a result of the singularity) closely-spaced points are separating from one another, albeit quadratically in time (rather than linearly in time as would happen for an initial convex corner).

Although we must be cautious about extrapolating equation (4.19) outside its formal $1-y^{\prime} \ll 1$ domain of validity, it does suggest that material points injected from the top boundary onto the front at time $t_{a r b}$ (with $t_{a r b} \gg 1$ ) make the transition from top to bottom within a time $O\left(\sqrt{t_{\text {arb }}}\right)$.

This is also consistent with equation (4.3) which shows that away from the bottom boundary (where $y^{\prime} \ll 1$ ) and away from the top boundary (where $\cos \alpha_{c} \ll 1$ ), vertical motion proceeds at an $O\left(1 / \sqrt{t_{a r b}}\right)$ speed, and so points cover the unit distance from top to bottom in an $O\left(\sqrt{t_{a r b}}\right)$ time.

Thus at time $t_{a r b} \gg 1$, many of the points that we currently see on the foam front (with the exception of those in the domain $y^{\prime} \ll 1$ ), have been injected onto the front in the comparatively recent past, i.e. within the previous $O\left(\sqrt{t_{\text {arb }}}\right)$ time interval (see note $(n)$ ). Points injected any earlier than that, are already virtually at the bottom boundary.

The foregoing asymptotic analysis has important implications for a numerical scheme for solving the pressure-driven growth equations at finite time. Numerical grid points, even if placed arbitrarily close to the top boundary on an arbitrarily fine grid, migrate a finite distance away from the boundary with time: indeed de Velde Harsenhorst et al. (2014) noted this grid point migration in their study. Regridding is inevitable (see note $(o))$. Placement of new grid points is however non-trivial as we discuss below.

\subsubsection{Implications for new point placement in a numerical scheme}

Suppose that one knows a grid point $\left(x_{t o p}, y_{\text {top }}\right) \equiv\left(\sqrt{2 t^{\prime}}, 1\right)$ exactly on the top boundary, and (on a coarse mesh) has a grid point below it $\left(x_{\text {coarse }}, y_{\text {coarse }}\right)$, and wants to place a refined grid point at some position $\left(x_{\text {fine }}, y_{\text {fine }}\right)$, where the value of $y_{\text {fine }}$ is specified (see note $(p)$ ) between $y_{\text {coarse }}$ and $y_{\text {top }}$. Then to respect the local front shape near the top as defined by equation (4.13) we could expect to place $x_{\text {fine }}$ at

$$
x_{\text {fine }}=x_{\text {top }}-\left(x_{\text {top }}-x_{\text {coarse }}\right) \frac{\left(y_{\text {top }}-y_{\text {fine }}\right)^{3 / 2}}{\left(y_{\text {top }}-y_{\text {coarse }}\right)^{3 / 2}} .
$$

We also need to assign a path length $s_{\text {fine }}$ to the newly created point (given known path lengths $s_{t o p}$ and $s_{\text {coarse }}$ ) and consistently with equation (4.20) we can define

$$
s_{\text {fine }}=s_{\text {top }}-\left(s_{\text {top }}-s_{\text {coarse }}\right) \frac{\left(y_{\text {top }}-y_{\text {fine }}\right)^{3 / 2}}{\left(y_{\text {top }}-y_{\text {coarse }}\right)^{3 / 2}} .
$$


Point placement following equations (4.20)-(4.21) will ensure that plots of $y^{\prime}$ vs $x^{\prime}$ will still look as though they meet the top boundary of the system with the correct boundary condition, i.e. angle $\alpha_{c}=\frac{\pi}{2}$ (as defined by equation (2.16)).

Clearly developing numerical schemes to simulate pressure-driven growth is subtle, owing to the physical nature of the equations themselves, which can admit singularities in the solutions. More details about numerical schemes are discussed in the next section.

\section{Numerical analysis and results}

Robust numerical treatment of pressure-driven growth has two distinct requirements:

(a) Adequate representation of singularities when the governing equations indicate they are present, and

(b) Containment of a spuriously introduced singularity to prevent it spreading into regions of a solution domain from which singularities should be absent.

We have already discussed an example of requirement $(a)$ in the previous section. Here instead we consider requirement $(b)$.

We have solved equations (2.10)-(2.11) and (2.13) using Surface Evolver (and, for independent confirmation, separately in both $\mathrm{C}$ and Matlab) using a dimensionless time step $\delta t^{\prime}$ of $5 \times 10^{-5}$, an initial value $s_{0}^{\prime}$ of 0.01 , and a maximum segment length of 0.05 (with initial segment length being half the maximum). Various refinements were introduced to the scheme. We calculate the normal vectors at each grid point (where two adjacent segments meet) by first computing the normal on each segment, and then taking a weighted average, weighting toward the shorter segment. Time-stepping was done using Heun's method, and new grid points were introduced according to the method described by equations (4.20)-(4.21).

Whilst by and large the algorithm performed well (in line with the findings of Shan \& Rossen (2004) and de Velde Harsenhorst et al. (2014)) and numerics agreed favourably with the asymptotic solutions (see Figure 5), occasionally numerical errors (e.g. round-off and/or truncation errors) could cause a numerical point to fall behind its neighbours, thereby introducing a local concavity in an otherwise convex front shape. Over time, these local concavities could focus down into a kink.

Figure 6 shows a case where (similar to a methodology proposed by de Velde Harsenhorst et al. (2014)) we deliberately introduced (in this case at time $t^{\prime}=0.05$ ) such a local concavity, by moving a data point close to the top boundary (see also note $(q)$ ) to a position a horizontal distance roughly 0.001 to the right of its correct position (thereby introducing a very weak concavity as seen by the next point down). The subsequent evolution at times ranging up to $t^{\prime}=2.3$ does indeed show the development of a kink.

At time $t^{\prime}=1$ the concavity has still not developed into a visible kink: this seems to corroborate an observation by de Velde Harsenhorst et al. (2014) that a very low level of noise introduced into a front shape will require quite some time to develop into a kink (see note $(r)$ ). Nevertheless by time $t^{\prime}=2$ or $t^{\prime}=2.1$ a clear kink has formed, and has migrated a long way down from the top boundary. Between times $t^{\prime}=2.2$ and $t^{\prime}=2.3$ the numerics predict an infeasible and spurious topology for the front: material elements either side of the kink have crossed over one another to form a loop (the reasons that these spurious loops appear are discussed in Appendix B).

Ways to regularise the numerics, avoiding spurious behaviour, are considered below. Specifically in Section 5.1 we solve a viscous froth model with a non-zero but very weak surface tension term, and demonstrate that the results correspond to a regularised version of those for pressure-driven growth. It is shown that selection of an appropriate value of the surface tension parameter in the viscous froth model should be based on the small 
but finite thickness of the low mobility, wet foam zone at the foam front. In Section 5.2, we add a weak numerical diffusion term to the equations for pressure-driven growth. The resulting equations are shown to be analogous to those for the viscous froth. Such a result is unsurprising, given that the surface tension term within viscous froth is already known to be diffusive in nature (Satomi et al. 2013): whether the diffusion is physical in nature (Section 5.1) or numerical (Section 5.2) does not affect the end result. Section 5.3 meanwhile adopts an alternative approach: it does not introduce any diffusive term, so permits in principle singularities to appear in a pressure-driven foam front. However Section 5.3 incorporates a special rule for handling any singularities that might occur, which ensures that they propagate in a geometrically sensible fashion, consistent with the neighbouring pressure-driven growth.

\subsection{Regularising the system via a viscous froth model}

The discussion of Section 3 suggests that the system behaviour can be regularised by introducing a weak surface tension $\gamma^{\prime}$ via the viscous froth model. The question then arises of what should be an appropriate choice for the parameter $\gamma^{\prime}$.

We answer this question by introducing the concept of a 'maximum curvature' for the front. Treating the low mobility wet foam zone near a foam front as being infinitesimally thin is an idealisation. In the work of Shan \& Rossen (2004) and de Velde Harsenhorst et al. (2014) this zone neighbouring the front is not supposed to be infinitesimally thin but rather has a finite thickness $\tau_{\text {thick }}^{\prime}$ given by equation (2.12). It is not possible to bend an object of thickness $\tau_{\text {thick }}^{\prime}$ into a curvature radius of less than $\tau_{\text {thick }}^{\prime}$ (see e.g. Figure 7 and see also Kern \& Weaire (2003)): maximum curvature $\kappa_{\max }$ is therefore $\tau_{\text {thick }}^{\prime-1}$.

Now we expect that a suitable choice for $\gamma^{\prime}$ would be up to the order of $\kappa_{\text {max }}^{-1} \equiv \tau_{\text {thick }}^{\prime}$, since at that level (even for a curvature $\kappa_{\max }$ ) the surface tension-curvature term in equation (3.3) should still manage to be dominated by driving pressure, which is the essence of the pressure-driven growth model.

Assuming we choose

equation (3.3) becomes

$$
\gamma^{\prime}=\tau_{\text {thick }}^{\prime}
$$

$$
\frac{\mathrm{d} \boldsymbol{x}^{\prime}}{\mathrm{d} t^{\prime}}=\left(\frac{\Delta P^{\prime}}{s^{\prime}}-\tau \kappa^{\prime}\right) \boldsymbol{n}
$$

where $\tau$ is the parameter given in equation (2.5) and/or (2.12). We assume here that $\tau$ is a small parameter (which corresponds, as mentioned previously, to a foam that collapses readily as it dries out).

Numerical solution of equation (5.2) involves some additional considerations over and above those for pressure-driven growth. Primarily these concern the methods in which boundary conditions are implemented, as we now explain.

At the top boundary, one could set $\alpha=0$ or equivalently $\alpha_{c}=\frac{\pi}{2}$ (where $\alpha$ and $\alpha_{c}$ are angles defined in Figure $2(\mathrm{~b})$ ). At each time step this would require updating the positions on all 'internal' front points, i.e. those away from the top boundary, and then finding the top point itself, by fitting a smooth curve through the nearest and next nearest data points adjacent to the top, whilst constraining the value of $\alpha$ at the point where this smooth curve intersects the top boundary (see note $(s)$ ). This would determine a curvature at the top boundary, leading to slightly slower motion of points on the top boundary for a viscous froth model than for pressure-driven growth. A simpler alternative for a viscous froth simulation is to assume points on the boundary migrate according to equation (2.17), the same motion as applies for pressure-driven growth.

Similar considerations apply at the bottom boundary. One could assume that the front 
spreads out symmetrically on both left and right, and thereby impose a constraint that $\mathrm{d} y^{\prime} / \mathrm{d} x^{\prime}=0$ at $x^{\prime}=0$. In this case the front at $x^{\prime}=0$ will need to find its own $y^{\prime}$ level (e.g. by fitting a smooth curve through nearest neighbour and next nearest data points, constraining the curve so that $\left.\mathrm{d} y^{\prime} /\left.\mathrm{d} x^{\prime}\right|_{x^{\prime}=0}=0\right)$, the level attained being hopefully close to $y^{\prime}=0$ if the parameter $\tau$ is small. The problem however becomes the choice of initial condition: at present we assume the front is initially vertical (between $\left(x^{\prime}, y^{\prime}\right)=\left(s_{0}^{\prime}, 1\right)$ and $\left(x^{\prime}, y^{\prime}\right)=\left(s_{0}^{\prime}, 0\right)$ for some $\left.s_{0}^{\prime} \ll 1\right)$, but in order to satisfy $\mathrm{d} y^{\prime} / \mathrm{d} x^{\prime}=0$ at $x^{\prime}=0$, the vertical initial state must be made to turn sharply near the bottom, specifically in the domain $0 \leqslant x^{\prime} \leqslant s_{0}^{\prime}$, so as to become horizontal at $x^{\prime}=0$. Depending on how sharply the front is made to turn, curvature could drag nearby points upwards at different rates. As the front evolves over time however, curvature should diminish again, so the points in the neighbourhood of $x^{\prime}=0$ should also settle back towards $y^{\prime}=0$ at long times. An alternative that avoids these complications is to start with a vertical front as before, and simply to pin the point at the bottom of the front at e.g. $\left(x^{\prime}, y^{\prime}\right)=\left(s_{0}^{\prime}, 0\right)$ for $s_{0}^{\prime} \ll 1$ (which is what effectively occurs also in the case of pressure-driven growth).

Our principal aim here is to use equation (5.2) to examine the effect of deliberately introducing a local concave defect in an otherwise convex solution. To achieve this aim, it is sufficient to choose the 'simpler' boundary condition at either end, i.e. equation (2.17) at the top, and a pinning condition at the bottom. Subject to those boundary conditions, we solved equation (5.2) with a $\tau$ value, $\tau=0.01$. A concave defect of size 0.001 was introduced just below the top boundary at a time $t^{\prime}$ of 0.0352 . The range of permitted segment lengths utilised in the simulation was similar to before (i.e. up to 0.05 maximum, with initial segment length 0.03125$)$ and the time step was also similar $\left(2 \times 10^{-5}\right)$. We also checked for convergence by reducing segment length tenfold (maximum segment lengths now 0.005 ) and reducing time step by a hundredfold (now $2 \times 10^{-7}$ ): if data for front shapes at selected times up to $t^{\prime}=3.0$ were plotted graphically, the graphs were indistinguishable regardless of whether we used the smaller segment lengths and small time steps or their larger counterparts. The small segment length and small time step calculations were expensive however (9 hours to run on an i3 CPU to reach $t^{\prime}=1.0$ ) so we reverted back to the larger segment lengths and larger time steps.

Results are shown in Figure 8 for the case of permitted segment lengths up to 0.05 and time step $10^{-5}$. The curvature of the concave region introduced at time 0.0352 helped to increase its speed relative to its neighbours. It was thus (by the times shown on Figure 8) able to catch up with its neighbours and heal the defect.

The good numerical behaviour of the system, even with comparatively large spatial intervals and/or time steps, is remarkable, given we know that the analogous pressuredriven growth system admits a singularity at the top boundary at long times (see Section 4), and this singularity must be relieved in a small neighbourhood of the top boundary (see also Appendix A). Apparently the fine details of how the solution matches onto the top boundary (which would only be captured accurately with a sufficiently small numerical spatial step) have limited influence upon the rest of the solution domain.

If however we were to reduce the parameter $\tau$ still further, keeping the same range of spatial intervals and the same time step, numerical problems do arise, albeit not at the top boundary. We considered values for $\tau$ of $10^{-3}, 10^{-5}, 10^{-7}$ and $10^{-10}$. Detailed data are not given here, but for values of $10^{-5}$ and below, spurious topologically infeasible loops (like those in Figure 6) begin to appear (see also Appendix B). The reason for this is clear. For a given minimum size spatial interval, the maximum curvature that any numerical simulation can access scales proportionally to the reciprocal of the interval size. If this given maximum accessible curvature is multiplied by smaller and smaller $\tau$ values, we eventually return to a situation where the dynamics of the system are completely 
dominated by pressure-driven growth. Only by permitting yet smaller spatial intervals can we increase the maximum curvature that a numerical simulation accesses, and allow curvature-driven dynamics on a concavity to overcome any sharpening tendency produced by pressure-driven growth. However for a value of $\tau=10^{-5}$ (or below) where very tiny spatial intervals would be required, we have an exceedingly stiff numerical system.

\subsection{Regularising the system via numerical diffusion}

Yet another possible way to regularise the system behaviour is not to introduce a physical diffusion (via surface tension) but rather to introduce a numerical diffusion, by replacing the nominal velocity of a point on the front by a weighted sum of its own velocity and that of its neighbours, and then projecting this weighted sum onto the correct propagation direction. Schemes like this have been used by de Velde Harsenhorst et al. (2014).

Assuming a point at arc length location $S^{\prime}$ measured along the front, and a grid spacing $\delta S^{\prime}$ (small enough that $\Delta P^{\prime}$ and $s^{\prime}$ are nearly constant)

$$
\begin{aligned}
\frac{\mathrm{d} \boldsymbol{x}^{\prime}}{\mathrm{d} t^{\prime}}=\frac{1}{s^{\prime}}\left(\frac{\Gamma^{\prime}}{\delta S^{\prime 2} \kappa^{\prime}} \boldsymbol{n}\left(S^{\prime}-\delta S^{\prime}\right)+\left(\Delta P^{\prime}-2 \frac{\Gamma^{\prime}}{\delta S^{\prime 2} \kappa^{\prime}}\right) \boldsymbol{n}\right. \\
\left.\quad+\frac{\Gamma^{\prime}}{\delta S^{\prime 2} \kappa^{\prime}} \boldsymbol{n}\left(S^{\prime}+\delta S^{\prime}\right)\right) \cdot \boldsymbol{n} \boldsymbol{n} \\
=\frac{\Delta P^{\prime}}{s^{\prime}}\left(\frac{\Gamma^{\prime}}{\delta S^{\prime 2} \Delta P^{\prime} \kappa^{\prime}} \boldsymbol{n}\left(S^{\prime}-\delta S^{\prime}\right)+\left(1-2 \frac{\Gamma^{\prime}}{\delta S^{\prime 2} \Delta P^{\prime} \kappa^{\prime}}\right) \boldsymbol{n}\right. \\
\left.\quad+\frac{\Gamma^{\prime}}{\delta S^{\prime 2} \Delta P^{\prime} \kappa^{\prime}} \boldsymbol{n}\left(S^{\prime}+\delta S^{\prime}\right)\right) \cdot \boldsymbol{n} \boldsymbol{n}
\end{aligned}
$$

where $\Gamma^{\prime}$ is a weighting factor that we must specify and where $s^{\prime}$ and $\kappa^{\prime}$ are computed at point $S^{\prime}$, as is $\boldsymbol{n}$ (unless otherwise specified via giving $\boldsymbol{n}$ an argument $S^{\prime} \pm \delta S^{\prime}$ ). Note that equation (5.3) appears to be singular in the initial state which has $\kappa^{\prime}=0$, but in that state also $\boldsymbol{n}\left(S^{\prime} \pm \delta S^{\prime}\right)=\boldsymbol{n}$, so equation (2.10) is regained. Provided $\Gamma^{\prime}$ is not too large (to admit the use of a Taylor expansion), equation (5.3) approximates to

$$
\frac{\mathrm{d} \boldsymbol{x}^{\prime}}{\mathrm{d} t^{\prime}} \approx \frac{\Delta P^{\prime}}{s^{\prime}}\left(\boldsymbol{n}\left(S^{\prime}\right)+\frac{\Gamma^{\prime}}{\Delta P^{\prime} \kappa^{\prime}} \frac{\mathrm{d}^{2} \boldsymbol{n}}{\mathrm{d} S^{\prime 2}}\right) \cdot \boldsymbol{n} \boldsymbol{n}=\frac{\left(\Delta P^{\prime}-\Gamma^{\prime} \kappa^{\prime}\right)}{s^{\prime}} \boldsymbol{n}
$$

where we have used the identity that $\boldsymbol{n} \cdot \mathrm{d}^{2} \boldsymbol{n} / \mathrm{d} S^{\prime 2}=-\kappa^{\prime 2}$. This then is identical to the viscous froth model (and hence to the data in Figure 8) provided we make the association

$$
\gamma^{\prime} \longleftrightarrow \Gamma^{\prime}
$$

For this numerical diffusion scheme, accurately resolving front shapes in any regions of 'maximal curvature' $\kappa_{\max }=1 / \tau_{\text {thick }}^{\prime}$ requires a spatial step

$$
\delta S^{\prime} \sim \kappa_{\max }^{-1}=O(\tau)
$$

where $\tau$ is the small parameter identified in equation (2.12), and path lengths $s^{\prime}$ are assumed order unity here.

Leaving such high curvature regions aside however, and focussing instead on regions where $\kappa^{\prime}$ is order unity, equation (5.3) is close to the original pressure-driven growth equation (2.10) provided $\delta S^{\prime} \geqslant O\left(\Gamma^{\prime 1 / 2}\right)$. If we choose $\Gamma^{\prime}=O(\tau)$ as equations $(2.12)$, (5.1) and (5.5) tend to suggest (assuming path lengths $s^{\prime}$ of order unity, and still with $\tau$ being a small parameter), then we could easily have much larger spatial steps,

$$
\delta S^{\prime} \sim O\left(\tau^{1 / 2}\right)
$$

although regions of maximal curvature would then remain improperly unresolved. 
The techniques described above avoid spurious development of kinks from local concavities in front shapes by introducing some kind of diffusion (either 'physical diffusion' or 'numerical diffusion'). This begs the question of whether there might be some other 'non-diffusive' modification to the pressure-driven growth system which still manages to avoid focussing any concavities down to a kink: these are considered in what follows.

\section{3. 'Non-diffusive' modifications to pressure-driven growth}

Figure 9 shows a (discretised version of) a concave segment of front with discrete points $\boldsymbol{x}_{n-1}^{\prime}, \boldsymbol{x}_{n}^{\prime}$ and $\boldsymbol{x}_{n+1}^{\prime}$. Each discretised segment has length $\delta S^{\prime}$ and the front turns through an angle $\delta \theta$ over this same distance $\delta S^{\prime}$ (measured from the midpoint of one discrete segment to the midpoint of the next).

As the structure advances at speed $u^{\prime} \equiv \Delta P^{\prime} / s^{\prime}$ over a time step $\delta t^{\prime}$, it is clear that a length of front $u^{\prime} \delta t^{\prime} \tan \frac{\delta \theta}{2}$ should be consumed from each segment - and the point $\boldsymbol{x}_{n}^{\prime}$ is in fact amongst the material thus consumed.

If however we propagate this point, not with its true speed $u^{\prime}$, but rather with an apparent speed $u_{a p p}^{\prime}=u^{\prime} / \cos \frac{\delta \theta}{2}$, then it is clear that the turning angle $\delta \theta$ between the two segments is conserved, rather than focussing down into sharper and sharper turns.

A new rule for pressure-driven growth rule then results

$$
\frac{\mathrm{d} \boldsymbol{x}^{\prime}}{\mathrm{d} t^{\prime}}=\left\{\begin{array}{cc}
\frac{\Delta P^{\prime}}{s^{\prime}} \boldsymbol{n}, & \kappa^{\prime}>0 \text { (convex) } \\
\frac{\Delta P^{\prime}}{s^{\prime} \cos \frac{\delta \theta}{2}} \boldsymbol{n}, & \kappa^{\prime}<0 \text { (concave) }
\end{array}\right.
$$

where the sign of $\kappa^{\prime}$ is used to distinguish convex regions from concave ones.

We have implemented equation (5.8) starting from the configuration containing a small concavity that is shown in Figure 6(a), and found that, simulating out to time $t^{\prime}=4.0$ at least, the system behaves well. This is a contrast from the case of equation (2.10) which in Figure 6(b), already produced spurious results around time 2.2 or 2.3. We do not plot the solutions of equation (5.8) here, since visually they look very similar to the viscous froth predictions in Figure 8. Our numerical simulations of either equation (2.10) or equation (5.8) track the time evolution of the curvature $\kappa^{\prime}$ of the concavity and also the jump in angle $\delta \theta$ between adjacent elements there. In both cases we introduce the concavity at time 0.05 with $\kappa^{\prime}=-0.97$ and $\delta \theta=0.024$. By time $t^{\prime}=1.0$ there is still only a modest difference between the predictions (curvature $\kappa^{\prime}=-5.0$ and angle $\delta \theta=0.106$ in the case of equation (2.10) and $\kappa^{\prime}=-3.9$ and $\delta \theta=0.086$ for equation (5.8)). Modest differences in predictions are expected as long as $\delta \theta$ is small, because the $\cos \frac{\delta \theta}{2}$ factor in equation (5.8) then exhibits only second order variation in $\delta \theta$. As equation (2.10) sharpens the concavity over time however, and hence $\delta \theta$ grows, differences between the two sets of predictions grow. Indeed for time $t^{\prime}=2.0$ we predict $\kappa^{\prime}=-34.0$ and $\delta \theta=0.71$ using equation (2.10) compared with $\kappa^{\prime}=-4.6$ and $\delta \theta=0.13$ from equation (5.8). Shortly after $t^{\prime}=2.0$ we only track curvatures for equation (5.8) (as we already know that spurious behaviour onsets in the case of equation (2.10)). We find $\kappa^{\prime}=-17.7$ and $\delta \theta=0.23$ for $t^{\prime}=3.0$, and $\kappa^{\prime}=-141$ and $\delta \theta=0.26$ when $t^{\prime}=4.0$. Unlike in the case of equation (2.10), these surprisingly large curvatures do not seem to trigger the onset of any numerical misbehaviour. Instead they merely seem to reflect segments turning through a finite angle with exceedingly short edge lengths (see note $(t)$ ). Were we then to implement a rule that edges below a critical size were removed from the simulation and merged with their neighbours, we could in effect remove the concavity altogether.

Equation (5.8) applies to a discretised representation of a foam front, and is potentially weakly sensitive to discretisation through the $\cos \frac{\delta \theta}{2}$ term. 
There may be situations more general than those we have considered to date (see Section 6 later) in which, as part of the physics, we actually want concavities to appear and to sharpen down to a corner, and then to propagate those corners along with the rest of the front. In this case we could propose

$$
\frac{\mathrm{d} \boldsymbol{x}^{\prime}}{\mathrm{d} t^{\prime}}=\left\{\begin{array}{cl}
\frac{\Delta P^{\prime}}{s^{\prime}} \boldsymbol{n}, & \text { either } \kappa^{\prime}>0 \text { or }\left(\kappa^{\prime}<0 \text { with } \delta \theta<\delta \theta_{\text {sharp }}\right) \\
\frac{\Delta P^{\prime}}{s^{\prime} \cos \frac{\delta \theta}{2}} \boldsymbol{n}, & \kappa^{\prime}<0 \text { with } \delta \theta \geqslant \delta \theta_{\text {sharp }}
\end{array}\right.
$$

where $\delta \theta_{\text {sharp }}$ is a model parameter that we set (the sharpest permitted turning angle, beyond which material is treated as being focussed down into a corner).

Equation (5.9) (which is only a very minor change compared to equation (5.8)) represents a convenient way to describe possible sharp corners appearing during pressuredriven growth (see e.g. Figure 4(b) and Figure 9) without dramatically changing the physics underlying equation (2.10).

Front material points still invariably propagate with speed $\Delta P^{\prime} / s$. The fact that sharp corners propagate with a different (apparent) velocity merely signifies that they are not actually material points: indeed the material points in the neighbourhood of a corner are continually being consumed. Mathematically such behaviour near a corner can be considered as being equivalent to a wave optics problem (such as is often considered in studies of interference and/or diffraction (Knight 2008)), where two wavefronts propagating at the same speed but in slightly different directions are permitted to interact. An analogy with optics is not surprising given that we have already noted (see Section 3.5) the link between pressure-driven growth and the eikonal equation, which is very often studied in the context of optics (Arnold 2004).

Results from equation (5.9) are of course sensitive to the value of $\delta \theta_{\text {sharp }}$ that is chosen. Although it is mathematically feasible to have $\delta \theta_{\text {sharp }}$ values as large as $\pi$, in practice any value of $\delta \theta_{\text {sharp }}$ many times larger than $\delta S^{\prime}$ could be chosen as the signature of a sharp corner. Bearing in mind that there is a maximum possible curvature $\kappa_{\max }$ scaling as the reciprocal of a thickness $\tau_{\text {thick }}^{\prime}$, itself given by equation (2.12), one possible choice for $\delta \theta_{\text {sharp }}$ is $\delta \theta_{\text {sharp }} \sim \kappa_{\max } \delta S^{\prime} \equiv \delta S^{\prime} /\left(s^{\prime} \tau\right)$. That would however make a parameter in equation (5.9) sensitive to the choice of typical element length $\delta S^{\prime}$ and may also require prohibitively small element sizes $\delta S^{\prime}$ (significantly smaller than the already small parameter $\tau$ ) to maintain the constraint $\delta \theta_{\text {sharp }} \leqslant \pi$ : this is not so efficient numerically. A better option is to select a $\delta \theta_{\text {sharp }}$ value as a model parameter independently of the dis-

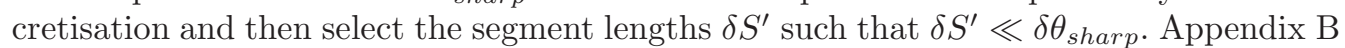
suggests that there are advantages in choosing $\delta S^{\prime} \ll \delta \theta_{\text {sharp }} \ll 1$.

\section{Discussion and outlook}

The formula given above, equation (5.9), provides a rule for propagating a foam front according to pressure-driven growth, permitting sharp corners, at least for an idealised situation where the front is treated as infinitesimally thin (see note $(u)$ ). Sharp corners only however arise in the case where the foam front exhibits concavities: they should not appear in the original case considered by Shan \& Rossen (2004) and de Velde Harsenhorst et al. (2014) where the front remains convex. Nevertheless there are three related problems (of relevance to foam IOR) where concavities (and hence sharp corners) could occur. Our purpose here is to describe these three problems, although we leave their detailed analysis for future work. The three problems in question are: 
(a) Liquid and/or surfactant slumping (Section 6.1),

(b) Increase in driving pressure (Section 6.2),

(c) A heterogeneous (stratified) reservoir (Section 6.3).

A summary is then given in Section 6.4.

\subsection{Liquid and surfactant slumping}

So called slumping occurs because liquid is heavier than gas - hence migrates downward in the reservoir. Also, although the density difference is much less, surfactant solution injected before gas may slump relative to water initially present. Over time, with liquid slumping, foam at the top of the reservoir becomes drier; with surfactant slumping, the foam at the top is less stable. Either process promotes bursting of foam films near the top. Realistically, there can be gas in the reservoir before injection of the surfactant slug, a complication not considered by Shan \& Rossen (2004) or de Velde Harsenhorst et al. (2014). In that case slumping of both liquid (i.e. water) and surfactant could be severe.

Since the mobility of the foam increases as the bubble size increases, we could model slumping (in an approximate fashion) by solving equation (2.10) up to a certain time $t_{\text {slump }}$ and thereafter solving

$$
\frac{\mathrm{d} \boldsymbol{x}^{\prime}}{\mathrm{d} t^{\prime}}=\left\{\begin{array}{cl}
\frac{\Delta P^{\prime}}{s^{\prime}}, & y^{\prime}<y_{\text {slump }} \\
\frac{M_{\text {slump }} \Delta P^{\prime}}{s^{\prime}}, & y_{\text {slump }}<y^{\prime} \leqslant 1
\end{array}\right.
$$

where $y_{\text {slump }}$ is the height to which the wetter part of the system slumps, and $M_{\text {slump }}>1$ is a factor representing the increased mobility of the drier region above (see Figure 10).

For $t^{\prime}>t_{\text {slump }}$, the Cartesian coordinate of the point at the top of the front $x^{\prime}$ and the corresponding path length travelled $s^{\prime}$ are now

$$
x^{\prime}=s^{\prime}=\sqrt{2 M_{\text {slump }}\left(t^{\prime}-t_{\text {slump }}\right)+2 t_{\text {slump }}+s_{0}^{\prime 2}} .
$$

It is clear that the more mobile foam front in the upper region $y_{\text {slump }}<y^{\prime}<1$ will now run ahead of the less mobile foam front lower down $y^{\prime}<y_{\text {slump }}$, which could introduce local concavities although front material points instantaneously in the neighbourhood of $y_{\text {slump }}$ will continually migrate from the upper to the lower region.

This certainly introduces difficulties into the solution of the present model, although it is actually far from clear that the model posed here properly captures all the possible detrimental effects of slumping. The most significant slumping is expected after liquid comes into contact with gas, whereas the present pressure-driven growth model focuses all the dynamics at that point of contact. It may then be important to consider slumping occurring well behind the advancing foam front. Insufficient surfactant solution high up in the reservoir well behind the foam front would be catastrophic for foam stability, enabling highly mobile gas to override a comparatively less mobile foam below. In order to model this however, we should be considering how the mobility evolves over the entire gas and foam bank, not focussing the dynamics merely on the front itself.

In summary, a pressure-driven growth model which assumes finite mobilities only at the foam front could potentially predict sharp-cornered foam front shapes in the presence of slumping, but ideally one would prefer to abandon the pressure-driven growth picture, and consider instead how slumping affects the variation of mobilities over the entire reservoir. There are however other situations (not involving slumping) where sharp-cornered foam front shapes are predicted, with pressure-driven growth still being expected to be a reasonable representation of the physics: these are discussed in Sections 6.2-6.3. 


\subsection{Increase in driving pressure}

The case of an increase in driving pressure supposes that at some time $t_{\text {press }}$ the pressure driving the foam is suddenly increased by a factor $1+p_{f a c}$. Equation $(2.11)$ is replaced after time $t_{\text {press }}$ by

$$
\Delta P^{\prime}=y^{\prime}+p_{f a c} .
$$

The top of the foam front at $y^{\prime}=1$ now satisfies for $t^{\prime}>t_{\text {press }}$

$$
x^{\prime}=s^{\prime}=\sqrt{2\left(1+p_{f a c}\right)\left(t^{\prime}-t_{\text {press }}\right)+2 t_{\text {press }}+s_{0}^{\prime 2}} .
$$

In addition to the equation (6.4), which applies at the top of the foam front, material deeper down in the system can now be displaced, so the domain of solution is now extended from $0 \leqslant y^{\prime} \leqslant 1$ to $-p_{f a c} \leqslant y^{\prime} \leqslant 1$. Newly created points in the domain $-p_{\text {fac }} \leqslant y^{\prime} \leqslant 0$ satisfy $x^{\prime}=s^{\prime}=s_{0}^{\prime}$ (at time $t^{\prime}=t_{\text {press }}$ ). It is clear (see Figure 11) that the front turns through a significant angle where the newly created domain intersects the original domain. Indeed the front could turn through close to a right angle if $t_{\text {press }}$ is sufficiently large - since then the front (immediately prior to time $t_{\text {press }}$ ) would be near horizontal close to the bottom of the original domain.

Given that a sharp corner is now an inherent part of the foam front, an evolution equation such as (5.9) will be required. It is even conceivable (albeit not certain) that the near horizontal section of front inherited from the original domain could develop additional concavities which subsequently focus into yet more corners. This could come about, because points on the near horizontal section which are further to the left (on Figure 11) have much smaller $s^{\prime}$ values than points further to the right: hence the points on the left migrate downwards at higher speeds than points on the right. This should produce concavities, but these may not necessarily have time to focus into yet more sharp corners, since rightward moving sections of front in the newly accessed part of the domain $-p_{f a c}<y^{\prime}<0$ potentially consume any concave regions that might start to form.

Regardless of whether just a single sharp corner is present, or whether that sharp corner is accompanied by additional concavities, it is clear that a step increase in pressure leads to mathematical complications in solving the pressure-driven growth equations compared to constant pressure operation. These complications can however be avoided by operating from the outset at maximum available pressure. There is however still a case where mathematical complications are inherent, and cannot be avoided merely by changing the mode of operation: this is addressed in Section 6.3.

\subsection{A heterogeneous (stratified) reservoir}

Yet another case of interest is a heterogeneous system (see note $(v)$ ) stratified into layers, where the permeability is modulated according to the depth. This could be described by

$$
\frac{\mathrm{d} \boldsymbol{x}^{\prime}}{\mathrm{d} t^{\prime}}=\frac{\left(1-k_{\text {strat }} \sin \left(2 \pi n_{\text {strat }}\left(1-y^{\prime}\right)\right)\right) \Delta P^{\prime}}{s^{\prime}} \boldsymbol{n}
$$

where $k_{\text {strat }}$ is a factor less than unity and $n_{\text {strat }}$ (which for convenience we think of as being an integer) represents the number of alternating low/high permeability layers.

At those locations where permeability is lower, the foam front could tend to lag behind places where permeability is higher (although see Appendix $\mathrm{C}$ for a more detailed discussion of which regions lag behind others). The foam front may then develop concavities which subsequently focus into sharp corners (see Figure 12). If this does occur, we would anticipate the front developing multiple sharp corners (one per low permeability layer).

In summary, even though the original pressure-driven growth problem considered by Shan \& Rossen (2004) and de Velde Harsenhorst et al. (2014) exhibited neither concavities 
nor sharp corners, there are some very natural extensions of that system, which are moreover of potential engineering relevance, where these features can arise. It is therefore very important that numerical schemes be developed to address them.

\subsection{Summary}

Section 5 considered a case where a spurious kink was introduced into a foam front through an erroneous perturbation superposed on the original front shape. Sections 6.16.3 on the other hand introduce cases where sharp corners appear in the front shape as an intrinsic part of the physics of the problem. Whilst these situations are distinct, the mechanism for dealing with them is essentially the same, i.e. the singular kink or corner is moved at a specially chosen velocity that keeps the singularity contained at a particular localized part of the front, with the chosen velocity of the singularity moreover being compatible with the motion of the surrounding parts of the front.

The important point here is not the singularity per se, but rather the fact that it is handled in a way that does not adversely affect the evolution of the rest of the front. In other words, if we were to relax from a singularity to a localised region of very large but finite curvature, the evolution of the overwhelming majority of locations on the front (i.e. away from the neighbourhood of a singularity and/or high curvature zone) remains unchanged. To this extent the front shapes we compute via pressure-driven growth are meaningful regardless of how a singularity might have originated, any singularity then just being an idealised representation of a sharply curved region that develops on the foam front.

\section{Conclusions}

We have used the pressure-driven growth model developed by Shan \& Rossen (2004) and de Velde Harsenhorst et al. (2014) to study propagation of a foam front during the process of foam IOR. We showed that the pressure-driven growth model (which balances driving pressure with viscous drag), is a special case of the so called viscous froth model (Glazier \& Weaire 1992; Weaire \& McMurry 1996) (which balances driving pressure, viscous drag and surface tension-curvature).

With our approach we derived asymptotic solutions for long times that are free of numerical instabilities; this asymptotic form fits the numerical results of de Velde Harsenhorst et al. (2014) for relatively long times well and shows that this front propagates indefinitely with no increase in gravity override. Thus this work both confirms and extends the partial results of de Velde Harsenhorst et al. (2014). We identified and explored the causes of the numerical instabilities seen by de Velde Harsenhorst et al. (2014), discussed how these instabilities arise and how to mitigate them, and found that they are inherent if the model is extended to heterogeneous reservoirs, changing injection pressure, and slumping of the surfactant solution. In cases where the instabilities are a natural result of the problem description, we obtained an evolution rule for modelling their propagation without exacerbating the instability. We presented better ways to interpolate points in the numerical solution for the front, especially near the top of the front, and to control numerical instabilities, than those proposed by de Velde Harsenhorst et al. (2014).

Specifically, our mathematical analysis of the foam IOR model in the context of the viscous froth model leads to the following results. The pressure-driven growth model considered here is a singular limit of the viscous froth model. Much of our analysis has concerned the origin of instabilities in the pressure-driven growth solution, which arise from concavities in the front. From a mathematical viewpoint, surface tension-curvature (within viscous froth) represents a diffusive term in the governing equation for foam 
front motion, which leads to curvature diffusing along foam fronts smoothing off any sharp corners. The pressure-driven growth model (from which diffusion is absent) can however admit solutions that contain sharp corners. Indeed pressure-driven growth is formally equivalent to the eikonal equation (Arnold 2004) which is known to permit such sharp-corner solutions. Equivalently from a physical viewpoint, surface tension represents an energy cost associated with the length of a foam film (or a foam front). The length of the film (or front) and hence the energy cost is reduced by rounding off corners. If however that energy cost is removed altogether (as in the IOR model of Shan \& Rossen (2004) or for a foam with identically zero surface tension), then no incentive remains to reduce length, and in principle sharp corners can then form and persist. A viscous froth model with a slight surface tension eliminates the instability problem; the concern is whether (and if so, to what extent) the surface tension affects the solution as a whole.

We have demonstrated that corners develop through focussing of concave sections of foam front. However the system specifically considered by Shan \& Rossen (2004) and de Velde Harsenhorst et al. (2014) actually has a front of convex shape.

This convex front shape is amenable to a long-time asymptotic analytical solution. This asymptotic solution matches the numerical solutions of de Velde Harsenhorst et al. (2014) well, in cases where their solutions are not distorted by obvious instability.

The existence of this long-time asymptotic solution indicates that in principle there is no limit to the distance over which a SAG process could prevent gravity segregation. (Segregation would require the leading edge of the front to run arbitrarily far ahead of points lower down, but the asymptotic solution indicates the leading edge remaining just a fixed distance ahead of points at a given depth lower down). Moreover the long-time solution predicts the area beneath the foam front that remains unswept as the top of the front reaches a production well. It is simply $\frac{\pi}{4}$ multiplied by the square of the maximum depth to which foam penetrates. This is of course a 2-dimensional result. In the case of axisymmetric flow about an injection well, the unswept volume is the product of the above mentioned unswept area, and the perimeter of a circle centred at the injection well and passing through the production well or wells..

While it does not contain any sharp corners per se, the long-time asymptotic solution exhibits a mild square root singularity at the top boundary of the reservoir. The asymptotic analytical solution reveals that the curvature of the front diverges at the singularity, which means that the front turns through a surprisingly large angle over a very short distance. A consequence of this is that material points on the front which start off arbitrarily close to the top boundary move a finite distance away in finite time. As a result, no matter how finely the front is discretised, it will invariably be necessary to regrid it and add new grid points near the top. Moreover the asymptotic analytical solution shows where these new grid points in the neighbourhood of the top boundary should be placed to capture the front shape accurately and to preserve stability of the numerical scheme.

The singular nature of the pressure-driven growth equations and of their solutions thus impacts directly on numerical schemes. Indeed one must be careful that a numerical scheme avoids introducing spurious kinks into a solution that should not contain them. Spurious kinks could develop, for instance, if truncation and/or round-off errors cause points on the front to fall behind their neighbours thereby introducing local concavities.

There are numerical strategies that prevent concavities from developing into problematic kinks. Introducing a small amount of 'diffusion', i.e. a small but finite surface tension, is one such strategy. The appropriate value of surface tension is set by the maximum permitted front curvature, which in turn is set by the finite thickness of a low mobility wet foam zone adjacent to the front. Numerical diffusion (i.e. replacing the motion of a point 
on the front by a weighted sum of its own original motion and that of its neighbours, as proposed by de Velde Harsenhorst et al. (2014)) has an analogous effect.

These 'diffusive strategies' tend however to involve small spatial steps and small time steps in numerical schemes, which can become expensive. A simple alternative is to return to a pure pressure-driven growth (i.e. without 'diffusion') but to formulate an evolution rule whereby concave regions propagate at a different apparent speed from convex ones.

Such an evolution rule is potentially very useful not only for preventing undesired spurious kinks, but also for propagating any 'desired' sharp corners which might be an inherent part of a pressure-driven growth system. Even though sharp corners were absent from the cases studied by of Shan \& Rossen (2004) and de Velde Harsenhorst et al. (2014), there are at least 3 simple generalizations of this solution (all of potential engineering importance) where corners can develop, at least assuming an idealised situation where the low mobility zone at the foam front is treated as infinitesimally thin. These concern the case of slumping of surfactant solution, an increase in driving pressure, and a stratified reservoir. Detailed solution of these systems, and the propagation of the sharp corners therein, is left for further work.

\section{Acknowledgements}

GM and SC acknowledge financial support from FP7 IAPP project HYDROFRAC (PIAP-GA-2009-251475). PG and WR also acknowledge support from FP7 IAPP project HYDROFRAC (PIAP-GA-2009-251475) which funded a visit to Aberystwyth. PG and SC acknowledge financial support from the Isaac Newton Institute for Mathematical Sciences to attend the programme 'Foams and Minimal Surfaces: 12 Years On', and acknowledge discussions with J. Taylor, J. Sethian and D. Weaire during that programme. EMH acknowledges scholarship funding from the EPS-CONACyT programme. Moreover part of this work was carried out whilst PG was a Royal Academy of Engineering/Leverhulme Trust Senior Research Fellow and funding from the fellowship is gratefully acknowledged. PG also acknowledges sabbatical stay funding from CONICET Argentina.

\section{Appendix A. Long-time behaviour of viscous froth model for foam front propagating through a reservoir}

In the main text we calculated the long-time asymptotic front shape for the case of pressure-driven growth. We indicated that this asymptotic shape exhibited a mild singularity whereby the tangent to the front meets the top surface of the reservoir at right angles, but the curvature diverges there. This then begs the question of what is the long-time asymptotic front shape when we regularise the system of equations by perturbing away from pressure-driven growth to a viscous froth system (equation (3.3)) with finite (but possibly quite weak) surface tension: this is the question addressed here.

The viscous froth system certainly cannot admit solutions with divergent curvature, as the front velocity would diverge under those circumstances. In fact the long-time behaviour of the viscous froth system is sensitive to precisely how we formulate the viscous froth model. We could set the surface tension parameter $\gamma^{\prime}$ in the viscous froth model to be some fixed (generally small) value. Alternatively, we could use equation (5.1) coupled to equation (2.12) which together imply that $\gamma^{\prime}$ be chosen as

$$
\gamma^{\prime}=\tau s^{\prime}
$$

where $s^{\prime}$ is the path length that the front has travelled and $\tau$ is a small parameter (representing the thickness of per unit path length travelled of a low mobility zone adjacent to 
the front). The rationale behind equation (A 1) is that as the low mobility zone's thickness grows over time, the maximum permitted front curvature should fall, and this can be achieved, within the framework of the viscous froth model, by increasing the strength of the (diffusive) surface tension term.

Equation (A 1) substituted into equation (3.3) leads to a front velocity $\mathrm{d} \boldsymbol{x}^{\prime} / \mathrm{d} t^{\prime}$

$$
\frac{\mathrm{d} \boldsymbol{x}^{\prime}}{\mathrm{d} t^{\prime}}=\left(\frac{\Delta P^{\prime}}{s^{\prime}}-\tau \kappa^{\prime}\right) \boldsymbol{n}
$$

where, as in the main text, $\Delta P^{\prime}$ is driving pressure difference (given by (2.11)), $\kappa^{\prime}$ is front curvature, and $\boldsymbol{n}$ is front normal.

We shall consider the long-time asymptotic state of this equation first, before returning to consider the long-time asymptotic behaviour of equation (3.3) with a fixed $\gamma^{\prime}$ later on.

\section{A.1. Viscous froth model with surface tension growing proportional to path length}

Equation (A 2) admits a steady state Young-Laplace solution with $\mathrm{d} \boldsymbol{x}^{\prime} / \mathrm{d} t^{\prime}=0$ and hence

$$
\kappa^{\prime}=\frac{\Delta P^{\prime}}{\tau s^{\prime}} .
$$

Here the net pressure difference $\Delta P^{\prime}$ depends solely on depth (see equation (2.11)) since it corresponds to the difference between the driving pressure and the local hydrostatic pressure. In view of this, equation (A 3) describes the shape of a pendant or sessile drop, a fact to which we have already alluded (within the main text).

In addition, in the long-time asymptotic state, provided the front has displaced a long distance from its original position, one can treat $s^{\prime}$ as being spatially uniform along the front. This is the same approximation utilised in Section 4 of the main text, and states that the differences in $s^{\prime}$ between different points on the front are insignificant compared to the total distance travelled by any given point. Thus we can treat $s^{\prime}$ as representing the distance travelled by, for example, the point located at the top of the reservoir.

The parameter $\tau$ we assume to be small (the ratio between the thickness of the low mobility zone at the front and the distance that the front itself has displaced must be small if we are to represent the front shape as a 1-D curve propagating in two dimensions): as we have already stated in the main text, small values of $\tau$ correspond to abrupt collapse of the foam as it dries out.

We have two boundary conditions similar to the conditions applied in Section 4 . We require that the front meets the top of the reservoir at right angles. We also require (supposing the total displacement of the front is large) that far behind the leading edge of the front, its orientation is horizontal (and it is located at unit depth below the top).

It is apparent that these two conditions can only be satisfied for a critical value of the product $\tau s^{\prime}$. The critical value turns out to be $\frac{1}{2}$ (a fact we will demonstrate shortly). Hence the final steady-state displacement of the foam front $s_{\text {steady }}^{\prime}$ satisfies

$$
s_{\text {steady }}^{\prime}=\frac{1}{2 \tau}
$$

Since $\tau$ is a small parameter, it is clear that $s_{\text {steady }}^{\prime}$, is a large quantity, so the foam front displaces through a large distance before eventually coming to rest. This coincides with our intuition. Since surface tension is inherently weak, the pressure-driven contribution to the front velocity in equation (A 2 ) needs to accumulate a large drag coefficient (i.e. a large value of $s^{\prime}$ ) before it will fall to the same level as the surface tension.

As Figure 13 makes apparent, for values of $s^{\prime}$ that are larger than $s_{\text {steady }}^{\prime}$, equation (A 3) predicts a front shape that curves too little near the top of the reservoir, then penetrates 
too deeply into the system, and curves back on itself looking like a pendant drop. Meanwhile for values of $s^{\prime}$ that are smaller than $s_{\text {steady }}^{\prime}$, equation (A 3) curves too sharply near the top of the reservoir, and does not penetrate deep enough, before turning upwards again: this solution looks like a sessile drop. Only the case $s^{\prime}=s_{\text {steady }}^{\prime}$ satisfies the required boundary conditions (as described above).

\section{A.1.1. Solution for final steady front shape}

Substituting equation (A 4) into (A 3) (and also substituting from (2.11)) we obtain

$$
-\frac{\mathrm{d}^{2} \xi / \mathrm{d} y^{\prime 2}}{\left(1+\left(\mathrm{d} \xi / \mathrm{d} y^{\prime}\right)^{2}\right)^{3 / 2}}=2 y^{\prime}
$$

where, as in the main text, $\xi$ is a shifted $x^{\prime}$ coordinate such that the top of the front is at $\xi=0$ and $y^{\prime}=1$. Equation (A 5) can then be integrated once to obtain

$$
\frac{\mathrm{d} \xi / \mathrm{d} y^{\prime}}{\left(1+\left(\mathrm{d} \xi / \mathrm{d} y^{\prime}\right)^{2}\right)^{1 / 2}}=1-y^{\prime 2}
$$

which rearranges to

$$
\frac{\mathrm{d} \xi}{\mathrm{d} y^{\prime}}=\frac{\left(1-y^{\prime 2}\right)}{y^{\prime} \sqrt{2-y^{\prime 2}}} .
$$

Note that this not only satisfies the required top boundary condition $\mathrm{d} \xi / \mathrm{d} y^{\prime} \rightarrow 0$ as $y^{\prime} \rightarrow 1$, but also satisfies $\mathrm{d} y^{\prime} / \mathrm{d} \xi \rightarrow 0$ as $y^{\prime} \rightarrow 0$ (proving that equation (A 4 ) is correct).

Integrating equation (A 7)

$$
\xi=-\int_{y^{\prime}}^{1} \frac{\left(1-\mathcal{Y}^{2}\right)}{\sqrt{2-\mathcal{Y}^{2}}} \frac{\mathrm{d} \mathcal{Y}}{\mathcal{Y}}
$$

where $\mathcal{Y}$ is a dummy integration variable. The upper limit of integration has been chosen to ensure that $\xi \rightarrow 0$ as $y^{\prime} \rightarrow 1$ (which is true by definition). Equation (A 8) evaluates to

$$
\xi=\sqrt{2-y^{\prime 2}}-\frac{1}{\sqrt{2}} \log \left(\frac{2^{3 / 2} \sqrt{2-y^{\prime 2}}}{y^{\prime}}+\frac{4}{y^{\prime}}\right)-1+\frac{1}{\sqrt{2}} \log \left(2^{3 / 2}(1+\sqrt{2})\right)
$$

which is plotted in Figure 13 (i.e. the case $\tau s^{\prime}=\frac{1}{2}$ ).

\section{A.1.2. Behaviours near the top and bottom of the front}

Explicit forms for $y^{\prime}$ as a function of $\xi$ are available in the limits either as $y^{\prime} \rightarrow 1$ or $y^{\prime} \rightarrow 0$. The asymptotic behaviour as $y^{\prime} \rightarrow 1$ is most easily obtained via equation (A 5) which approximates to

$$
\mathrm{d}^{2} \xi / \mathrm{d} y^{\prime 2} \sim-2
$$

from which we deduce $\xi \approx-\left(1-y^{\prime}\right)^{2}$ and hence (with $\xi \leqslant 0$ in the domain of interest)

$$
y^{\prime} \sim 1-\sqrt{-\xi}
$$

The asymptotic behaviour as $y^{\prime} \rightarrow 0$ can be obtained via equation (A 9) to conclude that $\xi \rightarrow-\infty$ and

$$
y^{\prime} \sim 2^{3 / 2}(1+\sqrt{2})^{-1} \exp (-(2-\sqrt{2}-\sqrt{2} \xi)) .
$$

\section{A.1.3. Comparison between computed front shapes}

Note that there are superficial similarities between (steady state, Young-Laplace) equations (A 5)-(A 12) above and (pressure-driven growth) equations (4.8)-(4.15) in the main 
text. Both sets of equations describe functions that approach $y^{\prime} \rightarrow 1$ as $\xi \rightarrow 0$ (with in addition, $\mathrm{d} \xi / \mathrm{d} y^{\prime} \rightarrow 0$ there). Likewise both sets of equations describe functions that approach $y^{\prime} \rightarrow 0$ as $\xi \rightarrow-\infty$.

However there are subtle mathematical differences. Equations (4.13)-(4.15) indicate infinite curvature as $y^{\prime} \rightarrow 1$, whereas equations (A 10)-(A 11) indicate finite curvature there (which is unsurprising given that they ultimately come from a Young-Laplace law). Likewise the rate of approach to the limiting value $y^{\prime} \rightarrow 0$ for large and negative $\xi$ differs between equation (4.12) and equation (A 12).

More importantly though the physics is rather different. Equations (4.8)-(4.15) describe a propagating foam front driven by pressure and retarded by viscous drag, i.e. the case of pressure-driven growth: the shape of the propagating front is fixed at long times. Meanwhile equations (A 5)-(A 12) describe a steady foam front with pressure and surface tension in balance, with no propagation. The surface tension starts off very weak (i.e. the parameter $\tau$ is small), but the strength of the tension increases with distance travelled (modelling the fact that the thickness of the low mobility zone at the foam front gradually increases, making it increasingly difficult to bend it into very tight curvatures): an eventual balance with the driving pressure is inevitable. Given the smallness of the parameter $\tau$ however, balance is achieved only at very large $s_{\text {steady }}^{\prime}$, whereas, at much shorter times, long before steady state is achieved (i.e. for $s^{\prime} \ll s_{\text {steady }}^{\prime}$ ), surface tension makes instead a negligible contribution to the evolution of the viscous froth (provided the parameter $\tau$ is small).

Thus the early-time behaviour of the pressure-driven growth and viscous froth systems are similar (both qualitatively and quantitatively), whereas clearly their long-time behaviours are quite different (even qualitatively). This then begs the question of whether it is possible to consider an alternative viscous froth system which manages somehow to be a 'smoothed' version of pressure-driven growth, but which retains similar behaviours to pressure-driven growth at all times. Such a system is considered in the next subsection.

\section{A.2. Viscous froth model in the case of fixed weak surface tension}

We saw in the previous subsection that a balance between driving pressure and surface tension became possible, if the surface tension started out weak but then grew in strength over time. This stopped propagation of the foam front, meaning that viscous drag forces (which are only active when the foam front propagates) also ceased.

If we wish instead to formulate the viscous froth model so as to retain, even out to very long times, a dominant balance between pressure and viscous drag (as in pressure-driven growth) but with just a weak perturbation from surface tension, then it is clear that we should simply hold $\gamma^{\prime}$ in equation (3.3) to be a fixed small value.

It is equally clear however that we are dealing with a singular perturbation here. The formal $\gamma^{\prime} \rightarrow 0$ limit of equation (3.3) is equation (2.10), which at long times has a solution given by equation (4.9)-(4.10). This has curvature $\kappa^{\prime}$ diverging at the top of the reservoir, contradicting the notion that the surface tension term $\gamma^{\prime} \kappa^{\prime}$ in equation (3.3) is negligibly small. We must therefore consider a small region near the top of the system where surface tension effects are retained, and match this onto the pressure-driven growth solution (equations (4.9)-(4.10)) lower down. We must also determine whether the existence of small but finite surface tension affects the speed at which the front propagates.

These then are the issues that we address in this section. 


\section{A.2.1. Derivation of governing equation for a propagating foam front}

We begin our analysis by projecting equation (3.3) along the normal to obtain

$$
u^{\prime}=\frac{\Delta P^{\prime}-\gamma^{\prime} \kappa^{\prime}}{s^{\prime}}
$$

where $u^{\prime}$ is the speed of a material point. If the entire structure appears to propagate horizontally with an apparent speed $u_{a p p}^{\prime}$, then

$$
u_{\text {app }}^{\prime}=\frac{u^{\prime}}{\sin \alpha_{c}}
$$

where $\alpha_{c}$ is the angle the front tangent makes with the horizontal (see Figure 2(b)).

Near the top boundary, we are interested in values of $\alpha_{c}$ which are close to $\frac{\pi}{2}$. Equation (A 14) can then be approximated by

$$
u^{\prime} \approx u_{a p p}^{\prime}\left(1-\frac{1}{2}\left(\frac{\pi}{2}-\alpha_{c}\right)^{2}\right) .
$$

The value of $\Delta P^{\prime}$ is given by equation (2.11) where $y^{\prime}$ in that equation is a vertical coordinate. However near the top boundary the front tangent is nearly vertical, and hence

$$
\Delta P^{\prime} \approx 1-S^{\prime}
$$

where $S^{\prime}$ is distance measured along the (locally) near-vertical front, down from the top.

Finally (by definition)

$$
\kappa^{\prime}=-\frac{\mathrm{d} \alpha_{c}}{\mathrm{~d} S^{\prime}}
$$

Note the sign in the definition here, because $\alpha_{c}$ should fall as $S^{\prime}$ increases moving deeper into the reservoir.

Substituting equations (A 15)-(A 17) into equation (A 13), we obtain

$$
1-S^{\prime}+\gamma^{\prime} \frac{\mathrm{d} \alpha_{c}}{\mathrm{~d} S^{\prime}} \approx s^{\prime} u_{a p p}^{\prime}\left(1-\frac{1}{2}\left(\frac{\pi}{2}-\alpha_{c}\right)^{2}\right) \text {. }
$$

The value of $u_{a p p}^{\prime}$ is a priori unknown, but by definition is the speed of the point at the top of the reservoir, as the front tangent is vertical there. Note also that (by definition)

$$
u_{a p p}^{\prime}=\frac{\mathrm{d} s^{\prime}}{\mathrm{d} t^{\prime}}
$$

since the speed of that top boundary point is also the rate of change of the distance which that point has travelled. Moreover in the current limit (of long times and long distances travelled) we expect that $s^{\prime}$ is spatially uniform (and only varies with time).

For any given surface tension $\gamma^{\prime}$, we do not know a priori the value of the product $s^{\prime} u_{a p p}^{\prime}$. In the formal limit $\gamma^{\prime} \rightarrow 0$, we know (via equations (2.17) and (A 19)) that $s^{\prime} u_{a p p}^{\prime} \rightarrow 1$, neglecting the exceedingly small parameter $s_{0}^{\prime}$ in equation (2.17) in the long-time limit. For small but finite $\gamma^{\prime}$ we postulate (see also note $(w)$ ) that

$$
s^{\prime} u_{a p p}^{\prime}=1-\delta
$$

where $\delta$ is a small parameter to be determined. As equation (A 19) still applies, we deduce

$$
s^{\prime}=\sqrt{2(1-\delta) t^{\prime}} .
$$

Writing equation (A 18) in terms of angle $\alpha$ (the complement of $\alpha_{c}$, see Figure 2(b)), 
and retaining only leading order terms, we deduce

$$
\gamma^{\prime} \frac{\mathrm{d} \alpha}{\mathrm{d} S^{\prime}}=-S^{\prime}+\frac{1}{2} \alpha^{2}+\delta
$$

This then is the governing equation that we seek, for the singular perturbation region near the top of the propagating foam front. Analysis of this governing equation is performed in the next subsection.

\section{A.2.2. Analysis of governing equation for a propagating foam front}

Note that we expect the curvature term $\mathrm{d} \alpha / \mathrm{d} S^{\prime}$ in equation (A 22) to be positive, since $\alpha$ increases moving deeper into the reservoir (see Figure 2(b)). However according to equation (A 22) there are three terms (on the right hand side of the equation) contributing to the curvature. The physical interpretation of these three terms is as follows.

The first term $\left(-S^{\prime}\right)$ is a negative term and corresponds to a Young-Laplace effect. As we move deeper into the reservoir, the net hydrodynamic driving pressure decays, so the foam front becomes less curved, i.e. the perturbation to curvature is negative: we have already studied this Young-Laplace system in detail in Section A.1.

The second term $\left(\frac{1}{2} \alpha^{2}\right)$ is a positive term, and corresponds to a "curvature boundary layer' effect already studied extensively by Grassia et al. (2008a). Moving deeper into the system, as the normal to the front reorients (and deviates increasingly from the horizontal) its curvature must increase. The reason behind this curvature increase is that (owing to the front reorientation) the same apparent propagation speed can be obtained with a lesser speed of material points.

The third term $(\delta)$ represents the fact that a decrease in apparent velocity correlates with an increase in curvature.

As we move away from the top boundary these three terms on the right hand side must quickly come into balance. Otherwise (given that $\gamma^{\prime}$ on the left hand side is small), excessively large changes in the value of $\alpha^{\prime}$ with respect to changes in $S^{\prime}$ will result.

There are two branches for $\alpha$ achieving the required balance

$$
\alpha \approx \pm \sqrt{2\left(S^{\prime}-\delta\right)}
$$

As $S^{\prime}$ increases moving deeper and deeper into the system, the term $S^{\prime}$ in equation (A 23) must become larger than the constant $\delta$. Thus equation (A 23) becomes

$$
\alpha \approx \pm \sqrt{2 S^{\prime}}
$$

This then suggests that (even though the first, second and third terms on the right hand side of equation (A 22) must ultimately balance), the leading order balance is actually between the first and second terms.

We expect moreover, as alluded to above and as in Figure 2, that the point on the top boundary will displace the furthest, with points below that displacing slightly less: this requires positive values of $\alpha$, and hence

$$
\alpha \approx \sqrt{2 S^{\prime}}
$$

Indeed this follows directly from equation (4.14), where for small values of $\alpha$ we identify $\mathrm{d} \xi / \mathrm{d} y^{\prime}$ in that equation with $\alpha$, and identify $1-y^{\prime}$ with $S^{\prime}$.

Equation (A 22) (with leading order asymptotic solution equation (A 25)) then matches an inner region near the top boundary where surface tension effects are significant, with an outer region (equations (4.9)-(4.10)) far from the top boundary where they are not. 


\section{A.2.3. Consideration of balances within governing equation}

Even though we have stated that the first and second terms on the right hand side of equation (A 22) should eventually balance at leading order, it is instructive to consider hypothetical cases where one or other term dominates.

In the hypothetical case where the first term is assumed dominant, the solution is

$$
\alpha \sim-\frac{S^{\prime 2}}{2 \gamma^{\prime}},
$$

where recall that by assumption $\gamma^{\prime} \ll 1$. If this were taken to be the solution, it is clear that the (neglected) second term on the right hand side of equation (A 22) would become comparable with the first as soon as $S^{\prime}$ becomes comparable with $O\left(\gamma^{2 / 3}\right)$, i.e. as soon as $\alpha$ becomes $O\left(\gamma^{1 / 3}\right)$.

In the hypothetical case where the second term (on the right hand side of equation (A 22)) is assumed to dominate the first, a solution is (still for the case $\gamma^{\prime} \ll 1$ )

$$
\alpha \sim \frac{2 \gamma^{\prime}}{\left(S_{B L}^{\prime}-S^{\prime}\right)}
$$

where $S_{B L}^{\prime}$ denotes the extent of a 'curvature boundary layer' (i.e. the particular value of $S^{\prime}$ at which $\alpha$ would diverge, although note that strictly speaking equation (A 15), on the basis of which equation (A 22) was derived, breaks down in that limit). Again it is apparent (via equation (A 27)) that if $S^{\prime}$ moves within a distance $O\left(\gamma^{\prime 2 / 3}\right)$ of $S_{B L}^{\prime}$, then $\alpha$ becomes $O\left(\gamma^{1 / 3}\right)$.

\section{A.2.4. Rescaling the governing equation for a propagating foam front}

Our above analysis of equations (A 26)-(A 27) suggests introducing rescalings as follows

$$
S^{\prime}=\gamma^{\prime 2 / 3} r, \quad \alpha=\gamma^{1 / 3} A, \quad \delta=\gamma^{2 / 3} \Delta,
$$

where $r$ is rescaled distance (measured along the front), $A$ is rescaled angle, and $\Delta$ is an (a priori unknown) rescaled perturbation to the apparent propagation velocity. These scalings imply that the inner region, where surface tension terms are retained, extends a distance $O\left(\gamma^{\prime 2 / 3}\right)$ down from the top, films reorient by $O\left(\gamma^{\prime 1 / 3}\right)$ angles and curvatures are $O\left(1 / \gamma^{\prime 1 / 3}\right)$ there, but the resulting perturbation in the front velocity is only $O\left(\gamma^{\prime 2 / 3}\right)$.

It follows from equation (A 22) that

$$
\frac{\mathrm{d} A}{\mathrm{~d} r}=-r+\frac{1}{2} A^{2}+\Delta .
$$

Our rescaling ensures that (for $r$ and $\Delta$ values of order unity) all four terms in equation (A 29) can be of the same order of magnitude.

The boundary condition at the top of the reservoir demands $A=0$ when $r=0$. Equation (A 29) is then solved via a standard Runge-Kutta technique given any $\Delta$. Our task however is to find the particular value of $\Delta$ such that asymptotic boundary condition (A 24) is satisfied in the limit of large $r$ : this corresponds to a shooting technique.

\section{A.2.5. Analysis of rescaled governing equation}

The results of the shooting technique applied to equation (A 29) for various values of $\Delta$ are shown in Figure 14. If $\Delta$ is chosen too large, then $A$ grows too quickly, and (as $r$ increases) $\frac{1}{2} A^{2}$ exceeds $r$ : this leads to catastrophic divergence of $A$ (similar to what equation (A 27) predicts). Likewise if $\Delta$ is chosen too small, then $A$ grows slowly initially, the term in $-r$ begins to dominate (on the right hand side of equation (A 29)), quickly leading to negative values of $A$. Eventually $A$ reaches the neighbourhood of $-\sqrt{2(r-\Delta)}$ 
and thereafter continues to follow this negative solution branch. It is possible to perform a perturbation analysis about this solution branch, to demonstrate that solutions of equation (A 29) converge stably to it. We do not present the analysis here, but merely note that the fact that the converged value $-\sqrt{2(r-\Delta)}$ is variable rather than constant presents some (minor) complications in the perturbation analysis. This negative branch is not however the one we seek: we require a positive solution branch (see equation (A 25)).

As is clear from Figure 14, for the correctly chosen value of $\Delta$ (which we find to be between 1.283598 and 1.283599), solutions can be made to follow the positive solution branch. However if the choice for $\Delta$ is very slightly incorrect, the solutions follow the positive solution branch for a reasonable distance, and then either diverge to infinity or migrate across to the negative solution branch. A perturbation analysis about this positive branch reveals that it is 'unstable'. Note however that we use the term 'unstable' in a very specific context here, i.e. in the context of solving equation (A 29) to deduce the shape of a steadily propagating front. Were we to take a front shape close to the steadily propagating shape, and solve the unsteady state viscous froth equations, it is still reasonable to expect that the shape evolves stably towards the correct steady solution.

The $A$ vs $r$ formula can now be deduced via the numerical solution with the correct $\Delta$ value taken up to about $r \approx 6$ (the numerical solutions in Figure 15 are then still visibly extremely close to the desired branch), and thereafter using an analytic approximation

$$
A \approx \sqrt{2(r-\Delta)}
$$

Equation (A 30) is visibly closer to the numerical solutions than the slightly less accurate approximation $A \sim \sqrt{2 r}$, but further improvements are still possible, e.g. by substituting equation (A 30) into the left hand side of (A 29), and rearranging to obtain

$$
A \approx \sqrt{2(r-\Delta)+\frac{2}{2(r-\Delta)}} .
$$

This is extremely close to the numerical solution, except in the domain $r \lesssim 2$ (where it is not in any case designed to apply).

\section{A.2.6. Prediction of viscous froth front shapes near the top of the solution domain}

The numerical solutions of equation (A 29) and/or the analytical approximations (A 30) or (A 31), only supply information about angular orientation of the front vs distance measured along it.

In order to convert to a set of coordinate locations on the front we need to perform an additional integration. Near the top of the system, distances measured along the front $\left(S^{\prime}\right.$ and its rescaled analogue $r$ ) are almost vertical, whilst angular orientations ( $\alpha$ and its rescaled analogue $A$ ) measure deviations of the front tangent vector from the horizontal.

We deduce that the horizontal coordinate $\xi$ (relative to the leading edge at the top of the foam front) satisfies

$$
\xi=-\int_{0}^{S^{\prime}} \alpha \mathrm{d} \mathcal{S}
$$

where $\mathcal{S}$ is a dummy integration variable.

If we define a rescaled horizontal coordinate $\Xi$ by

$$
\xi=\gamma^{\prime} \Xi
$$


it follows from equations (A 28), (A 32) and (A 33) that

$$
\Xi=-\int_{0}^{r} A \mathrm{~d} r^{\prime}
$$

where $r^{\prime}$ is a dummy integration variable.

We can compute $\Xi$ by using the numerical solution of (A 29) for $A$ as long as it tracks the required solution branch, and then switch to integrating the analytical approximations (A 30) or (A 31) once the numerical solution deviates away from the required branch.

The result is shown in Figure 16. It is convenient to plot $-r$ vs $\Xi$ since this has the same orientation near the top boundary as a plot of $y^{\prime}$ vs $x^{\prime}$ as in Figure 5.

We can compare the $-r$ vs $\Xi$ shape computed above for a viscous froth, with the analogous shape near the top boundary for pressure-driven growth derived via equation (4.13)

$$
-\Xi=\frac{2 \sqrt{2}}{3} r^{3 / 2}
$$

It is clear from Figure 16 that the pressure-driven growth case curves more sharply than the viscous froth (see note $(x)$ ), which is unsurprising given we already know for pressuredriven growth that curvature diverges at the top. For the viscous froth case, curvature must however be finite at the top (otherwise the front would recede, not advance).

Equating $\mathrm{d} \boldsymbol{x}^{\prime} / \mathrm{d} t^{\prime}$ (at the top boundary) from equation (3.3) with $u_{a p p}^{\prime}$ from equation (A 19), and substituting from (A 20), reveals that for the viscous froth the curvature at the top boundary (denoted $\kappa_{\text {top }}^{\prime}$ ) is (see also note $(y)$ ):

$$
\kappa_{\text {top }}^{\prime}=\left(\gamma^{\prime}\right)^{-1 / 3} \Delta
$$

So in the viscous froth case, the curvature is large (but still finite) when $\gamma^{\prime}$ is small, but the effect on the apparent propagation speed scales as $\gamma^{\prime} \kappa_{\text {top }}^{\prime}$ and so remains small.

\section{A.2.7. Summary: Viscous froth model with weak surface tension}

In summary, the long-time behaviour of a viscous froth system (when utilised to describe the propagation of a foam front through a reservoir) depends on exactly how the viscous froth system is set up. If the surface tension in the viscous froth model is considered to start off very weak, but then gradually grows with distance travelled, then, after very long times and after propagating very long distances, the front is predicted eventually to come to rest. On the other hand, if the surface tension in the viscous froth model is held at a fixed but very weak level, then the foam front can propagate indefinitely. Either of the above two viscous froth models could be used to 'regularise' the behaviour of the pressure-driven growth system (which has identically zero surface tension) if one is interested in propagating the system only out to some specified finite time.

However the pressure-driven growth model predicts a front propagating indefinitely at long times. Thus only the latter type of viscous froth model (i.e. with fixed but weak surface tension) has the same qualitative long-time behaviour as pressure-driven growth. The viscous froth model is however a singular perturbation of the pressure-driven growth model: careful analysis of the solutions is required to match high curvature regions near the top of the reservoir (where surface tension effects retain moderate importance) to less curved regions lower down (where surface tension effects can be neglected). 


\section{Appendix B. Spurious formation of loops (\& mechanisms to avoid it)}

In the main text we found that (according to numerical predictions in Figure 6) kink formation on a foam front is followed by formation of topologically infeasible loops. Here we explain how this spurious numerical behaviour comes about (and how to avoid it).

We consider a geometry similar to that already considered in Figure 9. Specifically we consider (see Figure 17(a)) three discrete points (labelled $\boldsymbol{x}_{n-1}^{\prime}, \boldsymbol{x}_{n}^{\prime}$ and $\boldsymbol{x}_{n+1}^{\prime}$ ) each separated by a distance $\delta S^{\prime}$ and the front turns through an angle $\delta \theta / 2$ either side of $\boldsymbol{x}_{n}^{\prime}$, giving a total turn through $\delta \theta$.

Suppose we were to propagate points $\boldsymbol{x}_{n-1}^{\prime}, \boldsymbol{x}_{n}^{\prime}$ and $\boldsymbol{x}_{n+1}^{\prime}$ as material points, and suppose further that material points propagate at a speed $u^{\prime}$. For simplicity we assume for the purposes of Figure 17 that the speed of all material points is the same (which is a valid assumption provided the separation distance $\delta S^{\prime}$ is small).

After a time $\left(\delta S^{\prime} \cot (\delta \theta / 2)\right) / u^{\prime}$, corresponding to a displacement $\left(\delta S^{\prime} \cot (\delta \theta / 2)\right)$, material points $\boldsymbol{x}_{n-1}^{\prime}$ and $\boldsymbol{x}_{n+1}^{\prime}$ have become coincident, whilst material point $\boldsymbol{x}_{n}^{\prime}$ lags behind them by an amount $\delta S^{\prime}(1-\cos (\delta \theta / 2)) / \sin (\delta \theta / 2)$ : see Figure $17(\mathrm{~b})$. Since the edge joining $\boldsymbol{x}_{n-1}^{\prime}$ to $\boldsymbol{x}_{n}^{\prime}$ is now (essentially) parallel to that joining $\boldsymbol{x}_{n}^{\prime}$ to $\boldsymbol{x}_{n+1}^{\prime}$ (the residual angle between these near parallel edges has been exaggerated in the figure for clarity), any subsequent evolution would cause $\boldsymbol{x}_{n-1}^{\prime}$ and $\boldsymbol{x}_{n+1}^{\prime}$ to cross over one another and form a loop: see Figure 17 (c). Thus the spurious loop appearing in the numerical calculations in e.g. Figure 6 is associated with retaining material points such as $\boldsymbol{x}_{n}^{\prime}$ which strictly speaking should have been consumed by the evolution and removed.

The spurious behaviour can be avoided by propagating $\boldsymbol{x}_{n}^{\prime}$ not as a material point (with speed $\left.u^{\prime}\right)$ but instead as a geometric sharp corner with speed $u^{\prime} / \cos (\delta \theta / 2)$ : this was what was proposed in equations (5.8)-(5.9). After a time $\left(\delta S^{\prime} \cot (\delta \theta / 2)\right) / u^{\prime}$, the point will not lag behind $\boldsymbol{x}_{n-1}^{\prime}$ and $\boldsymbol{x}_{n+1}^{\prime}$ but instead catches up with (and indeed eventually becomes coincident with) them: see Figure $17(\mathrm{~d})$.

The edge joining $\boldsymbol{x}_{n-1}^{\prime}$ to $\boldsymbol{x}_{n}^{\prime}$ and that joining $\boldsymbol{x}_{n}^{\prime}$ to $\boldsymbol{x}_{n+1}^{\prime}$ are now both very short and (and being shorter than some minimum edge length of interest that would be set as a numerical parameter) could be eliminated from the calculation by e.g. discarding both points $\boldsymbol{x}_{n \pm 1}^{\prime}$, with the retained point $\boldsymbol{x}_{n}^{\prime}$ then being connected to its next nearest neighbours. The turning angle $\delta \theta$ at the retained point $\boldsymbol{x}_{n}^{\prime}$ is thereby preserved. Thus propagation via equation (5.8) or (5.9) should eliminate spurious loops.

Note that there could be slight differences in the evolution of the initial structure shown in Figure 17(a) - i.e. two straight edged segments initially turning through an angle $\delta \theta$ at a corner - according to whether one utilises equation (5.8) or equation (5.9).

We consider (as above, for simplicity), local behaviour in a sufficiently small region such that speed of material points $u^{\prime}$ is uniform. Equation (5.8) now predicts sensible behaviour, i.e. the entire structure preserves its shape and this shape propagates at an apparent speed $u_{a p p}^{\prime}=u^{\prime} / \cos (\delta \theta / 2)$.

Equation (5.9) predicts identical behaviour provided the initial turning angle $\delta \theta$ exceeds the model parameter $\delta \theta_{\text {sharp }}$ (in equation (5.9)): we expect this ordinarily to be the case, since we are free to select a $\delta \theta_{\text {sharp }}$ value much smaller than unity. If however $\delta \theta<\delta \theta_{\text {sharp }}$ (a fairly uncommon scenario when $\delta \theta_{\text {sharp }}$ is itself small), we have a rather severe numerical test of equation (5.9), since for any given numerical discretisation of the system, there is no a priori way of telling equation (5.9) that the location at which the two straight edges meet in Figure 17(a) really is intended to be treated as a corner (as opposed to a mere concavity). Thus that corner propagates initially as a material point. It is clear by comparing Figure 17(a)-(b) however, that (for a numerical interval spacing $\left.\delta S^{\prime}\right)$, propagating the structure in this way for a time $\delta S^{\prime} \cot (\delta \theta / 2) / u^{\prime}$ would lead to an 
arbitrarily sharp corner developing. Before that happens however, the turning angle at the corner begins to exceed the parameter $\delta \theta_{\text {sharp }}$. Then, according to equation (5.9), the corner will propagate at least as fast as $u^{\prime} / \cos \left(\delta \theta_{\text {sharp }} / 2\right)$. This is now faster than the apparent propagation speed of the straight sections of curve $u^{\prime} / \cos (\delta \theta / 2)$. The turning angle at the corner then decreases below $\delta \theta_{\text {sharp }}$ so the velocity of the corner falls back to $u^{\prime}$ again, and the behaviour repeats in an oscillatory fashion.

The precise nature of these oscillations in the velocity at the corner becomes sensitive to the space and time discretisation of the system (i.e. the chosen $\delta S^{\prime}$ and $\delta t^{\prime}$ ): it is only meaningful therefore to look at averaged system behaviour on much longer length and/or time scales. By oscillating about a turning angle $\delta \theta_{\text {sharp }}$, and spending part of the time with a velocity greater than $u^{\prime} / \cos (\delta \theta / 2)$ (and another part of the time with a velocity less than that), it is conceivable that the average velocity of the corner can match the apparent propagation velocity of the straight section of front. Thus in some time-averaged sense, equation (5.9) could match the predictions of equation (5.8). We reiterate however that we are dealing with a fairly severe numerical test of equation (5.9) here, and a relatively uncommon scenario. For suitably small choices of $\delta \theta_{\text {sharp }}$, equation (5.9) should for the most part immediately recognise corners with turning angle $\delta \theta$ and propagate them as such, without any numerical oscillations needing to set in.

\section{Appendix C. Correlations between permeability and foam mobility}

Section 6.3 and Figure 12 considered the case of a system stratified into high permeability and low permeability zones. Even the apparently simple statement that a front advances faster in a high permeability domain than in a low permeability domain, is far from obvious when one is dealing with non-Newtonian foam flows where there can be a correlation between permeability and mobility (Bertin et al. 1999; Yan et al. 2006). This has been the subject of a very recent study by Jones et al. (2013), and we summarise some of the key findings of that study here.

Permeability is most easily quantified via equation (2.1) in the case of a Newtonian fluid undergoing Poiseuille flow in a cylindrical pore, in which case it is possible to demonstrate that $k \propto R_{\text {pore }}^{2}$, where $R_{\text {pore }}$ is the cross-sectional pore radius.

Suppose we now consider a network of wide pores of radius $R_{\text {pore } 1}$ and a network of narrow pores of radius $R_{\text {pore } 2}$ (with $R_{\text {pore } 2}<R_{\text {pore } 1}$ ): the two networks are arranged in parallel with one another. We consider that the network lengths are equal and (since they are in parallel) that the pressure gradients along them are the same. Foam is now made to flow through these networks, and we assume that the individual bubble volumes are the same in both of them. Denote by $N_{1}$ and $N_{2}$ the number of films we encounter in a typical path traversing each network. On geometric grounds it is clear that

$$
N_{1}=\left(R_{\text {pore } 1}^{2} / R_{\text {pore } 2}^{2}\right) N_{2} \text {. }
$$

We also define the following notation: $\mu_{l i q}$ is the viscosity of the surfactant solution used to make the foam, $u_{\text {sup } i}$ is the superficial foam velocity in the pore (for $i=1$, wide pore, and $i=2$, narrow pore), and $\sigma$ is the tension of the film (we have deliberately not used the symbol $\gamma$ here, to avoid confusion with viscous froth equation (3.1) which employs surface tension in a rather different context). Moreover we define a capillary number as $C a_{i}=\mu_{l i q} u_{\text {sup } i} / \sigma$.

Literature on viscous dissipation in foams (Cantat et al. 2004), suggests that the pressure drop across the network $\Delta P$ should be proportional to $N_{i} C a_{i}^{m} \sigma / R_{\text {pore } i}$, where $m$ is a power law exponent (Bretherton 1961) normally taken to be $\frac{2}{3}$. 
Equating pressure drops implies

$$
N_{1} C a_{1}^{m} \sigma / R_{\text {pore } 1}=N_{2} C a_{2}^{m} \sigma / R_{\text {pore } 2},
$$

and hence (via equation $(\mathrm{C} 1)$ )

$$
C a_{1}^{m} / C a_{2}^{m}=R_{\text {pore } 2} / R_{\text {pore } 1} .
$$

It follows that the wide pore network has lower capillary number, and hence lower foam superficial velocity than the wide pore network. The reason for this (at first sight) paradoxical result is that the wide pore network has more films (and hence less mobility) than the narrow pore network.

The result in equation (C 3) is of course reliant on an assumption of equal bubble volumes in both the high and low permeability zones, which may not be relevant in a reservoir engineering context, where bubble size must be regulated by bubble generation and bubble coalescence events. Coalescence however is expected to be favoured in smaller pores (Bertin et al. 1998) which would tend to reduce $N_{2}$ relative to $N_{1}$, leading to a similar tendency, at least qualitatively, as equation (C 3).

If permeability in a network is modulated (via e.g. equation (6.5)), it is reasonable to suppose that certain parts of a foam front will run ahead of others. In that case, the arguments of Section 3 and Figure 12 (implying development of concavities and then sharp corners) still apply. However it may actually be the case that foam in low permeability zones runs ahead of that in high permeability zones (and not vice versa).

\section{Notes on the text}

(a) Specifically Shan \& Rossen (2004) took the quantity $S_{w}$ as introduced in equation (2.2) to be the average volume fraction of water throughout the foam zone.

(b) Specifically when switching from the 2-dimensional to the axisymmetric case, the formula for front shape $y^{\prime}$ vs $x^{\prime}$ at any given $t^{\prime}$ is unchanged (Shan \& Rossen 2004), but $x^{\prime}$ now denotes a radial coordinate, and the volume swept by foam is now given by $2 \pi \int_{0}^{x_{t o p}}\left(1-y^{\prime}\right) x^{\prime} \mathrm{d} x^{\prime}$ and the unswept volume is given by $2 \pi \int_{0}^{x_{t o p}} y^{\prime} x^{\prime} \mathrm{d} x^{\prime}$.

(c) In conventional formulations of viscous froth, the terms $k \lambda_{r} /\left(\left(1-S_{w}\right) \phi / \tau_{\text {thick }}\right)$ within equation (3.1) would normally be lumped together into a single parameter. We have not done so here however, in order to exploit better the analogy between viscous froth and pressure-driven growth.

(d) In equation (3.4) and the equations that follow, we deliberately adopt an 'unconventional' derivative notation here $\mathrm{d} / \mathrm{d} t^{\prime}$ and $\mathrm{d} / \mathrm{d} S^{\prime}$ instead of $\partial / \partial t^{\prime}$ and $\partial / \partial S^{\prime}$ (with $t^{\prime}$ being time and $S^{\prime}$ being arc length). This is intended to remind us that $\mathrm{d} / \mathrm{d} t^{\prime}$ is a time derivative following a given material element on the front, which differs from a time derivative at a given arc length, because material elements can shrink or stretch (Green et al. 2006; Satomi et al. 2013).

(e) Section 3.2 introduces 'sharp corners' and 'kinks'. Throughout we treat 'sharp corners' and 'kinks' as being similar, although we tend to utilise 'sharp corner' to represent an inherent geometric feature that may appear on a solution for the shape of a foam front, and 'kink' to represent an artifact that arises spuriously in a numerical solution.

(f) Although the Young-Laplace law conventionally considers pressure differences as being uniform along individual foam films, in the case of equation (2.11), which applies over much larger distance scales than a single bubble film, pressure is clearly non-uniform. The corresponding Young-Laplace law, equation (3.6), would then represent an overall energy minimum accounting for both surface and gravitational energies, subject to some given total volume of gas assumed to be present in the system. The analysis required 
would bear some similarities with computing the non-uniform curvature of a pendant or sessile drop (Huh \& Reed 1983; Loglio et al. 2003; Robertson \& Lehman 1968).

(g) Section 3.2 identified diffusivity of curvature as being the ratio between the surface tension $\gamma^{\prime}$ and the path length $s^{\prime}$. We assume path length $s^{\prime}$ to be an order unity quantity for the purposes of the simple order of magnitude arguments in Section 3.4.

(h) Section 3.5 discusses 'sharp corners' as being 'shocks' in the front shape, but recall that here we are considering an idealised situation: the low mobility wet foam zone that is found in the neighbourhood of the foam front, is treated as being infinitesimally thin, but in reality it should have a small but finite thickness. In this context a 'sharp corner' or 'shock' is an idealisation of the real foam front changing its tangent direction over a small length scale, comparable with the wet zone thickness.

(i) In Section 4.1, the only exception to the rule that values of $\sqrt{2 t^{\prime}}-x^{\prime}$ (the horizontal distances that points on the front fall short of the leading edge) are much smaller than $\sqrt{2 t^{\prime}}$ (the horizontal displacement of the leading edge itself) applies to vertical locations with $y^{\prime}$ values such that $y^{\prime} \ll 1$. By equation (2.11) these have very little net driving pressure difference, and so barely displace any further, even though points higher up in the reservoir are still displacing.

(j) Time $t^{\prime}=8$ considered in Figure 5(a) corresponds to a distance between the injection and production wells of $\sqrt{2 t^{\prime}}=4$ dimensionless units, corresponding to roughly $9 \mathrm{~km}$ dimensional distance in the case presented by de Velde Harsenhorst et al. (2014).

(k) It is possible to demonstrate that the early-time analytic solution for the front shape given by de Velde Harsenhorst et al. (2014) actually drives all points on the front downwards with vertical velocity equal to $\frac{1}{2}$. Thus, even at very small times, this solution is not valid uniformly over the entire solution domain, but instead only for $y^{\prime}<1-\frac{1}{2} t^{\prime}$. For $1-\frac{1}{2} t^{\prime}<y^{\prime}<1$ (still with $t^{\prime} \ll 1$ ), it must match some other solution. It is conceivable that, in the very top part of the solution domain, matching to a solution like equations (4.9)-(4.10) might be achieved even for small times $t^{\prime} \leqslant O(1)$, and then the extent of the vertical region over which that solution applies simply grows with time: this conjecture would be consistent with the data in Figure 5.

(l) The result of equation (4.11) is essentially unchanged regardless of whether we consider a 2-dimensional model geometry (as has been done here) or an axisymmetric geometry (in which case we are computing 'unswept volume' rather than 'unswept area'). The reason is that the unswept part of the system is localised in a small lateral region compared to the overall horizontal distance through which the front has propagated - so a locally Cartesian geometry can be employed. Specifically if $x_{t o p}$ denotes the dimensionless position of the leading edge of the foam front, the asymptotic unswept volume is simply the product of this unswept area $\frac{\pi}{4}$ and the front perimeter $2 \pi x_{t o p}$.

$(m)$ The implication in Section 4.2 that almost all vertical locations on the foam front are at most an order unity horizontal distance behind the leading edge is a notable difference from an alternative model presented by de Velde Harsenhorst et al. (2014) in which foam is assumed to propagate along but not downwards into a reservoir. In that case override is significant: by time $t^{\prime}$, points on the front at an arbitrary height $y^{\prime}$ fall behind the leading edge at the top typically by order $\sqrt{t^{\prime}}$ amounts.

$(n)$ Note that throughout Section 4, the long-time asymptotic analysis for the front shape is based on the premise that material points on the front have displaced further horizontally than vertically. Based on the analysis in Section 4.4.1 however, one can infer that the bulk of this large horizontal displacement was in fact incurred even before material points were injected onto the front from the top boundary.

(o) Although Section 4.4.1 alludes to the evolution of grid point positions (and the consequent need to add new grid points) specifically in the case of a singular curvature, 
in a numerical simulation it is not readily possible to distinguish a case where curvature is singular from one where curvature is finite but large. Adding new grid points will be necessary anywhere that both the front shape is convex and the front curvature is large.

$(p)$ Our numerical investigations indicate that it is better not to place a new grid point $y_{\text {fine }}$ midway between $y_{\text {top }}$ and $y_{\text {coarse }}$, but instead to place it closer to $y_{\text {top }}$. This gives the newly created edge (between $y_{\text {fine }}$ and $y_{\text {top }}$ ) more leeway to grow over time. At very early times, placing $y_{\text {fine }}$ too far from $y_{\text {top }}$ can also cause a spurious concavity to appear at $\left(x_{\text {coarse }}, y_{\text {coarse }}\right)$ : this spurious behaviour arises because equations (4.20)(4.21) assume sharp curvatures near the top boundary, but these are less well captured if $y_{\text {fine }}$ is placed too far from $y_{t o p}$.

(q) Deliberately introducing a concave defect close to the top boundary and at early time (as in Figure 6) represents a 'worst case scenario'. Were we to introduce a concave defect much lower down at a later time, the defect would then be a small vertical bump on an otherwise near horizontal section of front. Provided the near horizontal section is sufficiently close to the bottom of the foam front, it barely moves because the driving pressure difference across it is low. On the other hand, the bump itself, being vertically slightly higher up, experiences a slightly larger driving pressure difference, and so can still move downwards. In this fashion, the height of the bump could gradually diminish.

( $r$ ) A corollary of the observation of de Velde Harsenhorst et al. (2014), i.e. that a low level of noise introduced into a front shape takes a considerable time to develop into a problematic kink, is that one should endeavour to reduce the effects of noise arising from truncation error of the numerical scheme itself. The refinements mentioned in Section 5 (i.e. defining normal vectors as weighted averages of adjacent segments and/or using a Heun time stepping method) may help to reduce the noise due to spatial and/or temporal truncation error, keeping the system better behaved. Alternatively, given we noted in Section 3.5 that we are dealing with hyperbolic partial differential equations, standard numerical techniques for stabilising such systems (e.g. upwind spatial differences and/or implicit time-stepping (Press et al. 1992)) may be worth exploring. Indeed in Section 3.5 we noted the formal equivalence between the pressure-driven growth model and the eikonal equation, making it feasible to exploit existing numerical methods for the latter (see e.g. Saye \& Sethian (2011); Sethian (1996, 1999a,b); Sethian \& Vladimirsky (2000)).

(s) In the discussion of Section 5.1, in order to fix the location of the top boundary point, relative to nearest and next nearest neighbours, a parabolic shaped smooth curve could be fitted, notwithstanding the prediction of equation (4.13) which is patently not a parabola. Equation (4.13) however only applies to the singular case of pressure-driven growth, whereas any finite $\tau$ in equation (5.2) will relieve that singularity.

$(t)$ According to the numerical data utilising equation (5.8) as reported in Section 5.3, shrinking edge lengths appear to be accompanied by a gradual sharpening of the concavity (i.e. a gradual increase in the angle $\delta \theta$ ), even though equation (5.8) should in principle conserve $\delta \theta$. However conservation of $\delta \theta$ strictly only applies to the situation in Figure 9, which represents two perfectly straight sections of front each propagating at uniform speed, and turning through an angle $\delta \theta$ at a corner. The situation in our simulation is a little different, as we have a discretised representation of a curved film, and moreover there are non-uniformities in front speed (due to non-uniformities in driving pressure differences and in path lengths travelled): these features may explain the gradual sharpening observed. There is also the possibility that the gradual sharpening is partly an artifact of truncation error in the numerical scheme. We might anticipate significant truncation errors in the scheme when discrete segments have shrunk to the extent that the displacement of points on the front over a single numerical time step is 
comparable with the discrete segment length itself. Very short edges need to be removed if that situation occurs.

(u) The discussion of Section 6 refers extensively to 'sharp corners' on infinitesimally thin foam fronts. More realistically we would need to consider a finite thickness, low mobility wet zone in the neighbourhood of the foam front, and the analogue of a 'sharp corner' would be a significant change in the tangent to the front on a length scale comparable with the thickness of the low mobility zone (see also note $(h)$ earlier).

(v) Note that de Velde Harsenhorst et al. (2014) recently considered the case of a permeability that (owing to fine-scale stratification) is homogeneous but anisotropic. In Section 6.3 however we discuss the case of a permeability that is heterogeneous across strata but isotropic within each stratum. The case of heterogeneous and anisotropic permeability is an obvious extension of this system.

$(w)$ The postulate in equation (A 20) that curvature effects imply a reduction of propagation speed is reminiscent of the proposed effects of curvature in flame-propagation problems (Markstein 1951).

$(x)$ At first sight in Figure 16 the lesser curvature of the viscous froth system makes it appear that, moving deeper into the system, the viscous froth has advanced further and faster than the pressure-driven growth one. This is however a feature of plotting Figure 16 in terms of a horizontal coordinate $\Xi$ moving with the foam front, defined such that the leading edge of the front is always at $\Xi=0$. If one were to switch back to a fixed coordinate, the viscous froth curve would be seen to advance less than the pressure-driven growth curve as its propagation speed is lower.

(y) Although the formula (A 36) for $\kappa_{\text {top }}^{\prime}$ formally applies for a fixed $\gamma^{\prime}$ system out to arbitrary distances travelled $s^{\prime}$, if we invoke the criterion from Section 5.1 that curvature cannot exceed the reciprocal of the thickness $\tau_{\text {thick }}^{\prime}$, and if we assume via equation (2.12) that $\tau_{\text {thick }}^{\prime}=\tau s^{\prime}$ (where $\tau$ is a small parameter), then we deduce that $s^{\prime}$ should not exceed $\gamma^{\prime 1 / 3} /(\Delta \tau)$. However $\tau$ can be chosen arbitrarily small here.

\section{Nomenclature}

Roman symbols:

$A$ rescaled version of the angle $\alpha$

$C a_{1}$ capillary number for foam flow through a network of wide pores

$C a_{2}$ capillary number for foam flow through a network of narrow pores

$d_{\max }$ maximum depth to which foam can penetrate in the system

$D_{\text {curv }}$ (dimensionless) diffusivity of curvature

$\boldsymbol{e}_{x}$ horizontal unit vector

$\boldsymbol{e}_{y}$ vertical unit vector

$\boldsymbol{e}_{z}$ unit vector out of the plane

$g$ gravity

$k$ permeability

$k_{\text {strat }}$ relative amount that permeability is modulated (in a stratified reservoir)

$m$ a power law exponent

(relevant to foam flow through a network of pores)

$M_{\text {slump }}$ relative increase in foam mobility due to slumping

$n_{\text {strat }}$ number of maxima and minima of permeability (in a stratified reservoir)

$\boldsymbol{n}$ normal vector to foam front 
$N_{1}$ number of foam films encountered (whilst traversing a network of wide pores)

$N_{2}$ number of foam films encountered (whilst traversing a network of narrow pores)

$p_{f a c}$ a factor representing a relative increase in driving pressure

$\nabla P$ pressure gradient

$\Delta P$ pressure difference across front

$\Delta P^{\prime}$ dimensionless analogue of $\Delta P$

$P_{\text {drive }}$ driving pressure

$P_{h y d}$ hydrostatic pressure difference between liquid and gas

$r$ rescaled version of the arc length measured along a foam front $S^{\prime}$

$r_{\text {arc }}$ (dimensionless) radius of curvature of an arc on a front

$r_{\text {arc } 0}$ (dimensionless) initial radius of curvature of an arc on a front

$R_{\text {pore }}$ radius of cross-section of a pore

$s$ path length travelled by a material element

$s^{\prime}$ dimensionless analogue of $s$

$s_{0}^{\prime}$ initial value of $s^{\prime}$ (typically a small parameter)

$s_{\text {steady }}^{\prime}$ a large value of $s^{\prime}$ (at which a front may attain steady state)

$S$ arc length measured along foam front

$S^{\prime}$ dimensionless analogue of $S$

$S_{B L}^{\prime}$ extent of a curvature boundary layer (measured in terms of distance along a foam front)

$\delta S^{\prime}$ dimensionless grid spacing along foam front

$S_{w}$ volume fraction of water in the foam

$t$ time

$t^{\prime}$ dimensionless analogue of $t$

$\delta t^{\prime}$ a dimensionless time step

$t_{\text {scale }}$ characteristic time scale

$t_{\text {arb }}$ an arbitrary (dimensionless) time

$t_{\text {arc }}$ (dimensionless) time at which an arc is present on a front

$t_{\text {corner }}$ (dimensionless) time at which a sharp corner is present

$t_{\text {press }}$ (dimensionless) time at which driving pressure is increased

$t_{\text {slump }}$ (dimensionless) time at which slumping occurs

$\boldsymbol{t}$ tangent vector to foam front

$T$ (dimensionless) time elapsed since $t_{a r b}$

$\boldsymbol{u}_{\text {int }}$ interstitial front velocity

$\boldsymbol{u}_{\text {sup }}$ superficial front velocity

$u^{\prime}$ (dimensionless) speed of a front material point

$u_{\text {app }}^{\prime}$ apparent (dimensionless) speed of a sharp corner or kink (or, more generally, of any other structure that propagates)

$x$ horizontal coordinate

$x^{\prime}$ dimensionless analogue of $x$

$\boldsymbol{x}$ front position

$\boldsymbol{x}^{\prime}$ dimensional analogue of $\boldsymbol{x}$

$\boldsymbol{x}_{n}^{\prime}, \boldsymbol{x}_{n \pm 1}^{\prime}$ discrete grid points

$\delta \boldsymbol{x}_{\text {grid }}^{\prime}$ numerical grid spacing

$\left(x_{t o p}, y_{t o p}\right)$ a numerical grid point location at the top of the foam front $\left(x_{\text {coarse }}, y_{\text {coarse }}\right)$ a numerical grid point location below the top of the front (on a coarse grid) 
$\left(x_{\text {fine }}, y_{\text {fine }}\right)$ a numerical grid point location below the top of the front

(on a fine grid)

$y$ vertical coordinate

$y^{\prime}$ dimensionless analogue of $y$

$y_{\text {inst }}$ instantaneous (dimensionless) coordinate of a front material point

$y_{\text {slump }}$ (dimensionless) height to which a system slumps

Greek symbols:

$\alpha$ angle between front normal and horizontal (or equivalently between front tangent and vertical)

$\alpha_{c}$ complement of angle $\alpha$

$\gamma$ surface tension in the viscous froth model

$\gamma^{\prime}$ dimensionless analogue of $\gamma$ (a small parameter)

$\Gamma^{\prime}$ a weighting factor used to introduce numerical diffusivity (mathematically analogous to surface tension parameter $\gamma^{\prime}$ )

$\delta$ a small parameter measuring the velocity decrease of the foam front (due to surface tension effects)

$\Delta$ a rescaled value of the velocity decrease $\delta$

$\theta_{\text {incl }}$ included angle in a sharp corner

$\delta \theta$ angle through which film turns between successive grid elements

$\delta \theta_{\text {sharp }}$ turning angle between grid elements (deemed to correspond to a sharp corner)

$\kappa$ front curvature

$\kappa^{\prime}$ dimensionless analogue of $\kappa$

$\kappa_{\max }$ (dimensionless) maximum front curvature (scales inversely with thickness of a low mobility zone at the front)

$\kappa_{\text {top }}^{\prime}$ (dimensionless) front curvature at the top of the reservoir

$\lambda_{r}$ relative mobility

$\mu_{l i q}$ viscosity of surfactant solution used to make foam

$\xi$ dimensionless similarity variable for a propagating front (horizontal coordinate relative to the leading edge of the front)

$\Xi$ rescaled version of the horizontal coordinate $\xi$

$\rho$ density difference (between surfactant solution and gas)

$\sigma$ film tension for individual films flowing through a network of pores

$\tau_{\text {thick }}$ thickness of a low mobility zone at the foam front

$\tau_{\text {thick }}^{\prime}$ dimensionless analogue of $\tau_{\text {thick }}$

$\tau$ ratio of (low mobility zone) thickness to path length travelled (a small parameter)

$\phi$ porosity of reservoir

\section{REFERENCES}

Afsharpoor, A., Lee, G. S. \& Kam, S. I. 2010 Mechanistic simulation of continuous gas injection period during surfactant-alternating-gas (SAG) processes using foam catastrophe theory. Chem. Engng Sci. 65, 3615-3631.

Arnold, V. I. 2004 Lectures on Partial Differential Equations, 2nd edn. Berlin: Springer.

Ashoori, E., van der Heijden, T. L. M. \& Rossen, W. R. 2010 Fractional-flow theory of foam displacements with oil. SPE J. 15, 260-273.

Barry, J. D., Weaire, D. \& Hutzler, S. 2010 Shear localisation with 2D viscous froth and its relation to the continuum model. Rheol. Acta 49, 687-698. 
Bertin, H. J., Apaydin, O. G., Castanier, L. M. \& Kovscek, A. R. 1998 Foam flow in heterogeneous porous media: Effect of crossflow (Paper SPE 39678). In SPE/DOE Improved Oil Recovery Symposium, Tulsa, OK, 19th-22nd Apr..

Bertin, H. J., Apaydin, O. G., Castanier, L. M. \& Kovscek, A. R. 1999 Foam flow in heterogeneous porous media. J. Soc. Petroleum Engnrs 4, 75-82.

Blaker, T., Aarra, M. G., Skauge, A., Rasmussen, L., Celius, H. K., Martinsen, H. A. \& VAssenden, F. 2002 Foam for gas mobility control in the Snorre field: The FAWAG project. SPE Reservoir Evaluation \& Engg 5, 317-323.

Boeije, C. S. \& Rossen, W. R. 2014 Gas injection rate needed for SAG foam processes to overcome gravity override (Paper SPE 166232). Accepted for publication by SPE J. .

Brakke, K. A. 1978 The Motion of a Surface by its Mean Curvature. Princeton, NJ: Princeton University Press, available from page http://www.susqu.edu/brakke.

Bretherton, F. P. 1961 The motion of long bubbles in tubes. J. Fluid Mech. 10, 166-188.

Cantat, I., Cohen-Addad, S., Elias, F., Graner, F., Höhler, R., Pitois, O., Rouyer, F. \& Saint-Jalmes, A. 2013 Foams: Structure and Dynamics. Oxford: Oxford University Press.

Cantat, I., Kern, N. \& Delannay, R. 2004 Dissipation in foam flowing through narrow channels. Europhys. Lett. 65, 726-732.

Casademunt, J. \& Magdaleno, F. X. 2000 Dynamics and selection of fingering patterns. Recent developments in the Saffman-Taylor problem. Phys. Reports 337, 1-35.

Cottin, C., Bodiguel, H. \& Colin, A. 2010 a Drainage in two-dimensional porous media: From capillary fingering to viscous flow. Phys. Rev. E 82, 046315.

Cottin, C., Bodiguel, H. \& Colin, A. $2010 b$ Influence of wetting conditions on drainage in porous media: A microfluidic study. Phys. Rev. E 82, 026311.

Couder, Y., Cardoso, O., Dupuy, D., Tavernier, P. \& Thom, W. 1986 Dendritic growth in the Saffman-Taylor experiment. Europhys. Lett. 2, 237-443.

Cox, S. \& Mishuris, G. 2009 Remarks on the accuracy of algorithms for motion by mean curvature in bounded domains. J. Mechanics of Materials \& Structures 4, 1555-1572.

Cox, S., Weaire, D. \& Glazier, J. A. $2004 a$ The rheology of two-dimensional foams. Rheol. Acta 43, 442-448.

Cox, S. J. 2005 A viscous froth model for dry foams in the Surface Evolver. Colloids and Surf. A, Physicochem. and Engg Aspects 263, 81-89, a collection of papers presented at the 5th European Conference on Foams, Emulsions, and Applications, EUFOAM 2004, University of Marne-la-Vallee, Champs sur Marne (France), 5th-8th Jul. 2004.

Cox, S. J., Neethling, S., Rossen, W. R., Schleifenbaum, W., Schmidt-Wellenburg, P. \& Cilliers, J. J. $2004 b$ A theory of the effective yield stress of foam in porous media: The motion of a soap film traversing a three-dimensional pore. Colloids and Surf. A, Physicochem. and Engg Aspects 245, 143-151.

Cox, S. J., Weaire, D. \& Mishuris, G. 2009 The viscous froth model: Steady states and the high-velocity limit. Proc. Roy. Soc. London Ser. A 465, 2391-2405.

Drenckhan, W., Cox, S. J., Delaney, G., Holste, H., Weaire, D. \& Kern, N. 2005 Rheology of ordered foams - On the way to discrete microfluidics. Colloids and Surf. A, Physicochem. and Engg Aspects 263, 52-64.

Embley, B. \& Grassia, P. 2011 Viscous froth simulations with surfactant mass transfer and Marangoni effects: Deviations from Plateau's rules. Colloids and Surf. A, Physicochem. and Engg Aspects 382, 8-17.

Falls, A. H., Hirasaki, G. J., Patzek, T. W., Gauglitz, D. A., Miller, D. D. \& RatULOWSKI, T. 1988 Development of a mechanistic foam simulator: The population balance and generation by snap-off. SPE Reservoir Engineering 3, 884-892.

Fisher, A. W., Foulser, R. W. S. \& Goodyear, S. G. 1990 Mathematical modeling of foam flooding (Paper SPE/DOE 20195). In SPE/DOE Enhanced Oil Recovery Symposium, Tulsa, OK, 22nd-25th Apr..

Fullman, R. L. 1952 Boundary migration during grain growth. In Metal Interfaces, pp. 179207. American Society for Metals, Cleveland, Ohio.

Glazier, J. A. \& Weaire, D. 1992 The kinetics of cellular patterns. J. Phys.: Condens. Matter 4, 1867-1894. 
Goldstein, H. 1980 Classical Mechanics, 2nd edn. Reading, Mass.: Addison-Wesley Publishing Company.

Grassia, P., Montes-Atenas, G., Lue, L. \& Green, T. E. 2008 a A foam film propagating in a confined geometry: Analysis via the viscous froth model. Eur. Phys. J. E 25, 39-49.

Grassia, P., Usher, S. P. \& Scales, P. J. $2008 b$ A simplified parameter extraction technique using batch settling data to estimate suspension material properties in dewatering applications. Chem. Engng Sci. 63, 1971-1986.

Grassia, P., Usher, S. P. \& Scales, P. J. 2011 Closed-form solutions for batch settling height from model settling flux functions. Chem. Engng Sci. 66, 964-972.

Green, D. W. \& Willhite, G. P. 1998 Enhanced Oil Recovery. Richardson, TX: Society of Petroleum Engineers.

Green, T. E., Bramley, A., Lue, L. \& Grassia, P. 2006 Viscous froth lens. Phys. Rev. E 74, 051403.

Green, T. E., Grassia, P., Lue, L. \& Embley, B. 2009 Viscous froth model for a bubble staircase structure under rapid applied shear: An analysis of fast flowing foam. Colloids and Surf. A, Physicochem. and Engg Aspects 348, 49-58.

Haranczyk, M. \& Sethian, J. A. 2009 Navigating molecular worms inside chemical labyrinths. Proc. Nat. Acad. Sci. USA 106, 21472-21477.

HarAnczyk, M. \& Sethian, J. A. 2010 Automatic structure analysis in high-throughput characterization of porous materials. J. Chem. Theory Comput. 6, 3472-3480.

Hirasaki, G. J., Miller, C. A. \& Puerto, M. 2011 Recent advances in surfactant EOR. SPE J. 16, 889-907.

Hoefner, M. L. \& Evans, E. M. $1995 \mathrm{CO}_{2}$ foam: Results from four developmental fields trials. SPE Reservoir Evaluation \& Engg 10, 273-281.

Huh, C. \& Reed, R. L. 1983 A method for estimating interfacial tensions and contact angles from sessile and pendant drop shapes. J. Colloid and Interf. Sci. 91, 472-484.

Jones, S. A., Dollet, B., Méheust, Y., Cox, S. J. \& Cantat, I. 2013 Structure-dependent mobility of a dry aqueous foam flowing along two parallel channels. Phys. Fluids 25, 063101.

KAM, S. I. 2008 Improved mechanistic foam simulation with foam catastrophe theory. Colloids and Surf. A, Physicochem. and Engg Aspects 318, 62-77.

Kern, N. \& Weaire, D. 2003 Approaching the dry limit in foam. Phil. Mag. 83, 2973-2987.

Kern, N., Weaire, D., Martin, A., Hutzler, S. \& Cox, S. J. 2004 Two-dimensional viscous froth model for foam dynamics. Phys. Rev. E 70, 041411.

Kessler, D. A. \& Levine, H. $1986 a$ Theory of the Saffman-Taylor finger pattern I. Phys. Rev. A 33, 2621-2633.

Kessler, D. A. \& Levine, H. $1986 b$ Theory of the Saffman-Taylor finger pattern II. Phys. Rev. A 33, 2634-2639.

Kнatib, Z. I., Hirasaki, G. J. \& Falls, A. H. 1988 Effects of capillary pressure on coalescence and phase mobilities in foams flowing through porous media. SPE Reservoir Engineering 3, 919-926.

Knight, R. D. 2008 Physics for Scientists and Engineers with Modern Physics: A Strategic Approach, 2nd edn. San Francisco, CA: Pearson Addison-Wesley, see Chapter 22. Wave Optics.

Kovscek, A. R. \& Bertin, H. J. 2003 a Foam mobility in heterogeneous porous media. I. Scaling concepts. Transport in Porous Media 52, 17-35.

Kovscek, A. R. \& Bertin, H. J. $2003 b$ Foam mobility in heterogeneous porous media. II. Experimental observations. Transport in Porous Media 52, 37-49.

Kovscek, A. R., Patzek, T. W. \& Radke, C. J. 1995 A mechanistic population balance model for transient and steady-state foam flow in Boise sandstone. Chem. Engng Sci. 50, 3783-3799.

Kovscek, A. R. \& Radke, C. J. 1994 Fundamentals of foam transport in porous media. In Foams: Fundamentals and Applications in the Petroleum Industry (ed. L. L. Schramm), Advances in Chemistry, vol. 242, chap. 3, pp. 115-163. Washington, DC: American Chemical Society.

Kraynik, A. M. 1988 Foam flows. Ann. Rev. Fluid Mech. 20, 325-357.

Kynch, G. J. 1952 A theory of sedimentation. Trans. Faraday Soc. 48, 166-176.

LAKe, L. W. 2010 Enhanced Oil Recovery. Englewood Cliffs, NJ: Prentice Hall. 
Lester, D. R., Usher, S. P. \& Scales, P. J. 2005 Estimation of the hindered settling function $R(\phi)$ from batch-settling tests. AIChE J. 51, 1158-1168.

Li, R. F., Yan, W., Liu, S. H., Hirasaki, G. J. \& Miller, C. A. 2010 Foam mobility control for surfactant enhanced oil recovery. SPE J. 15, 934-948.

Lighthill, M. J. \& Whitham, G. B. 1955 On kinematic waves. II. A theory of traffic flow on long crowded roads. Proc. Roy. Soc. London Ser. A 229, 317-345.

Loglio, G., Pandolfini, P., Makievski, A. V. \& Miller, R. 2003 Calibration parameters of the pendant drop tensiometer: Assessment of accuracy. J. Colloid and Interf. Sci. 265, $161-165$.

Ma, K., Liontas, R., Conn, C., Hirasaki, G. J. \& Biswal, S. L. 2012 Visualization of improved sweep with foam in heterogeneous porous media using microfluidics. Soft Matter 8, 10669-10675.

Ma, K., Lopez-Salinas, J. L., Puerto, M. C., Miller, C. A., Biswal, S. L. \& Hirasaki, G. J. 2013 Estimation of parameters for the simulation of foam flow through porous media. Part 1: The dry-out effect. Energy \&6 Fuels 27, 2363-2375.

Markstein, G. H. 1951 Experimental and theoretical studies of flame-front stability. J. Aeronaut. Sci. 18, 199-209.

Martinsen, H. A. \& VAssenden, F. 1999 Foam-assisted water alternating gas (FAWAG) process at Snorre. In European IOR Symposium, Brighton, UK, 18th-20th Aug..

Moore, M. G., Juel, A., Burgess, J. M., McCormick, W. D. \& Swinney, H. L. 2002 Fluctuations in viscous fingering. Phys. Rev. E 65, 030601.

Mullins, W. W. 1956 Two-dimensional growth of idealized grain boundaries. J. Appl. Phys. 27, 900-904.

Peleg, A., Meerson, B., Vilenkin, A. \& Conti, M. 2001 Area-preserving dynamics of a long slender finger by curvature: A test case for globally conserved phase ordering. Phys. Rev. E 63, 066101.

Persoff, P., Radke, C. J., Pruess, K. \& Benson, S. M. 1991 A laboratory investigation of foam flow in sandstone at elevated pressure. SPE Reservoir Engg 6, 365-372.

PitTs, E. 1980 Penetration of fluid into a Hele-Shaw cell: Saffman-Taylor experiment. J. Fluid Mech. 97, 53-64.

Press, W. H., Teukolsky, S. A., Vetterling, W. T. \& Flannery, B. P. 1992 Numerical Recipes in C: The Art of Scientific Computing, 2nd edn. Cambridge: CUP, see Chapter 19. Partial Differential Equations.

Renardy, M. \& Rogers, R. C. 2004 An Introduction to Partial Differential Equations, 2nd edn., Texts in Applied Mathematics, vol. 13. New York, Berlin, Heidelberg: Springer-Verlag.

Robertson, W. M. \& Lehman, G. W. 1968 The shape of a sessile drop. J. Appl. Phys. 39, 1994-1996.

Rossen, W. R. 1990 a Theory of mobilization pressure gradient of flowing foams in porous media: I. Incompressible foam. J. Colloid and Interf. Sci. 136, 1-16.

Rossen, W. R. $1990 b$ Theory of mobilization pressure gradient of flowing foams in porous media: III. Asymmetric lamella shapes. J. Colloid and Interf. Sci. 136, 38-53.

Rossen, W. R. 1996 Foams in enhanced oil recovery. In Foams: Theory, Measurements and Applications (ed. R. K. Prud'homme \& S. A. Khan), Surfactant Science Series, chap. 2, pp. 99-187. New York: Marcel Dekker.

Rossen, W. R. 2013 Numerical challenges in foam simulation: A review (Paper SPE 166244). In SPE Annual Technical Conference and Exhibition, New Orleans, LA, 30th Sep.-2nd Oct..

Rossen, W. R. \& Bruining, J. 2007 Foam displacements with multiple steady states. SPE J. $12,5-18$.

Rossen, W. R., van Duijn, C. J., Nguyen, Q. P., Shen, C. \& Vikingstad, A. K. 2010 Injection strategies to overcome gravity segregation in simultaneous gas and water injection into homogeneous reservoirs. SPE J. 15, 76-90.

Rossen, W. R., Zellinger, S. C., Shi, J.-X. \& Lim, M. T. 1999 Simplified mechanistic simulation of foam processes in porous media. SPE J. 4, 279-287.

Saffman, P. G. \& TAYlor, G. 1958 The penetration of a fluid into a medium or Hele-Shaw cell containing a more viscous liquid. Proc. Roy. Soc. London Ser. A 245, 312-329.

SAhimi, M. 2011 Flow and Transport in Porous Media and Fractured Rock: From Classical Methods to Modern Approaches, 2nd edn. Weinheim, Germany: Wiley-VCH Publishers. 
Satomi, R., Grassia, P., Cox, S., Mishuris, G. \& Lue, L. 2013 Modelling a sheared semiinfinite film. Proc. Roy. Soc. London Ser. A 469, 20130359.

Saye, R. I. \& Sethian, J. A. 2011 The Voronoi implicit interface method for computing multiphase physics. Proc. Nat. Acad. Sci. USA 108, 19498-19503.

Schramm, L. L. \& Wassmuth, F. 1994 Foams: Basic principles. In Foams: Fundamentals and Applications in the Petroleum Industry (ed. L. L. Schramm), Advances in Chemistry, vol. 242, chap. 1, pp. 3-45. Washington, DC: American Chemical Society.

Sethian, J. A. 1996 A fast marching level set method for monotonically advancing fronts. Proc. Nat. Acad. Sci. USA 93, 1591-1595.

Sethian, J. A. 1999a Fast marching methods. SIAM Rev. 41, 199-235.

Sethian, J. A. $1999 b$ Level Set Methods and Fast Marching Methods: Evolving Interfaces in Computational Geometry, Fluid Mechanics, Computer Vision and Materials Science. Cambridge, New York: CUP.

Sethian, J. A. \& Popovici, A. M. 1999 3-d traveltime computation using the fast marching method. Geophys. 64, 516-523.

Sethian, J. A. \& Vladimirsky, A. 2000 Fast methods for the eikonal and related HamiltonJacobi equations on unstructured meshes. Proc. Nat. Acad. Sci. USA 97, 5699-5703.

Shan, D. \& Rossen, W. R. 2004 Optimal injection strategies for foam IOR. SPE J. 9, 132-150.

Smith, C. S. 1952 Grain shapes and other metallurgical applications of topology. In Metal Interfaces, pp. 65-108. American Society for Metals, Cleveland, Ohio.

TABeling, P., Zocchi, G. \& Libchaber, A. 1987 An experimental study of the Saffman-Taylor instability. J. Fluid Mech. 177, 67-82.

TAYLOR, J. E. 1995 The motion of multiple-phase junctions under prescribed phase-boundary velocities. J. Differ. Equations 119, 109-136.

Thome, H., Rabaud, M., Hakim, V. \& Couder, Y. 1989 The Saffman-Taylor instability: From the linear to the circular geometry. Phys. Fluids A 1, 224-240.

de Velde Harsenhorst, R. M., Dharma, A. S., Andrianov, A. \& Rossen, W. R. 2014 Extension and verification of a simple model for vertical sweep in foam SAG displacements. Accepted for publication by SPE Reservoir Evaluation 83 Engg (Paper SPE 164891).

Weaire, D. \& Hutzler, S. 1999 The Physics of Foams. Oxford: Clarendon Press.

Weaire, D. \& Kermode, J. P. 1983 Computer simulation of a 2-d soap froth. I. Method and motivation. Phil. Mag. B 48, 245-259.

Weaire, D. \& Kermode, J. P. 1984 Computer simulation of a 2-d soap froth. II. Analysis of results. Phil. Mag. B 50, 379-395.

Weaire, D. \& McMurry, S. 1996 Some fundamentals of grain growth. Solid State Phys. 50, $1-36$.

Xu, Q. \& Rossen, W. R. 2003 Effective viscosity of foam in periodically constricted tubes. Colloids and Surf. A, Physicochem. and Engg Aspects 216, 175-194.

Xu, Q. \& Rossen, W. R. 2004 Experimental study of gas injection in a surfactant-alternatinggas foam process. SPE Reservoir Evaluation \&3 Engg 7, 438-448.

Yan, W., Miller, C. A. \& Hirasaki, G. J. 2006 Foam sweep in fractures for enhanced oil recovery. Colloids and Surf. A, Physicochem. and Engg Aspects 282, 348-359.

Zanganeh, M. N., Kam, S. I., LaForce, T. C. \& Rossen, W. R. 2011 The method of characteristics applied to oil displacement by foam. SPE J. 16, 8-23.

Zanganeh, M. N. \& Rossen, W. R. 2013 Optimization of foam enhanced oil recovery: Balancing sweep and injectivity. SPE Reservoir Evaluation 85 Engg 16, 51-59.

Zhou, Z. H. \& Rossen, W. R. 1995 Applying fractional-flow theory to foam processes at the 'limiting capillary pressure'. SPE Advanced Technology Series 3, 154-162. 


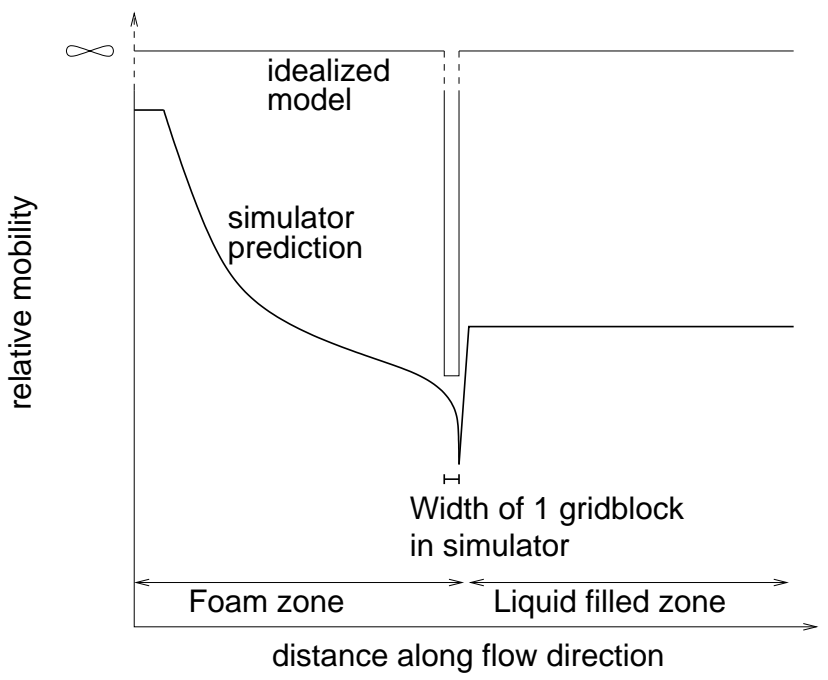

FIGURE 1. Typical plot of relative mobility (as defined in equation (2.1)) vs position as would be obtained (Shan \& Rossen 2004) from a simulation of a SAG flood in a reservoir, using a conventional reservoir engineering simulator (thick curve). The single grid block with extraordinarily low mobility at the leading edge of the foam zone is a spurious artifact of the simulator (Rossen 2013); otherwise the profile agrees well with fractional-flow theory predictions. The plot is not drawn to scale, but in the data of Shan \& Rossen (2004), relative mobility at the leading edge of the foam is an order of magnitude lower than that in the liquid, and two orders of magnitude less than that of the dry foam further back. Also shown is the idealised model (thin line) proposed by Shan \& Rossen (2004) and de Velde Harsenhorst et al. (2014), where the mobility is finite at the foam front only, but infinite everywhere else. 
(a)

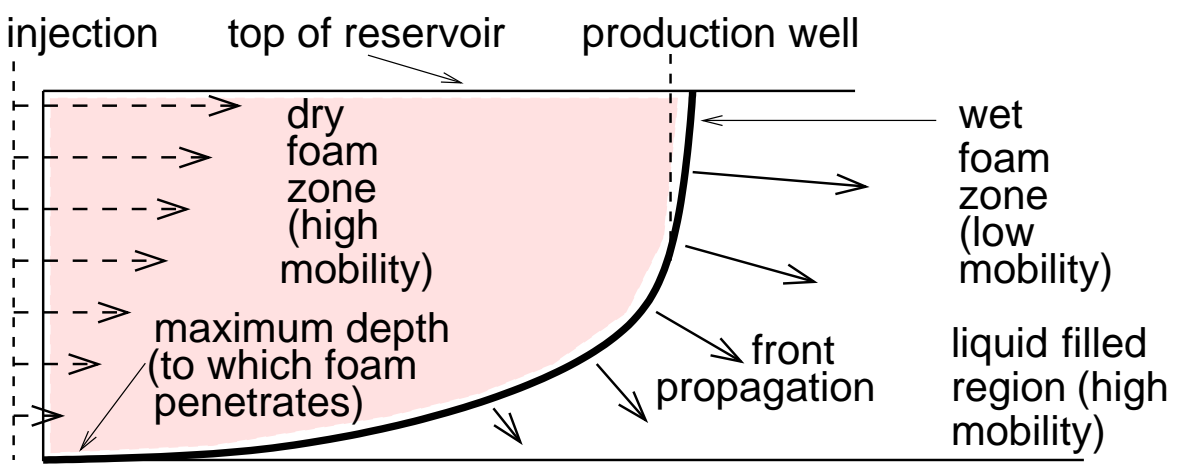

(b)

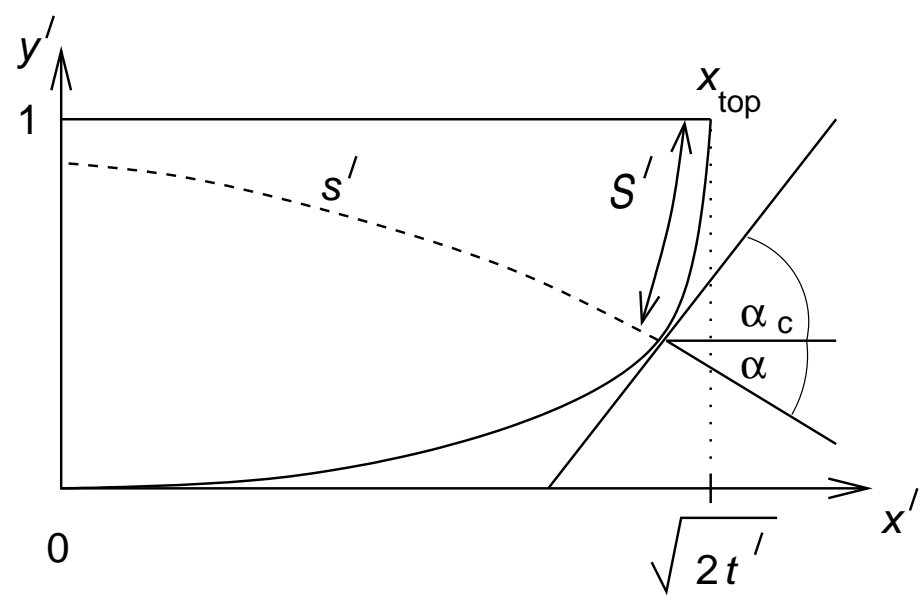

Figure 2. Definition sketch for the Shan \& Rossen (2004) and de Velde Harsenhorst et al. (2014) model. (a) As a result of injection of liquid followed by gas into a reservoir, foam penetrates into a liquid-filled region, and ultimately arrives at a production well. The foam is wettest and least mobile close to the foam front, and is drier and more mobile further back (since the bubbles are larger there). In the idealised model (Shan \& Rossen 2004; de Velde Harsenhorst et al. 2014), all the resistance to flow is concentrated at the foam front. Additionally there is a maximum depth to which foam can penetrate, which is set by the injection pressure and the gas-to-liquid density difference. Moreover the foam front meets the top of the reservoir at right angles. The foam front propagates along the direction of its local normal, at a speed that depends on depth. (b) Coordinate system and definitions of variables for computing the foam front. Coordinates have been made dimensionless such that the foam front covers the domain $0 \leqslant y^{\prime} \leqslant 1$, and is described by a function $y^{\prime}$ vs $x^{\prime}$. The normal to the front is an angle $\alpha$ from the horizontal, whilst $\alpha_{c}$ is the complement of $\alpha$. The arc length measured along the front (downwards from the top) is $S^{\prime}$, whilst the length measured along the path that material points have travelled to reach $\left(x^{\prime}, y^{\prime}\right)$ (starting from an original position somewhere on the $y^{\prime}$ axis) is $s^{\prime}$. The path travelled in the horizontal by the topmost point $x_{t o p}$ on the front is $\sqrt{2 t^{\prime}}$ (where $t^{\prime}$ is dimensionless time). 


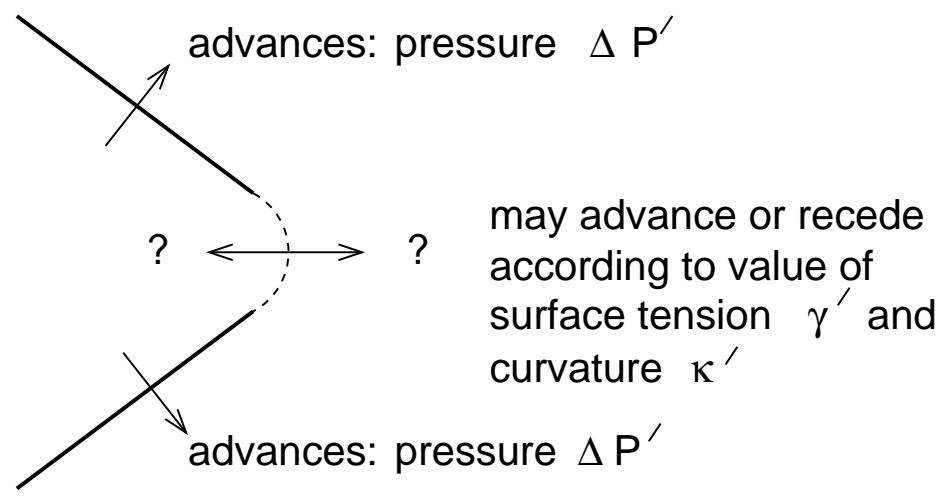

Figure 3. A hypothetical front shape for analysing the viscous froth model in the limit of weak surface tension. The straight sections of front advance (according to the driving pressure $\Delta P^{\prime}$ ), but whether the sharply curved section advances or recedes depends on the product of the surface tension $\gamma^{\prime}$ and the curvature $\kappa^{\prime}$ (compared to $\Delta P^{\prime}$ ). Specifically the curved section advances when $\gamma^{\prime} \kappa^{\prime}<\Delta P^{\prime}$ and recedes when $\gamma^{\prime} \kappa^{\prime}>\Delta P^{\prime}$. 
(a)

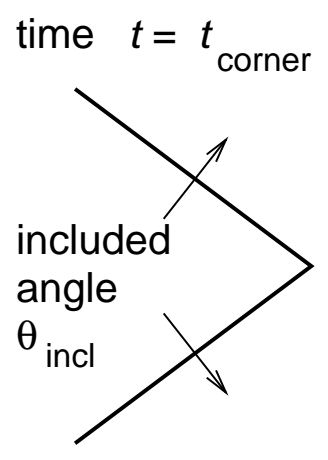

at later time

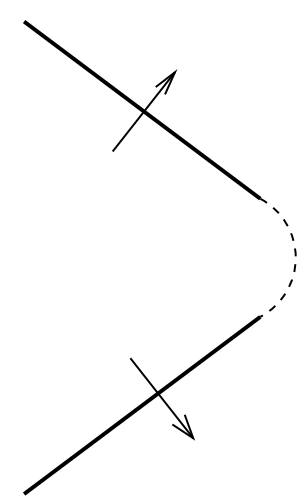

(b)

time $t=t$ arc

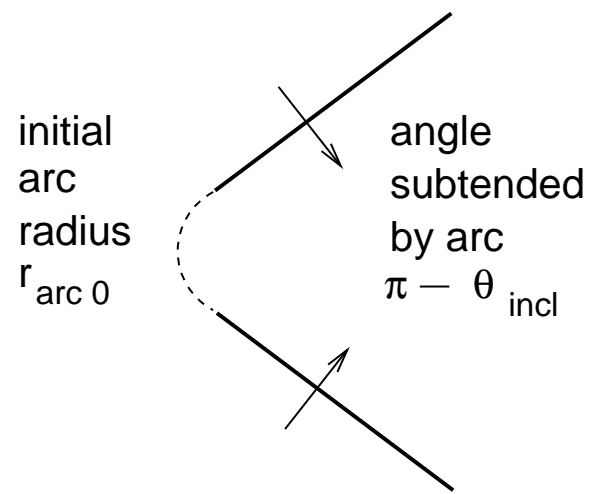

at later time

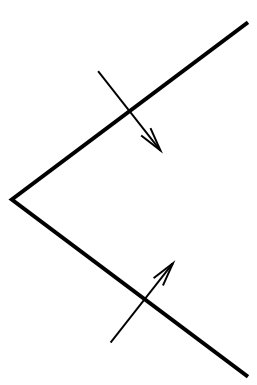

FiguRE 4. The evolution of certain front shapes according to the pressure-driven growth model. (a) Convex shape: an initial corner grows into a finite curvature arc, (b) Concave shape: an initially curved region focusses down to a sharp corner. 

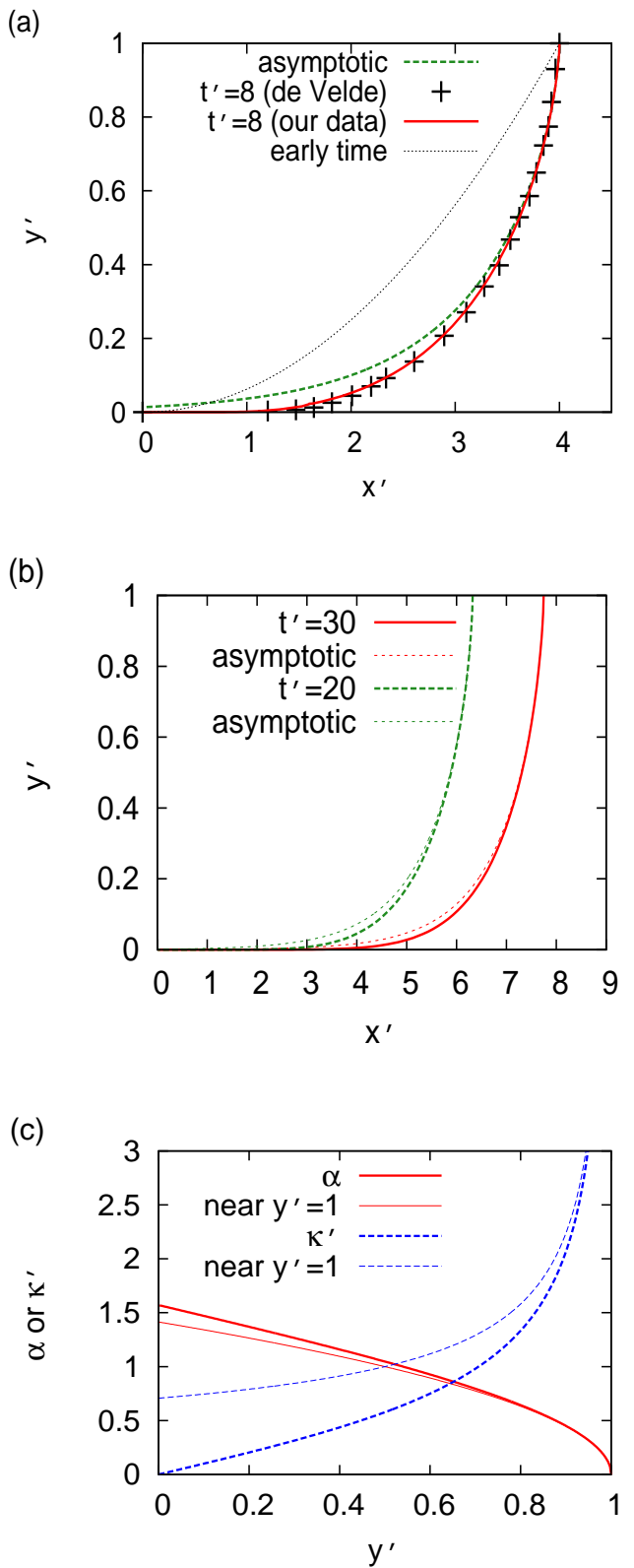

Figure 5. (a) Long-time asymptotic front shape (given by equation (4.9)) compared to finite time $t^{\prime}=8$ data taken from de Velde Harsenhorst et al. (2014). Here asymptotic data have been shifted to match the de Velde Harsenhorst et al. (2014) data at the leading edge at the top of the front. As an independent check, we have also recomputed the finite time $t^{\prime}=8$ data (verifying the findings of de Velde Harsenhorst et al. (2014)). In addition for comparison, we have plotted an early time solution of de Velde Harsenhorst et al. (2014) (which does not fit the $t^{\prime}=8$ data at all well). (b) Numerical front shapes $y^{\prime}$ vs $x^{\prime}$ plotted at times $t^{\prime}=20$ and $t^{\prime}=30$, and compared to the asymptotic long-time limit. Note that, whereas formula (4.9) gives $y^{\prime}$ vs $\xi$ (with $\xi=x^{\prime}-\sqrt{2 t^{\prime}}$ ), to avoid crowding on the figure, we have plotted here $y^{\prime}$ vs $x^{\prime}$ instead of $y^{\prime}$ vs $\xi$, thus separating data corresponding to different $t^{\prime}$. (c) Formulae for the angle $\alpha$ (between the front normal and the horizontal) and the front curvature $\kappa^{\prime}$ vs vertical coordinate $y^{\prime}$. These have been obtained via the long-time asymptotic solution (4.9), and limiting forms valid near the top of the reservoir $y^{\prime} \rightarrow 1$ have also been plotted (obtained via equations (4.13)-(4.14)). 
(a)
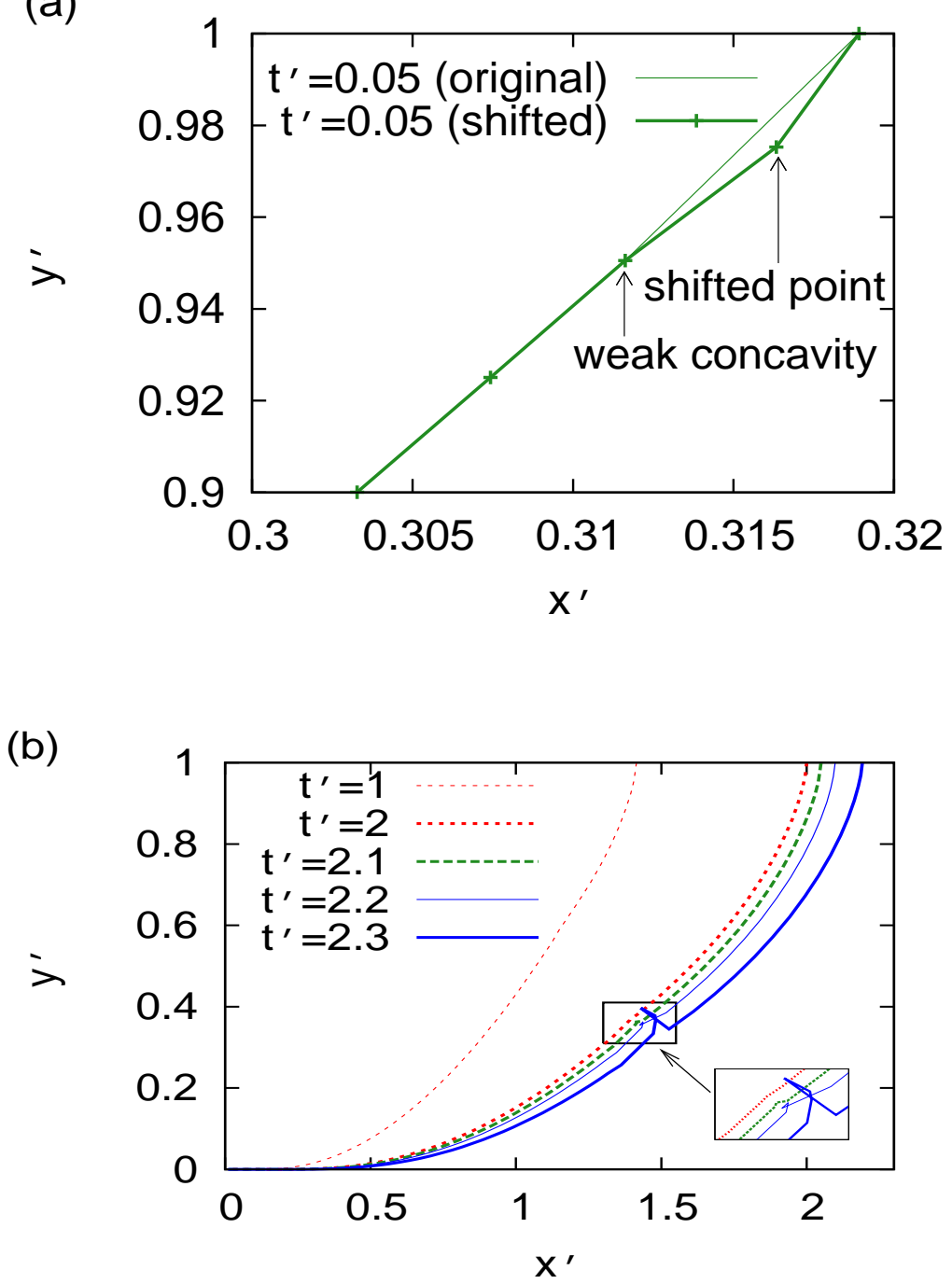

Figure 6. Numerical front shapes $y^{\prime}$ vs $x^{\prime}$ for the case of pressure-driven growth, where (a) at time $t^{\prime}=0.05$ we deliberately shift a data point that is very near the top boundary (note the scale for $x^{\prime}$ and $y^{\prime}$ ) a small distance (roughly 0.001 units) to the right of its correct horizontal position, introducing a weak concavity for the data point immediately below this. We then plot (b), over the full depth of the system, the subsequent front shape at time $t^{\prime}=1$, and also at times $t^{\prime}=2,2.1,2.2$ and 2.3 where the front is seen to evolve into a kink, although this occurs at quite some distance below the point where the concavity was originally introduced. 


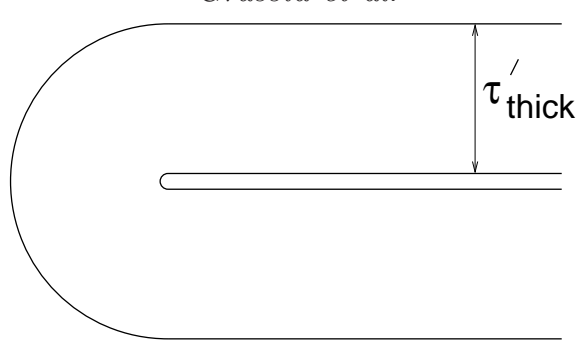

FIGURE 7. An object of thickness $\tau_{\text {thick }}^{\prime}$ cannot be bent into a curvature radius, measured along the outer edge, of less than $\tau_{\text {thick }}^{\prime}$. 


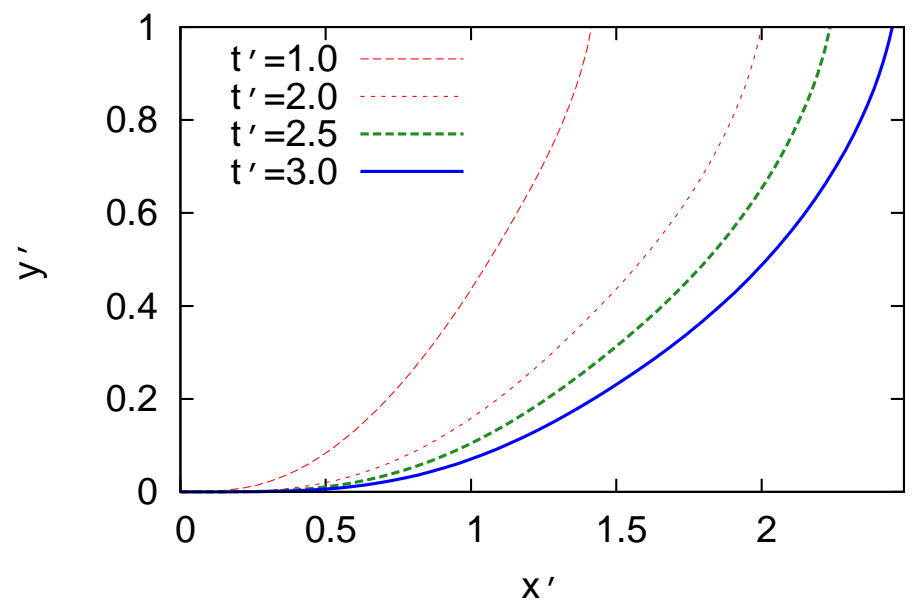

FiguRE 8. Numerical front shapes where (analogously to Figure 6) we deliberately move (at time $\left.t^{\prime}=0.0352\right)$ a data point near the top boundary a horizontal distance 0.001 away from its correct position, producing a concave defect on the front. Plots of $y^{\prime}$ vs $x^{\prime}$ are shown for times $t^{\prime}=1, t^{\prime}=2, t^{\prime}=2.5$ and $\left.t^{\prime}=3\right)$. These data are obtained via the viscous froth model equation (5.2) with $\tau=0.01$ - so (unlike the pressure-driven growth case of Figure 6) the defect heals and the front shape does not evolve towards a kink. 
56

(a)

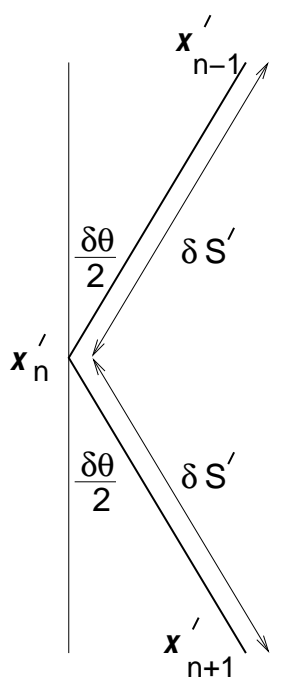

(b)

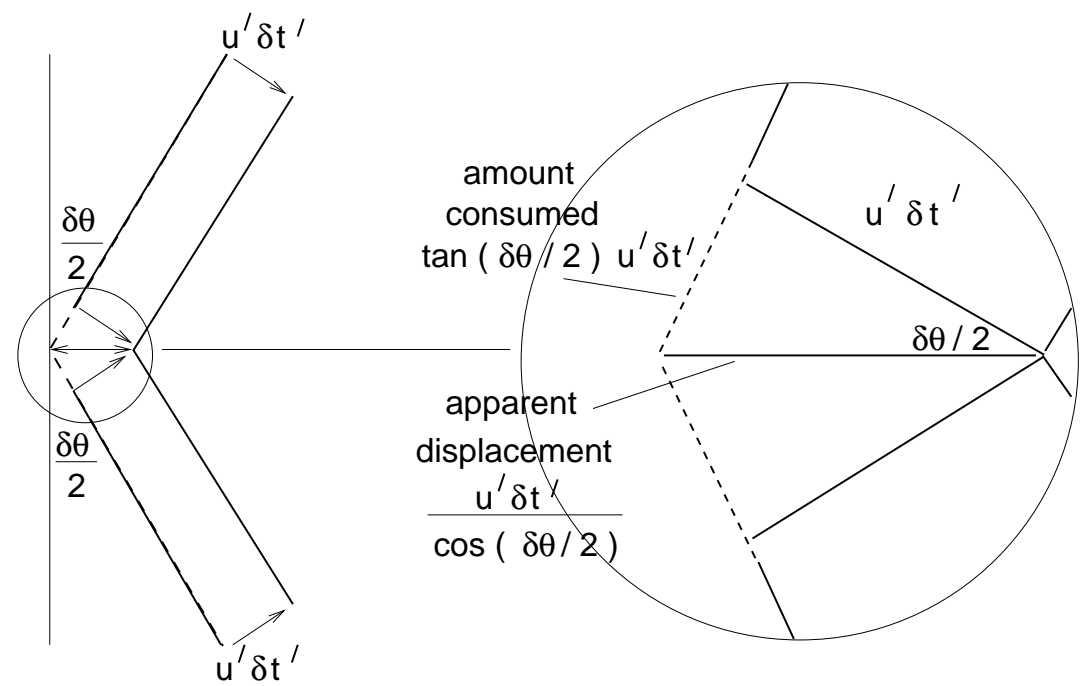

Figure 9. (a) Three points $\boldsymbol{x}_{n-1}^{\prime}, \boldsymbol{x}_{n}^{\prime}$ and $\boldsymbol{x}_{n+1}^{\prime}$ forming two adjacent segments on a concave front turning through an angle $\delta \theta$ over a distance $\delta S^{\prime}$ (measured from the midpoint of one segment to the next). (b) If segments propagate at speed $u^{\prime}$, then (over a time $\delta t$ ) an amount $u^{\prime} \delta t^{\prime} \tan \frac{\delta \theta}{2}$ of front is consumed from each segment. The intersection between the two segments appears to propagate by an amount $u^{\prime} \delta t^{\prime} / \cos \frac{\delta \theta}{2}$ and hence has apparent speed $u^{\prime} / \cos \frac{\delta \theta}{2}$. 


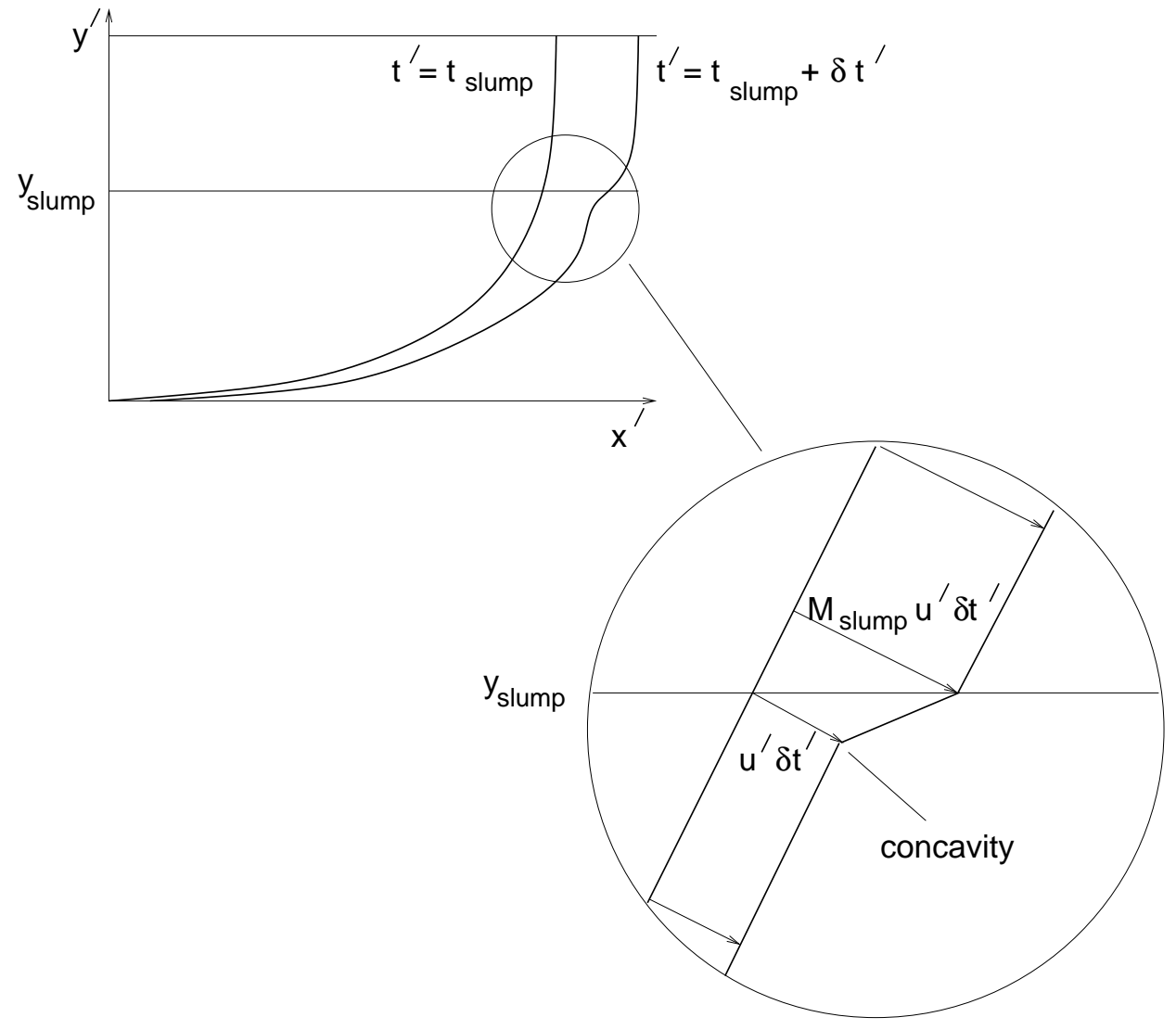

Figure 10. The case of liquid and/or surfactant slumping. After some time $t_{\text {slump }}$ material above the point $y_{\text {slump }}$ has an increased mobility (by a factor $M_{\text {slump }}$ ). Over time, the front may develop a significant concavity (compare the front shapes at time $t^{\prime}=t_{\text {slump }}$ with those a short time later at time $\left.t^{\prime}=t_{\text {slump }}+\delta t^{\prime}\right)$. The inset zooms in on the neighbourhood of location $y_{\text {slump }}$ near time $t_{\text {slump }}$. If material is moving at velocity $u^{\prime}$ immediately prior to slumping, then a time $\delta t^{\prime}$ later, material below $y_{\text {slump }}$ has displaced by $u^{\prime} \delta t^{\prime}$, but material above $y_{\text {slump }}$ has displaced more i.e. by $M_{\text {slump }} u^{\prime} \delta t^{\prime}$. A concavity is therefore seen to develop. 


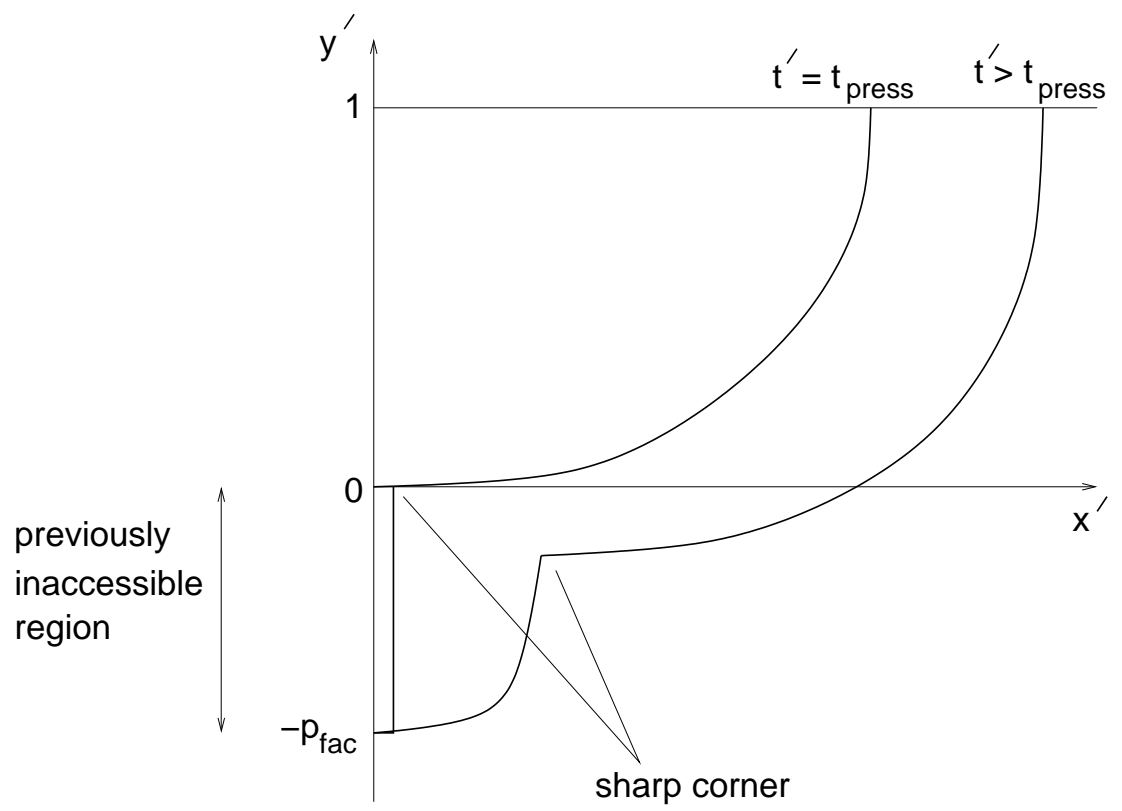

Figure 11. The case of an increase in driving pressure by a factor $1+p_{\text {fac }}$ realised at time $t^{\prime}=t_{\text {press }}$. Foam will invade previously inaccessible regions of the system (down to a depth $-p_{\text {fac }}$ immediately below the injection point) producing a sharp corner, which needs to be tracked for times $t^{\prime}>t_{\text {press }}$. 


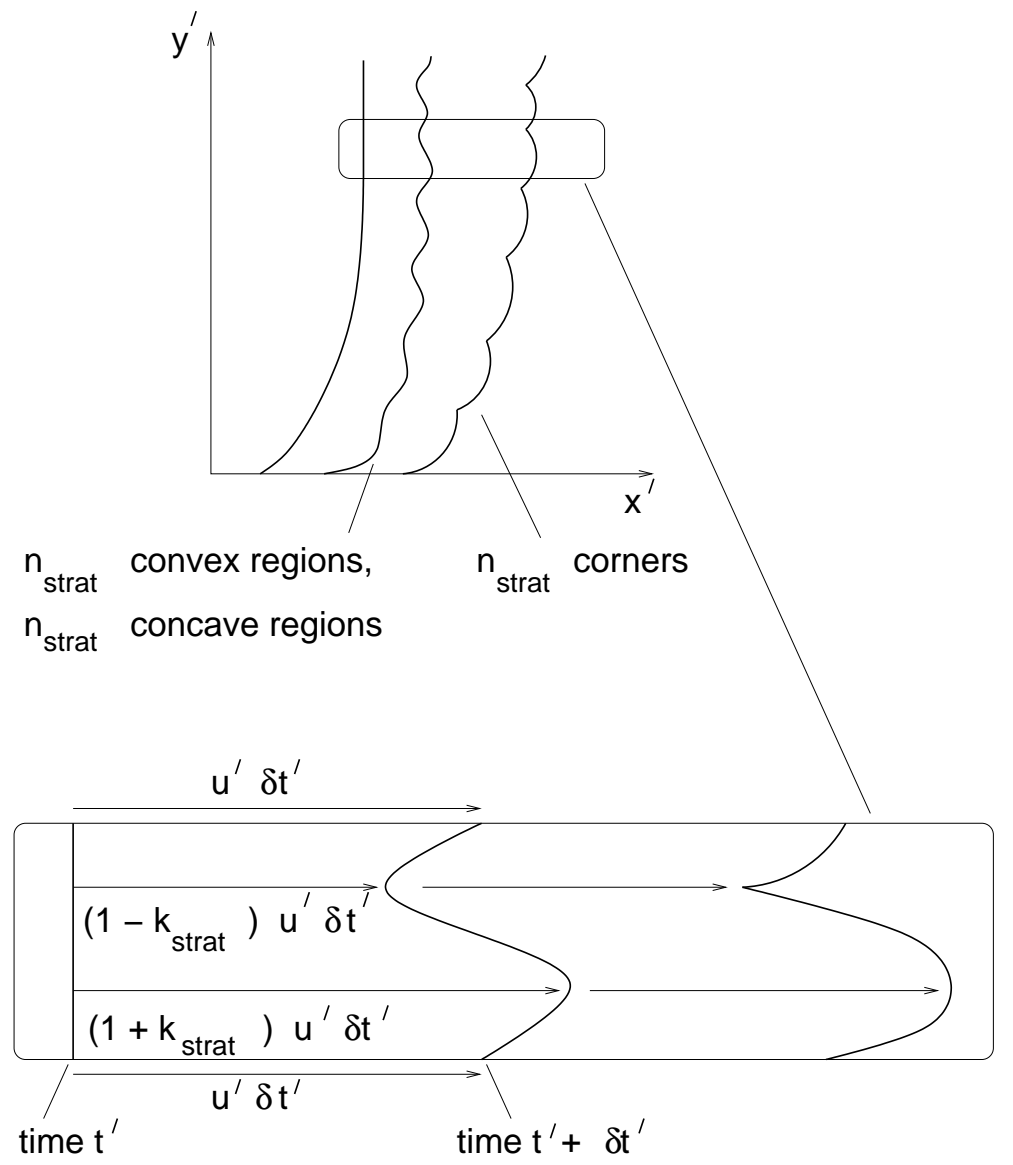

Figure 12. A foam front propagating over time in a heterogeneous system, with a sinusoidally modulated permeability, stratified into $n_{\text {strat }}$ lower permeability layers (with minimum permeability $1-k_{\text {strat }}$ ) interspersed with $n_{\text {strat }}$ higher permeability layers (with maximum permeability $\left.1+k_{\text {strat }}\right)$. The zoomed inset shows how an initially planar front propagating at average speed $u^{\prime}$ (and with maximal and minimal speed respectively $1 \pm k_{\text {strat }}$ times the average) develops, over a time step $\delta t^{\prime}$, concave and convex sections owing to the modulation; later on in time, the concave sections can focus into sharp corners. 


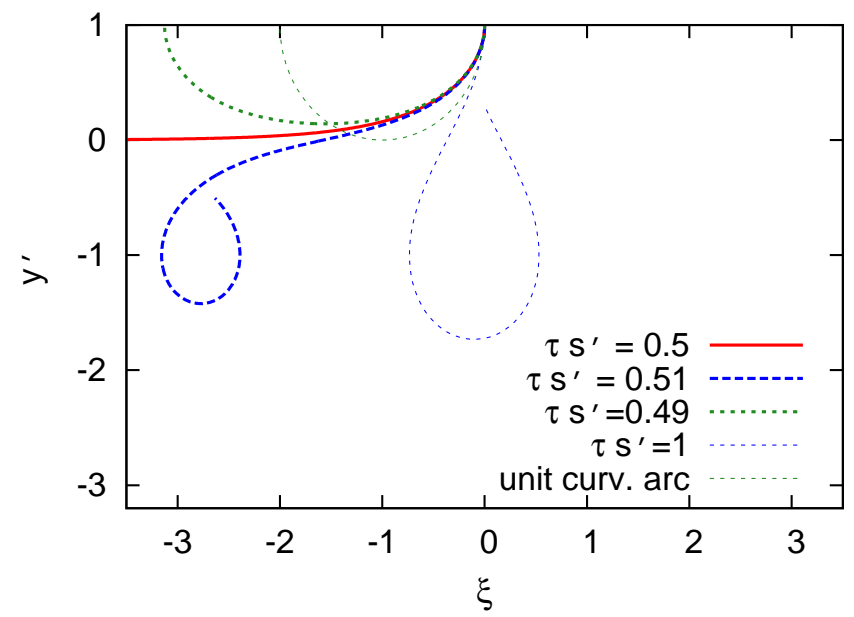

FiguRE 13. Solutions of the Young-Laplace equation (A 3) for various values of the parameter $\tau s^{\prime}$ where driving pressure difference $\Delta P^{\prime}$ is given by equation (2.11). Data are expressed in the form $\xi$ vs $y^{\prime}$, where $y^{\prime}$ denotes vertical coordinate and $\xi$ denotes horizontal coordinate (relative to the front location at the top of the reservoir). Curvature varies along the foam fronts as per a sessile or pendant drop. The solution we seek has $\tau s^{\prime}=\frac{1}{2}$ and has $y^{\prime} \rightarrow 0$ and $\xi \rightarrow-\infty$. For comparison, an arc of uniform unit curvature is also shown. 


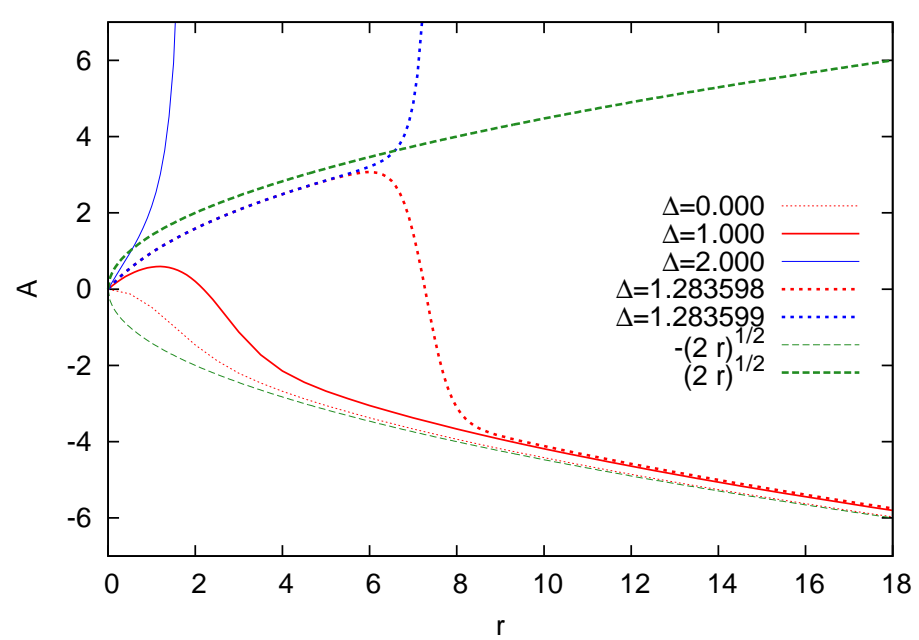

Figure 14. Solutions of equation (A 29) for $A$ vs $r$ various values of the parameter $\Delta$. Too large values of $\Delta$ lead to $A$ diverging to infinity. Too small values of $\Delta$ lead to $A$ approaching a negative solution branch $A \sim-\sqrt{2 r}$. Only for the correct value of $\Delta$ (between 1.283598 and 1.283599) does the solution follow close to the desired solution branch $A \sim \sqrt{2 r}$ for any significant distance. 


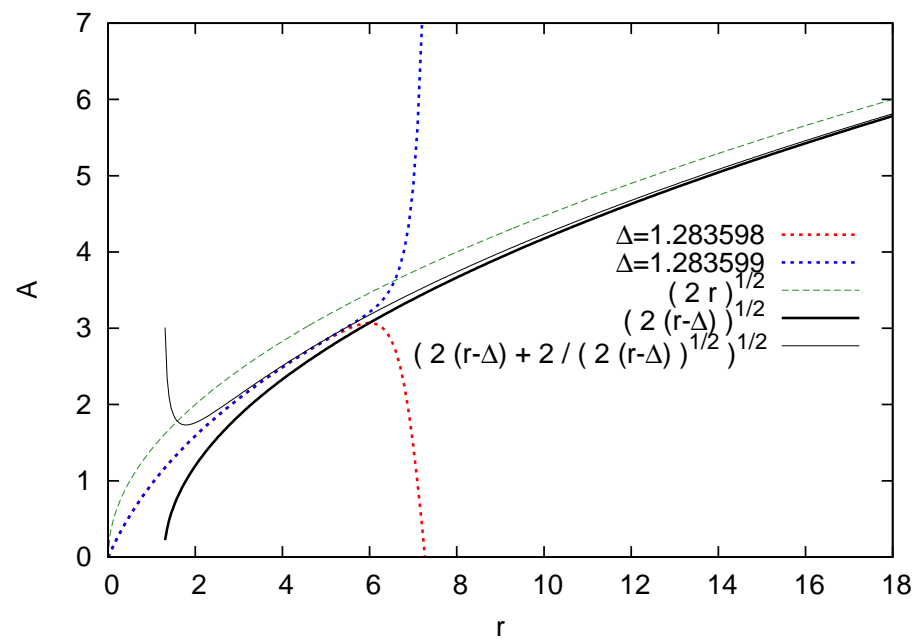

Figure 15. Numerical solutions of equation (A 29) for $A$ vs $r$ for the values of $\Delta$ either 1.283598 and 1.283599 (the two cases being virtually indistinguishable up to about $r \sim 6$ ), compared with the approximate solutions $A \sim \sqrt{2 r}, \sqrt{2(r-\Delta)}$ and $(2(r-\Delta)+2 / \sqrt{2(r-\Delta)})^{1 / 2}$. 


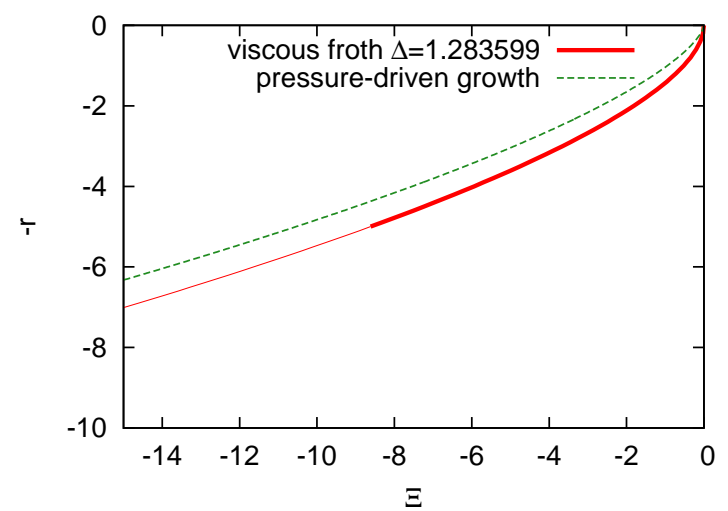

FiguRE 16. Solutions for front shape near the top of a reservoir, expressed in terms of the rescaled variables $-r$ vs $\Xi$ (rescaled vertical and horizontal variables respectively). The solution for the viscous froth model was obtained via equation (A 34) with the integrand $A$ in that equation either given by the numerical solution of equation (A 29) (thicker section of curve) or via the asymptotic form equation (A 31)(thinner section of curve). In each case the correctly chosen value of $\Delta$ (i.e. $\Delta=1.283599$ ) was used. The viscous froth solution is compared with the prediction of pressure-driven growth equation (A 35). 
(a)

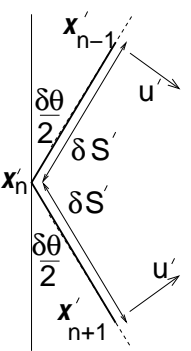

(b)

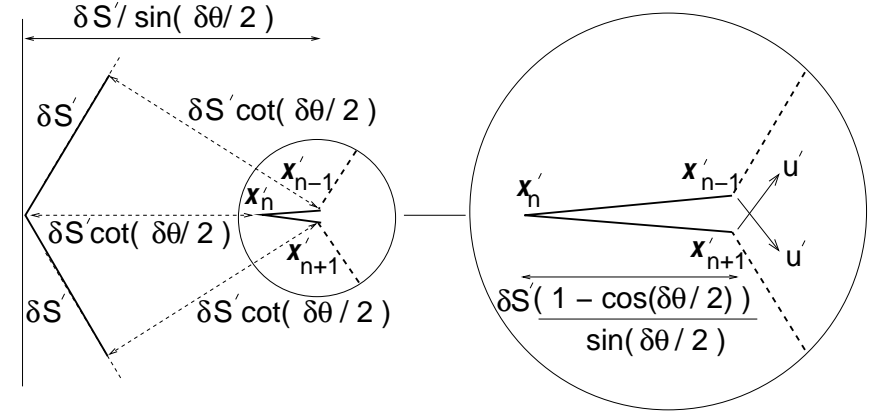

(c)

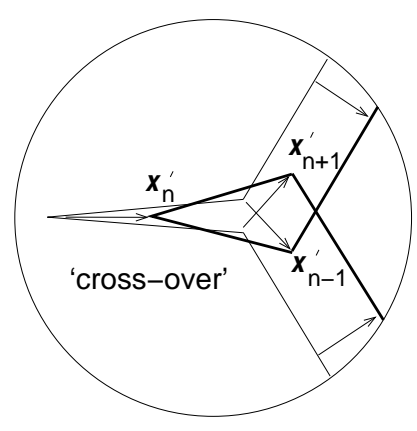

(d)

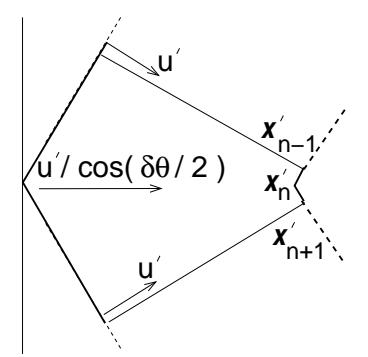

Figure 17. (a) Three discrete points on a foam front $\left(\boldsymbol{x}_{n-1}^{\prime}, \boldsymbol{x}_{n}^{\prime}\right.$ and $\left.\boldsymbol{x}_{n+1}^{\prime}\right)$ separated by a distance $\delta S^{\prime}$, and with the front turning through a total angle $\delta \theta$. The front is assumed to extend beyond $\boldsymbol{x}_{n-1}^{\prime}$ and $\boldsymbol{x}_{n+1}^{\prime}$ whilst remaining straight. Moreover material points propagate at speed $u^{\prime}$. (b) The new positions of the points $\boldsymbol{x}_{n-1}^{\prime}, \boldsymbol{x}_{n}^{\prime}$ and $\boldsymbol{x}_{n+1}^{\prime}$ (treated in the first instance as material points) a time $\left(\delta S^{\prime} \cot (\delta \theta / 2)\right) / u^{\prime}$ later. Points have displaced by $\delta S^{\prime} \cot (\delta \theta / 2)$. Points $\boldsymbol{x}_{n-1}^{\prime}$ and $\boldsymbol{x}_{n+1}^{\prime}$ are now coincident (and connect with dashed sections of front with the original orientation), but point $\boldsymbol{x}_{n}^{\prime}$ lags behind (by an amount $\delta S^{\prime}(1-\cos (\delta \theta / 2)) / \sin (\delta \theta / 2)$ as shown in the zoomed inset). Any further propagation at speed $u^{\prime}$ then leads, as in the zoomed view (c), to points $\boldsymbol{x}_{n-1}^{\prime}$ and $\boldsymbol{x}_{n+1}^{\prime}$ crossing over one another leading to the appearance of a spurious loop (compare the state shown by thick lines with the previous state which is shown here by thin lines). (d) Spurious behaviour can be avoided by propagating point $\boldsymbol{x}_{n}^{\prime}$ not as a material point with speed $u^{\prime}$, but rather as a geometric corner with speed $u^{\prime} / \cos (\delta \theta / 2)$. Point $\boldsymbol{x}_{n}^{\prime}$ then catches up with points $\boldsymbol{x}_{n-1}^{\prime}$ and $\boldsymbol{x}_{n+1}^{\prime}$ just as they become coincident. 